\title{
Mixed-symmetry fields in de Sitter space: a group theoretical glance
}

\author{
Thomas Basile, ${ }^{a, b}$ Xavier Bekaert ${ }^{a, c}$ and Nicolas Boulanger ${ }^{b, 1}$ \\ ${ }^{a}$ Laboratoire de Mathématiques et Physique Théorique, Unité Mixte de Recherche 7350 du CNRS, \\ Fédération de Recherche 2964 Denis Poisson, Université François Rabelais, \\ Parc de Grandmont, 37200 Tours, France \\ ${ }^{b}$ Groupe de Mécanique et Gravitation, Service de Physique Théorique et Mathématique, \\ Université de Mons - UMONS, \\ 20 Place du Parc, 7000 Mons, Belgique \\ ${ }^{c}$ B.W. Lee Center for Fields, Gravity and Strings, Institute for Basic Science, \\ Daejeon, South Korea \\ E-mail: thomas.basile@umons.ac.be, xavier.bekaert@lmpt.univ-tours.fr, \\ nicolas. boulanger@umons.ac. be
}

ABSTRACT: We derive the characters of all unitary irreducible representations of the $(d+1)$ dimensional de Sitter spacetime isometry algebra $\mathfrak{s o}(1, d+1)$, and propose a dictionary between those representations and massive or (partially) massless fields on de Sitter spacetime. We propose a way of taking the flat limit of representations in (anti-) de Sitter spaces in terms of these characters, and conjecture the spectrum resulting from taking the flat limit of mixed-symmetry fields in de Sitter spacetime. We identify the equivalent of the scalar singleton for the de Sitter (dS) spacetime.

Keywords: Field Theories in Higher Dimensions, Gauge Symmetry

ArXIV EPRINT: 1612.08166

\footnotetext{
${ }^{1}$ Research Associate of the Fund for Scientific Research — FNRS (Belgium).
} 


\section{Contents}

1 Introduction $\quad 2$

$2 \mathfrak{s o}(1, d+1)$ unitary irreducible representations $\quad 4$

2.1 Classification 4

2.2 Structure and characters of the corresponding modules 5

2.2.1 Principal series 7

$\begin{array}{lll}2.2 .2 & \text { Complementary series } & 8\end{array}$

2.2.3 Exceptional series 8

$\begin{array}{lll}2.2 .4 & \text { Discrete series } & 13\end{array}$

2.3 Masslessness: AdS vs dS 14

3 Flat limit $\quad \mathbf{1 5}$

3.1 Anti-de Sitter case: the Brink-Metsaev-Vasiliev mechanism 16

$\begin{array}{ll}3.2 & \text { Principal and complementary series } \\ \end{array}$

$\begin{array}{lll}3.3 & \text { Exceptional series } & 19\end{array}$

3.3.1 Even spacetime dimensions 20

$\begin{array}{lll}3.3 .2 & \text { Odd spacetime dimensions } & 22\end{array}$

$\begin{array}{lll}3.4 & \text { Discrete series } & 26\end{array}$

$\begin{array}{lll}4 & \text { Conclusions } & 27\end{array}$

$\begin{array}{ll}\text { A Massive scalar field on (anti) de Sitter spacetime } & 29\end{array}$

B Classification of $\mathfrak{s o}(2, d)$ unitary irreducible representations \& their characters $\quad 30$

C Verma interlude $\quad 32$

C.1 Verma module 32

C.2 Generalised Verma module 33

D Characters from Bernstein-Gel'fand-Gel'fand resolutions 34

$\begin{array}{ll}\text { E Poincaré characters revisited } & 40\end{array}$

E.1 Massive representations 40

E.2 Massless representations 42

F Branching rules for $\mathfrak{s o}(d) \quad 43$

F.1 $\mathfrak{s o}(2 r+1) \downarrow \mathfrak{s o}(2 r) \quad 44$

F.2 $\mathfrak{s o}(2 r) \downarrow \mathfrak{s o}(2 r-1) \quad 45$ 
G.1 Anti-de Sitter case 48

$\begin{array}{ll}\text { G.2 de Sitter case } & 49\end{array}$

\section{Introduction}

The importance of mixed-symmetry fields (i.e. fields whose physical components carry representations of the little group described by Young diagrams of height greater than one) is no longer to be emphasised: whether motivated by string theory — where they make up most of the spectrum - or, more generically, by quantum field theory in arbitrary dimensions - where they are the central objects of interest in the sense that they are the most general fields one may consider. ${ }^{1}$ At the free level, equations of motions for massless mixed-symmetry fields in flat spacetime were spelled out by Labastida $[1,2]^{2}$ (see $[4,5]$ for a proof that his equations and trace constraints describe the right propagating degrees of freedom and [6] for the fermionic case), and later given in the unfolded form [7]. In anti-de Sitter $\left(\operatorname{AdS}_{d+1}\right)$ spacetime, the study of massless mixed-symmetry fields was mostly driven by Metsaev [8-10] who gave both the group theoretical description of the corresponding $\mathfrak{s o}(2, d)$ module and (partially) gauged fixed equations, similar to Fronsdal's equation for totally symmetric fields [11] in the De Donder gauge, i.e. the action of the wave operator on the field is equal to a critical mass square, together with divergencelessness and tracelessness conditions, and completed by similar equations on the gauge parameters. Again, those equations were later revisited using the unfolded approach in $[12,13]$ for the unitary cases and in $[14,15]$ for the non-unitary cases, thereby generalizing the LopatinVasiliev equations [16] that describe the propagation of free massless, totally-symmetric fields around (A)dS spacetime. The generalised Bargmann-Wigner equations for arbitrary mixed-symmetry (partially)-massless gauge fields, both unitary and non-unitary, were given in $[12,13]$ in a framework where both spacetime signatures are treated on the same footing. Notice that the presentation of the equations of motion for massless fields in the form of a Fierz-Pauli system was given by Metsaev [8-10] for the AdS signature. This being said, the only difference with the dS signature, as far as the form of the Fierz-Pauli-like equations is concerned, resides in the sign of the eigenvalue of the Laplace-Beltrami operator in the wave equation for the mixed-symmetry gauge potential. However, a nontrivial difference between the positive and negative cosmological constant cases is the question of unitarity of the fields and their corresponding (irreducible) representations, which is one of the main issues investigated in the present paper.

In deriving all these equations, the constraints imposed by gauge symmetry were crucial. At the group theoretical level, the presence of this symmetry in $\operatorname{AdS}_{d+1}$ translates into

\footnotetext{
${ }^{1}$ They anyway appear upon electric-magnetic duality transformation of fields of spin two (or higher) in spacetime dimensions greater than four.

${ }^{2}$ See also [3] for an earlier, non minimal formulation (starting from the light-cone gauge).
} 
the fact that the representation corresponding to the gauge field is constructed as a quotient: the gauge parameter module forms a submodule to be modded out from the gauge field module in order to obtain an irreducible representation (irrep) of the isometry algebra $\mathfrak{s o}(2, d)$. Unitary and irreducible representations (UIRs) of $\mathfrak{s o}(2, d)$ are well known by now, and the correspondence with fields in $\mathrm{AdS}_{d+1}$ is also well established, in the physically important cases of bounded energy. However, a similar dictionary between $(d+1)$-dimensional de Sitter spacetime $\left(\mathrm{dS}_{d+1}\right)$ fields and the UIRs of its isometry algebra, $\mathfrak{s o}(1, d+1)$, is still missing in full generality. A first step in this direction was made in $[17,18]$ where the authors studied UIRs of the de Sitter group corresponding to massive and massless scalars. Arbitrary spin, and especially mixed-symmetry massless fields remain elusive in this respect. In the present paper, we fill this gap and relate arbitrary mixed-symmetry fields in de Sitter spacetime to UIRs of $\mathfrak{s o}(1, d+1)$ given in the mathematical $[19,20]$ and Euclidean Conformal Field Theory (CFT) literature [21, 22].

On top of that, mixed-symmetry gauge fields in $\mathrm{AdS}_{d+1}$ were shown to have quite an interesting flat limit [23]: starting from a gauge field in $\mathrm{AdS}_{d+1}$ with symmetry encoded by an arbitrary $\mathfrak{s o}(d)$ Young diagram $\mathbb{Y}$ and sending the cosmological constant $\Lambda$ to zero yields a spectrum of massless fields in flat spacetime composed of all possible fields labelled by $\mathfrak{s o}(d-1)$ Young diagrams obtained from $\mathbb{Y}$ by removing boxes in each of the last rows of each block (until it reaches the length of the row just below), leaving the first (upper) block untouched. For proofs of this spectrum, see [12, 13, 24]. This property can be reformulated as the group theoretical statement that a massless, mixed-symmetry, irrep of $\mathfrak{s o}(2, d)$ contracts to a direct sum of massless Poincaré irreps, the spectrum of massless fields on Minkowski spacetime being given by a truncation of the branching of $\mathbb{Y}$ with respect to $\mathfrak{s o}(d-1) \subset \mathfrak{s o}(d)$. We show that a similar situation occurs in $\mathrm{dS}_{d+1}$, the difference being that the spectrum is given by a truncated branching of $\mathbb{Y}$ where the last block (i.e. the lowest one) is left untouched in the unitary case. In light of the recent revival of interest for higher-spin theories formulated around flat spacetime [25-29], such a mechanism relating massless fields of arbitrary spin in either $\mathrm{AdS}_{d+1}$ or $\mathrm{dS}_{d+1}$ (which are more natural backgrounds for higherspin gravity) to their flat spacetime counter parts can be of great help in understanding the subtleties of these flat spacetime formulations as limits of theories in curved spacetime.

This paper is organised as follows:

- In section 2 we expose the classification of the UIRs of $\mathfrak{s o}(1, d+1)$ that can be found in the literature,

- In section 3 we use the previously derived character formulae to investigate the flat limit of (massive and) massless field/representations of $\mathfrak{s o}(1, d+1)$,

- We conclude in section 4 with some considerations on the possibility of a singleton type representation for $\mathfrak{s o}(1, d+1)$ and a corresponding Flato-Fronsdal theorem,

- Finally, we include a few technicalities in several appendices. 


\section{$2 \mathfrak{s o}(1, d+1)$ unitary irreducible representations}

We begin this section by reviewing the classification of the UIRs of $\mathfrak{s o}(1, d+1)$ and spelling out their characters (derived in appendix D). With the latter at hand, we try to establish a dictionary between these UIRs and massive or massless fields in de Sitter space.

The Lie algebra $\mathfrak{s o}(1, d+1)$ is spanned by antisymmetric and Hermitian generators $M_{A B}=-M_{B A},\left(M_{A B}\right)^{\dagger}=M_{A B},(A, B=0,1, \ldots, d, d+1)$ subject to the commutation relations:

$$
\left[M_{A B}, M_{C D}\right]=i\left(\eta_{B C} M_{A D}+\eta_{A D} M_{B C}-\eta_{A C} M_{B D}-\eta_{B D} M_{A C}\right)
$$

with $\eta_{A B}=\operatorname{diag}(-1,+1, \ldots,+1)$. One can perform the following redefinitions:

$$
D:=-i M_{0 d+1}, P_{i}:=M_{0 i}+M_{d+1 i}, K_{i}:=M_{0 i}-M_{d+1 i}, \quad(i=1,2, \cdots, d),
$$

thereby leading to the commutation relations for the conformal algebra of the $d$-dimensional Euclidean space: ${ }^{3}$

$$
\begin{cases}{\left[M_{i j}, M_{k l}\right]=i \delta_{j k} M_{i l}+\ldots,} & {\left[K_{i}, P_{j}\right]=2\left(i M_{i j}+\delta_{i j} D\right),} \\ {\left[M_{i j}, P_{k}\right]=2 i \delta_{k[j} P_{i]},} & {\left[M_{j k}, K_{i}\right]=2 i \delta_{i[j} K_{k]},} \\ {\left[D, P_{i}\right]=P_{i},} & {\left[D, K_{i}\right]=-K_{i} .}\end{cases}
$$

In this interpretation, the subalgebra $\mathfrak{s o}(d)=\operatorname{span}\left\{M_{i j}\right\}$ corresponds to infinitesimal rotations of the Euclidean space $\mathbb{R}^{d}$. Let $r:=\left[\frac{d}{2}\right]$ denote the rank of $\mathfrak{s o}(d)$ (with $[x]$ denoting the integer part of $x$ ). The remaining generators $D, P_{i}$ and $K_{j}$ correspond respectively to infinitesimal dilations, translations and special conformal transformations of the Euclidean space $\mathbb{R}^{d}$.

\subsection{Classification}

Let us start by recalling the classification of the generalised Lorentz (or de Sitter) group UIRs, established in $[21,22]$ (see also [19, 20, 30, 31]). As was originally shown by HarishChandra, for non-compact semisimple Lie groups these representations can be classified in different series called "principal", "complementary" and "discrete" (see [32, 33] for more details). As in the more familiar case $\mathfrak{s o}(2, d)$ (see appendix B for a summary of the relevant irreps of $\mathfrak{s o}(2, d))$, each highest-weight irrep of $\mathfrak{s o}(1, d+1)$ is labelled by an $\mathfrak{s o}(d)$ highestweight $\vec{s}=\left(s_{1}, \ldots, s_{r}\right)$ corresponding to the spin (where the entries $s_{i} \in \frac{1}{2} \mathbb{N},{ }^{4}$ are such that $s_{1} \geqslant s_{2} \geqslant \cdots \geqslant s_{r}$ and $\left.^{5} 2 s_{1}=\cdots=2 s_{r} \bmod 2\right)$ and an additional $\mathfrak{s o}(1,1)$ weight $\Delta_{c} \in \mathbb{C}$ corresponding to the "conformal weight" of the representation. ${ }^{6}$ The Young diagram $\mathbb{Y}$ has rows of lengths $\left[s_{i}\right]$ (with $i=1,2, \cdots, r$ ). For tensorial representations, the entries of $\vec{s}$

\footnotetext{
${ }^{3}$ The above generators of $\mathfrak{s o}(1, d+1)$ are related to those of [21] by $X_{i j}=i M_{i j}, C_{i}=K_{i}, T_{j}=P_{j}$.

${ }^{4}$ Strictly speaking, for $d=2 r$ the last entry $s_{r}$ can be negative as well, and the irreps where the last two entries only differ by a sign are related by a discrete transformation. For this reason, this subtlety will be ignored in this subsection but taken into account later on.

${ }^{5}$ In other words, the components of the $\mathfrak{s o}(d)$ highest-weight are either all integer or all half-integer.

${ }^{6}$ Notice that the conformal weight $\Delta$ is always a real number in the case of UIRs of $\mathfrak{s o}(2, d)$, as the corresponding Hermitian generator (the energy) spans $\mathfrak{s o}(2)$.
} 
are integers, thus $\left[s_{i}\right]=s_{i}$ for bosonic fields. In order to have a simpler uniform treatment (including the fermionic case), with a slight abuse of notation the lenghts of the rows of the Young diagram corresponding to a (tensor)-spinor representation of $\mathfrak{s o}(d)$ with half-integer entries will nevertheless be denoted $s_{i}$, as in the bosonic case (although strictly speaking they are equal to $\left.\left[s_{i}\right]=s_{i}-\frac{1}{2}\right)$. The list of UIRs of $\mathfrak{s o}(1, d+1)$ is as follows:

- Principal series: $\Delta_{c}=\frac{d}{2}+i \rho$, with $\rho \in \mathbb{R}$ and $\vec{s}$ arbitrary.

- Complementary series: $s_{i}=0$ for $p+1 \leqslant i \leqslant\left[\frac{d-1}{2}\right]$, where $p \in\{0,1,2, \cdots, r-1\}$ is the number of nonvanishing entries in $\vec{s}$ (thus, when $d$ is even, at least one entry vanishes) $\Delta_{c}=\frac{d}{2}+c$ with $c \in \mathbb{R}$ such that $0<|c|<\frac{d}{2}-p$.

- Exceptional series: $s_{i}=0$ for $p+1 \leqslant i \leqslant r$ where $p \in\{1,2, \cdots, r\}$ and $\Delta_{c}=d-p$ or $\Delta_{c}=p$. They are essentially the boundary points of the complementary series.

- Discrete series (only for $d=2 r+1$ ): $\Delta_{c}=\frac{d}{2}+k$ with $k \in \frac{1}{2} \mathbb{N}$ and $0<k \leqslant s_{r}$ (thus all entries in $\vec{s}$ are non-vanishing).

Notice that an irrep labelled by $\left[\Delta_{c} ; \vec{s}\right]$ is (partially) equivalent to the irrep labelled by $\left[d-\Delta_{c} ; \vec{s}\right]$ [34]. In Euclidean CFT literature, the representation for $\left[d-\Delta_{c} ; \vec{s}\right]$ is usually referred to as the "shadow" of the one for $\left[\Delta_{c} ; \vec{s}\right]$.

Remark. The existence of a whole series of UIRs, the discrete one, only in even spacetime dimensions (i.e. odd $d$ ) can seem a bit strange at first sight, but it can actually be explained by a standard result due to Harish-Chandra. Indeed, he proved that a real semisimple Lie group possesses a discrete series of UIRs if and only if it has a compact Cartan subgroup. In the case of $\mathrm{SO}(1, d+1)$ of interest for us, which is of rank $r+1$, the maximal compact subgroup is $\mathrm{SO}(d+1)$, which has rank $\left[\frac{d+1}{2}\right]$. In even spacetime dimensions (i.e. $d=2 r+1$ ), the group $\mathrm{SO}(1, d+1)$ has the same rank $r+1$ as its maximal compact subgroup $\mathrm{SO}(d+1)$ and therefore has a compact Cartan subgroup, namely the one of the subgroup $\mathrm{SO}(d+1)$. In odd spacetime dimensions (i.e. $d=2 r)$ however, the rank of $\mathrm{SO}(d+1)$ is $r$ and does not match that of $\mathrm{SO}(1, d+1)$, which means that there is no compact Cartan subgroup, hence the absence of a discrete series for $d=2 r$.

\subsection{Structure and characters of the corresponding modules}

The above listed UIRs were constructed and classified using the method of induced representations (see [22], Chap. IV, appendix B), a construction that we will briefly outline for the sake of completeness.

First of all, we need to introduce a few subalgebras of $\mathfrak{g}=\mathfrak{s o}(1, d+1)$ (and the corresponding subgroups of $G=\mathrm{SO}(1, d+1))$ :

- $\mathfrak{K}=\mathfrak{s o}(d+1)$ is its maximal compact subalgebra;

- $\mathfrak{a}=\mathfrak{s o}(1,1)=\operatorname{span}\{D\}$ is the abelian subalgebra generated by the dilation operator;

- $\mathfrak{m}=\mathfrak{s o}(d)=\operatorname{span}\left\{M_{i j}\right\}$ is the centraliser of $\mathfrak{a}$ in $\mathfrak{K}$, generated by the $d$-dimensional rotations; 
- $\mathfrak{n}=\mathbb{R}^{d}=\operatorname{span}\left\{K_{i}\right\}$ is the nilpotent (and abelian) subalgebra generated by the special conformal transformations.

Starting with the Iwasawa decomposition (i.e. the decomposition of a semisimple Lie algebra into its maximal compact subalgebra, an abelian and a nilpotent subalgebra) of $\mathfrak{g}$, one can introduce the corresponding Iwasawa decomposition at the group level $G=K A N$, with $K, A$ and $N$ the Lie subgroups of which $\mathfrak{K}, \mathfrak{a}$ and $\mathfrak{n}$ are respectively the Lie algebras. One can further introduce the centraliser $M \equiv \mathrm{SO}(d)$ of $A$ in $K$, and define the parabolic subgroup $P=M A N$ of $\mathrm{SO}(1, d+1)$ in terms of its Langlands decomposition (the product of semisimple, abelian, and nilpotent subgroups). This might be better understood at the algebra level, where a parabolic subalgebra $\mathfrak{p}$ of some semisimple Lie algebra $\mathfrak{g}$ is defined as any subalgebra containing the Borel subalgebra $\mathfrak{b}$ of $\mathfrak{g}$, the latter being the subalgebra made out of the Cartan subalgebra together with the subalgebra generated by the raising (or lowering) operators (or equivalently the subalgebra dual to the space of positive, or negative, roots). In our case, the Cartan subalgebra of $\mathfrak{g}=\mathfrak{s o}(1, d+1)$ that we will consider is composed of the Cartan subalgebra of $\mathfrak{m}=\mathfrak{s o}(d)$ and $\mathfrak{a}=\mathfrak{s o}(1,1)$. The parabolic subalgebra we are interested in here is $\mathfrak{p}=\mathfrak{s o}(1,1) \notin \mathfrak{i s o}(d):=\operatorname{span}\left\{M_{j k}, K_{i}, D\right\}$, with $\mathfrak{i s o}(d)=\mathfrak{s o}(d) \notin \mathfrak{n}:=\operatorname{span}\left\{M_{j k}, K_{i}\right\}$.

Secondly, consider a finite-dimensional UIR $\left(\mathbb{V}_{\boldsymbol{\lambda}}, \rho_{\boldsymbol{\lambda}}\right)$ of the corresponding parabolic subgroup $P$. It is labelled by the weight $\boldsymbol{\lambda}=\left[\Delta_{c} ; \vec{s}\right]$, since a standard lemma (cf. Lemma 1 in section 19 of [35]) ensures that the nilpotent subgroup $N$ acts trivially in such a case. This irrep induces a representation $\left(\mathscr{C}\left(G, \mathbb{V}_{\boldsymbol{\lambda}}\right), \mathcal{R}_{\boldsymbol{\lambda}}\right)$ of $G$ on the space $\mathscr{C}\left(G, \mathbb{V}_{\boldsymbol{\lambda}}\right)$ of functions on the group $G$ with value in $\mathbb{V}_{\boldsymbol{\lambda}}$ and subject to the covariance condition:

$$
f(g x)=\rho_{\boldsymbol{\lambda}}\left(x^{-1}\right) f(g), \quad \forall f \in \mathscr{C}\left(G, \mathbb{V}_{\boldsymbol{\lambda}}\right), g \in G, x \in P,
$$

via:

$$
\left(\mathcal{R}_{\boldsymbol{\lambda}}(g) f\right)\left(g^{\prime}\right)=f\left(g^{-1} g^{\prime}\right), \quad \forall f \in \mathscr{C}\left(G, \mathbb{V}_{\boldsymbol{\lambda}}\right), g, g^{\prime} \in G .
$$

Following [21], these induced representations, where one uses the action of the group on itself, will be called the elementary representations. The "subrepresentation theorem" (see e.g. [21], p.47) supports this terminology: every UIR of $\mathrm{SO}(1, d+1)$ is (infinitesimally) equivalent to an irreducible component of an elementary representation.

In order to classify the UIRs of $\mathrm{SO}(1, d+1)$, one thus has to decompose the elementary representation into its irreducible and unitary components, which gives rise to the above mentioned series of representations. ${ }^{7}$ The principal series corresponds to a continuum of UIRs of $G$ that are induced by a UIRs of $P$ in which the nilpotent part $N$ is represented trivially, and are already irreducible as constructed above. The discrete series corresponds to, as their name suggests, a discrete set of UIRs induced by $P$ and appearing in the decomposition of the elementary representation. As mentioned in the previous subsection,

\footnotetext{
${ }^{7}$ Note that the construction sketched here has no claim at providing an exhaustive picture of the theory of induced representations, nor at complete mathematical rigor. Our only purpose is to give an intuitive picture of the way the $\mathrm{SO}(1, d+1)$ UIRs discussed in this paper were classified and their relation with the corresponding algebra representations.
} 
the exceptional series, singled out in the classification of $\mathfrak{s o}(1, d+1)$ UIRs actually consists of irreps with a conformal weight $\Delta_{c}$ at the unitarity bound of the complementary series.

At the algebra level, this construction corresponds to generalised Verma modules, reviewed in more details in appendix C. At the level of Lie algebras, induced representations are constructed as follows:

- Given a Lie subalgebra $\mathfrak{h} \subset \mathfrak{g}$ and a finite-dimensional $\mathfrak{h}$-module $\mathbb{V}$, the module $\mathcal{U}(\mathfrak{g}) \otimes \mathcal{U}(\mathfrak{h}) \mathbb{V}($ where $\mathcal{U}(\mathfrak{g})$ is the universal enveloping algebra of $\mathfrak{g})$ makes up a representation of $\mathfrak{g}$;

- To see that, recall that an element $x$ of $\mathcal{U}(\mathfrak{g}) \otimes_{\mathcal{U}(\mathfrak{h})} \mathbb{V}$ reads $\left(y_{1} \ldots y_{k}\right) \otimes v$ with $y_{1}, \ldots, y_{k} \in \mathfrak{g}$ and $v \in \mathbb{V}$, hence there exists a natural action of $\mathfrak{g}$ on this module, namely $\rho(z) x:=\left(z y_{1} \ldots y_{k}\right) \otimes v$ for $z \in \mathfrak{g}$, induced by the associative product in the universal enveloping algebra;

- Finally, the subscript $\mathcal{U}(\mathfrak{h})$ on the tensor product symbol simply means that $\forall x \in$ $\mathcal{U}(\mathfrak{h}), \quad \rho(x)(\mathbb{1}) \otimes v=(x) \otimes v=(\mathbb{1}) \otimes(\tilde{\rho}(x) v)$ where $\tilde{\rho}$ is the representation of $\mathcal{U}(\mathfrak{h})$ on $\mathbb{V}$ (arising from the one of $\mathfrak{h}$ on $\mathbb{V}$ ).

Here we are interested in $\mathfrak{h}=\mathfrak{p}=\mathfrak{s o}(1,1) \notin \mathfrak{i s o}(d)$ and $\mathfrak{g}=\mathfrak{s o}(1, d+1)$. We will consider generalised Verma modules based on this algebra: $\mathcal{V}_{\boldsymbol{\lambda}}:=\mathcal{U}(\mathfrak{g}) \otimes_{\mathcal{U}(\mathfrak{p})} \mathbb{V}_{\boldsymbol{\lambda}}$, where as previously $\boldsymbol{\lambda}=\left[\Delta_{c} ; \vec{s}\right]$ is an $\mathfrak{s o}(1,1) \oplus \mathfrak{s o}(d)$ highest-weight and $\mathbb{V}_{\boldsymbol{\lambda}}$ the corresponding $\mathfrak{s o}(1,1) \oplus \mathfrak{s o}(d)$ highest-weight module. Using the Poincaré-Birkhoff-Witt theorem, the generalised Verma module $\mathcal{V}_{\boldsymbol{\lambda}}$ can be equivalently defined as: $\mathcal{V}_{\boldsymbol{\lambda}}=\mathcal{U}(\mathfrak{t}) \otimes \mathbb{V}_{\boldsymbol{\lambda}}$, as $\mathfrak{t}=\operatorname{span}\left\{P_{i}\right\}$ is the complement of $\mathfrak{p}$ in $\mathfrak{s o}(1, d+1)$. In other words, one can think of a generalised Verma module as the module obtained by acting with all the lowering operators of the algebra that do not belong to the chosen parabolic subalgebra (in our case, the translation generators) on a finitedimensional highest-weight space $\mathbb{V}_{\boldsymbol{\lambda}}$ of the parabolic subalgebra $\mathfrak{p}$ instead of a highestweight vector, as would be the case in the (more standard) context of Verma modules.

The character of a generalised Verma module $\mathcal{V}_{\left[\Delta_{c} ; \vec{s}\right]}$ reads:

$$
\chi_{\left[\Delta_{c} ; \vec{s}\right]}^{\mathrm{dS}}(q, \vec{x})=q^{\Delta_{c}} \chi_{\vec{s}}^{\mathfrak{s o}(d)}(\vec{x}) \mathcal{P}^{(d)}(q, \vec{x}),
$$

where the function $\mathcal{P}^{(d)}(q, \vec{x})$ is the character of the elementary representation of trivial weight $[0 ; \overrightarrow{0}]$ (i.e. an $\mathfrak{s o}(2, d)$ scalar function) and is given by:

$$
\mathcal{P}^{(d)}(q, \vec{x})=\prod_{i=1}^{r} \frac{1}{\left(1-q x_{i}\right)\left(1-q x_{i}^{-1}\right)} \times\left\{\begin{array}{cl}
1 & \text { if } d=2 r \\
\frac{1}{1-q} & \text { if } d=2 r+1
\end{array}\right.
$$

\subsubsection{Principal series}

The representations of the principal series are induced from irreps of $\mathfrak{p}$ with complex $\mathfrak{s o}(1,1)$ weight $\Delta_{c}=\frac{d}{2}+i \rho$, where $\rho \in \mathbb{R}$ and arbitrary $\mathfrak{s o}(d)$ highest-weight (i.e. arbitrary spin). The corresponding generalised Verma modules are irreducible as $\mathfrak{s o}(1, d+1)$-modules. The 
following character was derived originally in [36], where the author computed it working at the $\mathrm{SO}(1, d+1)$ group level:

$$
\chi_{\left[\Delta_{c} ; \vec{s}\right]}^{\mathrm{dS}}(q, \vec{x})=\left(q^{\frac{d}{2}+i \rho} \chi_{\vec{s}_{+}}^{\mathfrak{s o}(d)}(\vec{x})+q^{\frac{d}{2}-i \rho} \chi_{\vec{s}_{-}}^{\mathfrak{s o}(d)}(\vec{x})\right) \mathcal{P}^{(d)}(q, \vec{x}),
$$

where $\vec{s}_{ \pm}$denotes "chiral" pairs of $\mathfrak{s o}(d)$ highest-weights (when the distinction is relevant), i.e.

$$
\vec{s}_{ \pm}=\left(s_{1}, \ldots, s_{r-1}, \pm s_{r}\right) \quad \text { for } d=2 r
$$

and

$$
\vec{s}_{+}=\vec{s}_{-}=\vec{s} \quad \text { for } d=2 r+1 .
$$

Notice that, as a consequence of working at the group level, both chiralities (accompanied with a conjugation of the $\mathfrak{s o}(1,1)$ weight) appear in the above expression. The principal series of representations is known to describe massive fields in $\mathrm{dS}_{d+1}$ (see for instance [17, 37]) which complies with the fact that their definition does not involve any quotient of elementary representations and therefore do not exhibit any gauge invariance.

\subsubsection{Complementary series}

The elementary representations of the complementary series are also irreducible from the start, and as such their $\mathrm{SO}(1, d+1)$ characters [36] read:

$$
\chi_{\left[\Delta_{c} ; \vec{s}\right]}^{\mathrm{dS}}(q, \vec{x})=\left(q^{\frac{d}{2}+c} \chi_{\vec{s}_{+}}^{\mathfrak{s o}(d)}(\vec{x})+q^{\frac{d}{2}-c} \chi_{\vec{s}_{-}}^{\mathfrak{s o}(d)}(\vec{x})\right) \mathcal{P}^{(d)}(q, \vec{x}) .
$$

For the same reason as in the principal series, these UIRs should correspond to massive fields. One may phrase the difference between those two series of massive fields as follows: those in the principal series describe "very massive" fields whereas those in the complementary series correspond to "not-so-massive" fields. Let us expand a little bit: when writing down a wave equation for a field in (A)dS, one would refer to the eigenvalue $m^{2}$ of the Laplace-Beltrami operator as the mass squared of this field, for lack of a group-theoretical invariant concept as in Minkowski space where it is exactly the quadratic Casimir operator of the Poincaré group. However, this mass term is also related to the value of the quadratic Casimir operator for $\mathfrak{s o}(1, d+1)$ or $\mathfrak{s o}(2, d)$ and thereby it can be expressed in term of the conformal weight $\Delta_{c}$. Having at hand the relation between $\Delta_{c}, m$ and the spin of this field (encoded in the $\mathfrak{s o}(d)$ part of the Casimir operator), principal series fields have a higher corresponding mass squared $m^{2}$. This distinction is illustrated in the simple example of a massive scalar field, detailed in appendix A and sketched in figure 1.

\subsubsection{Exceptional series}

The representations of the exceptional series are those irreps induced by UIRs of $\mathfrak{p}$ with conformal weight at the unitary bound of the complementary series, i.e. $\Delta_{c}=d-p$ or $\Delta_{c}=p$, with $p$ the height of the Young diagram $\mathbb{Y}$ labeling the $\mathfrak{s o}(d)$ part of the irrep. As a consequence, the corresponding generalised Verma module contains null vectors, i.e. these elementary representations are reducible. One therefore has to find all submodules contained in the generalised Verma module constructed from the $\mathfrak{p}$-irrep $[d-p ; \vec{s}]$. This survey 


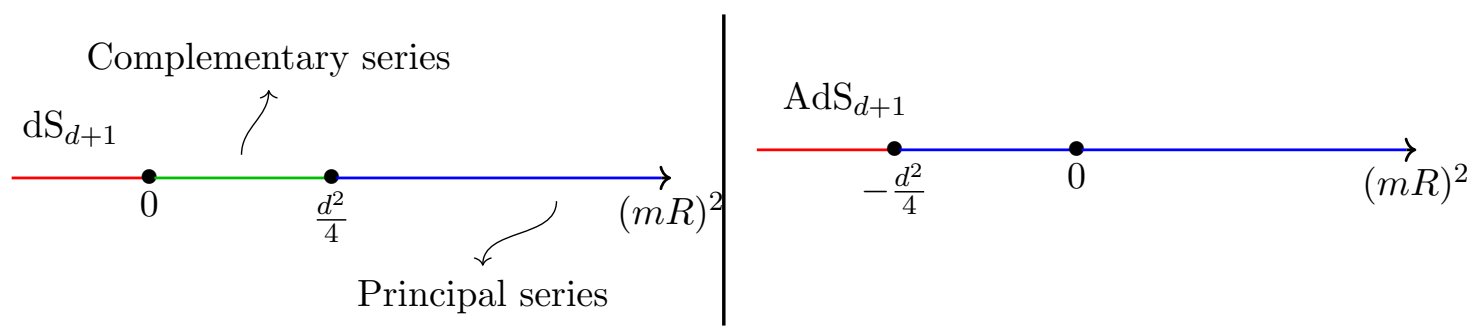

Figure 1. Unitary (blue and green), and non-unitary (red) regions for the squared mass of a scalar field in de Sitter (left) and anti-de Sitter space (right).

was done in $[31,38]$ at the group level. At the algebra level, one can rely on the so-called Bernstein-Gel'fand-Gel'fand (BGG) resolutions to perform the same analysis. The idea is the following: given a generalised Verma module $\mathcal{V}_{\boldsymbol{\lambda}}$, where $\boldsymbol{\lambda}=\left[\Delta_{c} ; \vec{s}\right]$ represents the highest-weight characterising the irrep of $\mathfrak{p}$ from which it is built, the BGG theorem gives a criterion for an element of the Weyl group of $\mathfrak{s o}(1, d+1)$ to yield a highest-weight defining a submodule, when applied to $\boldsymbol{\lambda}$. It furthermore provides a resolution of the irreducible module $\mathcal{D}_{\boldsymbol{\lambda}}$ in the form of an exact sequence involving $\mathcal{V}_{\boldsymbol{\lambda}}$ and its submodules. This analysis and the BGG resolution is known in the case of the complex algebra $\mathfrak{s o}^{*}(d+2)$ and was used in [39] to classify the possible systems of unfolded equations invariant under the conformal algebra $\mathfrak{s o}(2, d)$. Using these resolutions, one can derive the character of an irreducible representation in the exceptional series in terms of characters of $\mathfrak{p}$, as detailed in appendix D (see also the appendix $\mathrm{F}$ of [40] for an earlier derivation of such a dictionary for characters).

In order to be able to write the characters in a more compact way, we will use the following notation:

- $\mathbb{Y}_{p}$ will represent a Young diagram of height $p$ (with $p \leqslant r$ ), i.e.

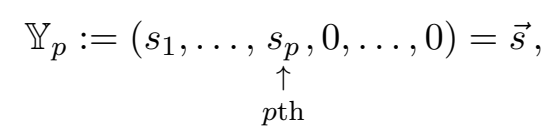

with $s_{p}>0$;

- $\left(\mathbb{Y}_{p}, \mathbf{1}^{m}\right)$ will represent a Young diagram of height $p+m$ obtained by adding $m$ rows of length one below $\mathbb{Y}_{p}$, i.e.

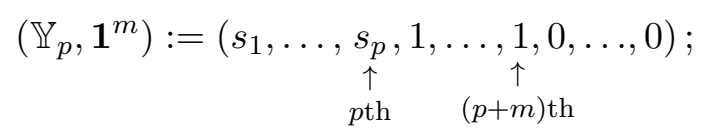

- $\breve{Y}_{p}^{(i)}$ will represent the diagram obtained from $\mathbb{Y}_{p}$ after having (i) removed its $i$ th row and (ii) removed one box in each of the row below the previously removed one, i.e.

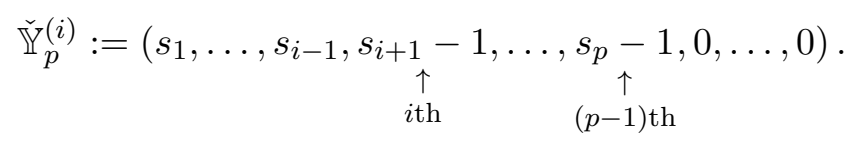

Depending on the parity of $d$, the structure of the irreducible module obtained from $\mathcal{V}_{\left[d-p ; \mathbb{Y}_{p}\right]}$ is slightly different, which is why we need to treat both cases separately. 
- Even spacetime dimension $(d=2 r+1)$ :

$$
\begin{aligned}
\chi_{\left[d-p ; \mathbb{Y}_{p}\right]}^{\mathrm{dS}}(q, \vec{x})= & \sum_{m=0}^{r-p}(-1)^{m}\left(q^{p+m}-q^{d-p-m}\right) \chi_{\left(\mathbb{Y}_{p}, \mathbf{1}^{m}\right)}^{\mathfrak{s o}(d)}(\vec{x}) \mathcal{P}^{(d)}(q, \vec{x}) \\
& -\sum_{\ell=1}^{p}(-1)^{p+1+\ell} q^{s_{\ell}+d-\ell} \chi_{\mathbb{Y}_{p}^{\mathfrak{s o}(\ell)}}^{\mathfrak{s}(d)}(\vec{x}) \mathcal{P}^{(d)}(q, \vec{x}) ;
\end{aligned}
$$

- Odd spacetime dimension $(d=2 r)$ :

$$
\begin{aligned}
\chi_{\left[d-p ; \mathbb{Y}_{p}\right]}^{\mathrm{dS}}(q, \vec{x})= & 2 \sum_{\ell=1}^{p}(-)^{p+\ell+1} q^{s_{\ell}+d-\ell} \chi_{\mathbb{Y}_{p}^{(\ell)}}^{\mathfrak{s o}(d)}(\vec{x}) \mathcal{P}^{(d)}(q, \vec{x}) \\
& +\sum_{n=0}^{r-p-1}(-)^{n}\left(q^{d-p-n}+q^{p+n}\right) \chi_{\left(\mathbb{Y}_{p}, \mathbf{1}^{n}\right)}^{\mathfrak{s o}(d)}(\vec{x}) \mathcal{P}^{(d)}(q, \vec{x}) \\
& \quad+(-)^{r-p} q^{d / 2}\left(\chi_{\left(\mathbb{Y}_{p}, \mathbf{1}_{+}^{r-p}\right)}^{\mathfrak{s o}(d)}(\vec{x})+\chi_{\left(\mathbb{Y}_{p}, \mathbf{1}_{-}^{r-p}\right)}^{\mathfrak{s o}(d)}(\vec{x})\right) \mathcal{P}^{(d)}(q, \vec{x}) .
\end{aligned}
$$

Remark. In even spacetime dimensions, the character (2.13) exactly reproduces the formula for the $\mathrm{SO}(1, d+1)$ character derived in [36], upon rewriting it in way that makes the $\mathfrak{s o}(d)$ part of the character explicit. However, in odd spacetime dimensions, (2.14) differs from the formula given in [36], namely the first line of our formula is not recovered from the expression of [36]. Nevertheless, we want to stress that we have derived the expression (2.14) as well as all the characters of the Lie algebra $\mathfrak{s o}(1, d+1)$ presented in this paper using the BGG resolutions recalled in appendix D.

Knowing the structure of the corresponding modules, we can now propose a field theoretical interpretation. First of all, the presence of submodules in the generalised Verma module $\mathcal{V}_{\left[\Delta_{c} ; \vec{s}\right]}$ suggests the presence of gauge invariance for the corresponding fields, i.e. the exceptional series UIRs should correspond to massless fields in $\mathrm{dS}_{d+1}$. However, the simplest massless fields that one could think of, which are the totally symmetric, spin- $s$ gauge fields, seem to be either absent of this series of irreps or do not have the expected conformal weight: being labelled by the single row Young diagram $\mathbb{Y}=(s, 0, \ldots, 0)$, the associated conformal weight in this series would be $\Delta_{c}=d-1$ and not the usual $s+d-2$. Our interpretation of this apparent contradiction is that the conformal weight and Young diagram characterising a UIR in the exceptional series actually corresponds to that of the curvature (see our definition below) of the massless field that it describes.

In order to discuss gauge field and curvatures for arbitrary Young diagrams, we will use the following notation:

- A Young diagram will be generically seen as composed of $B$ blocks, each of the them being of individual length $\ell_{I}$ and height $h_{I}(1 \leqslant I \leqslant B)$;

- We will write the cumulated height of the first $I$ blocks $p_{I}:=\sum_{J=1}^{I} h_{J}\left(\right.$ thus $p_{1}=h_{1}$ ), and hence the total height of the Young diagram is $p_{B}$ that we will denote $p$ hereafter; 
- Therefore, the Young diagram will be written as

$$
\vec{s}=(\underbrace{\ell_{1}, \ldots, \ell_{1}}_{h_{1}}, \underbrace{\ell_{2}, \ldots, \ell_{2}}_{h_{2}}, \ldots, \underbrace{\ell_{B}, \ldots, \ell_{B}}_{h_{B}}, 0, \ldots, 0) \equiv\left(\ell_{1}^{h_{1}}, \ell_{2}^{h_{2}}, \ldots, \ell_{B}^{h_{B}}\right)
$$

In the case of $B=3$ blocks:

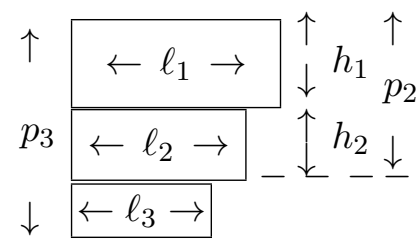

Recall that a massless gauge field $\varphi_{\mathbb{Y}}$ of mixed symmetry ${ }^{8}$ described by the Young diagram $\mathbb{Y}$ is subject to gauge transformations of the form:

$$
\delta_{\epsilon}^{(I)} \varphi_{\mathbb{Y}}=\nabla_{(I)} \epsilon_{\mathbb{Y}_{I}^{\prime}}+\text { traces }
$$

where $\mathbb{Y}_{I}^{\prime}$ is the Young diagram obtained from $\mathbb{Y}$ by removing one box in the last row of its $I$ th block, and $\nabla_{(I)}$ means that the derivative acting on $\epsilon$ is projected, in the sense that the resulting object has the symmetry of $\mathbb{Y}$. In this paper, what we call the curvature $^{9}$ is obtained by acting on $\varphi_{\mathbb{Y}}$ with as many derivatives as the length of the "activated" block, the $I$ th one, and projecting them so that the resulting object has the symmetry of $\mathbb{Y}$ to which one extra row was added to the activated block. The above described objects are illustrated in the figure 2 .

The previous discussion should be refined a little bit, taking into account partial masslessness, initially introduced in [41-44] for totally symmetric gauge fields (see also [45-49] for recent works). These fields are subject to higher derivative gauge transformations, and inherit their name from the fact that they propagate an intermediate number of degrees of freedom between those of a bona fide massless field and a massive one (the canonical example being that of a spin- $s$ partially massless field of depth $t$ in 4 dimensions, which propagates $2 t$ helicities, namely $\pm s, \pm(s-1), \ldots, \pm(s-t+1)$, with $t \in\{1,2, \cdots, s\})$. The generalization of partially massless fields to mixed-symmetries was considered in $[12,13]$. These fields are subject to gauge transformations of the form:

$$
\delta_{\epsilon}^{(I)} \varphi_{\mathbb{Y}}=\underbrace{\nabla \ldots \nabla}_{t} \epsilon_{\mathbb{Y}_{I}^{\prime}(t)}+\text { traces }
$$

\footnotetext{
${ }^{8}$ Recall that such a field corresponds to a Lorentz tensor whose spacetime indices have the symmetry properties of the $\mathfrak{s o}(1, d)$ Young diagram $\mathbb{Y}$ (i.e. it is completely traceless), and subject to a divergencelessness condition which ensures that the field only propagates the degrees of freedom corresponding to the little group representation, i.e. the $\mathfrak{s o}(d)$ Young diagram $\mathbb{Y}$.

${ }^{9}$ The "primary Weyl tensor" $[12,13]$ is obtained by acting with $\ell_{I}-\ell_{I+1}$ derivatives on the gauge field $\varphi_{\mathbb{Y}}$ and by projecting the resulting object on the symmetries of the $\mathfrak{s o}(1, d)$ Young diagram obtained by adding $\ell_{I}-\ell_{I+1}$ boxes to $\mathbb{Y}$ in the $\left(p_{I}+1\right)$ th row, i.e. the row below the activated $I$ th block is completed with derivative until its length reaches that of the row above (i.e. $\ell_{I}$ ). The terminology is justified by the fact that the primary Weyl tensor is the gauge-invariant quantity of lowest order in derivatives.
} 

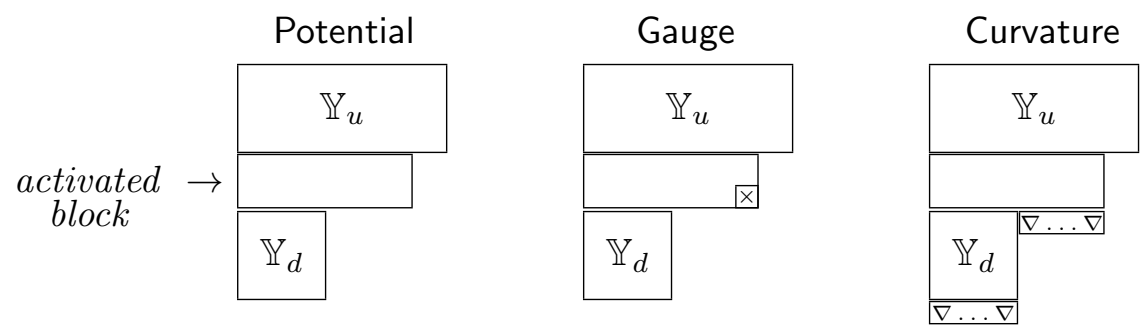

Figure 2. Young diagrams corresponding, from left to right, to a mixed-symmetry massless field, its gauge parameter acting in the isolated middle block with the cross indicating the removed cell, and finally its curvature built by acting with derivatives in the same block. $\mathbb{Y}_{u}$ and $\mathbb{Y}_{d}$ represent arbitrary Young diagrams that can be glued respectively above (up) and below (down) the middle block.

where $\mathbb{Y}_{I}^{\prime(t)}$ is the Young diagram obtained from $\mathbb{Y}$ after having removed $t$ boxes in the last row of the $I$ th block, and where, as in the previous case, all derivatives should be projected so as to reconstruct an object with the symmetry of $\mathbb{Y}$. Notice that the depth of the partially massless field, that is, the number of derivatives involved in its gauge transformation, is now bounded by $s_{I}-s_{I+1}$, i.e. the difference between the length of the $I$ th block and that of the block below (if any). The quantity that we called here curvature is built by acting upon the gauge field with $s_{I}-t+1$ derivatives and projecting it on the symmetry of the Young diagram obtained from $\mathbb{Y}$ by adding a row with $s_{I}-t+1$ boxes under the activated, Ith block. Notice that the depth of a partially massless field can be read off either from its conformal weight, which is $\Delta_{c}=s_{I}+d-p_{I}-t$, or from the difference between the length of the $I$ th block and the next one, in its curvature Young diagram $\mathbb{Y}_{p_{I}+1}$.

Now with this picture in mind, the Young diagrams appearing in (2.13) and (2.14) can be interpreted as follows:

- $\mathbb{Y}_{p}$ corresponds to the curvature of the gauge field, the latter having the $\mathfrak{s o}(d)$ symmetry $\mathbb{Y}=\check{Y}_{p}^{(p)}$, i.e. described by the Young diagram obtained after removing the last row of $\mathbb{Y}_{p}$.

- The last block is activated for this gauge field, i.e. it is subject to gauge transformations generated by a gauge parameter with the symmetry of the Young diagram obtained after removing $t$ boxes, for a depth $t$ partially massless field (keeping in mind that $t=1$ corresponds to the massless case), from the last row of $\check{\mathbb{Y}}_{p}^{(p)}$. It happens to be exactly the shape of the next diagram appearing in (2.13) and (2.14), namely $\check{\mathbb{Y}}_{p}^{(p-1)}$, which therefore corresponds to the gauge parameter of our gauge field.

- Using the same rationale, one can convince oneself that the remaining Young diagram of the type $\check{\mathbb{Y}}_{p}^{(\ell)}, \ell=1, \ldots, p-2$ describe the higher order reducibilities of the gauge parameter with shape $\check{\mathbb{Y}}_{p}^{(p-1)}$.

- The last class of diagrams appearing in (2.13)-(2.14) are of the form $\left(\mathbb{Y}_{p}, \mathbf{1}^{m}\right), m=$ $1, \ldots, r-p$, and describe a chain of Bianchi identities: they are obtained from $\mathbb{Y}_{p}$, the curvature of the gauge field, by adding a box under the last row repeatedly. The vanishing of such tensors would be obtained by acting repeatedly with $\nabla$ on the curvature. 
Notice that, according to this dictionary, only fields where the last block is "activated" are described by UIRs from the exceptional series. This fact complies with the expectation that, in opposition with the anti-de Sitter case where only mixed-symmetry fields whose first block is activated are unitary, in de Sitter unitary mixed-symmetry fields are those whose last block is touched by gauge transformations. This observation is also supported by the fact that, if one forget about unitarity, then irreps in the exceptional series seem to describe mixed-symmetry fields whose activated block is not necessarily the last one (see appendix G). Finally, it appears from the previous discussion that totally symmetric, partially massless, fields are unitary in de Sitter spacetime, in any dimension, as was expected [50]. Notice that the depth- $t$ partially massless fields are those whose curvature Young diagram $\mathbb{Y}_{p}$ last row is shorter than the preceding one, i.e. $s_{p}<s_{p-1}$.

\subsubsection{Discrete series}

Finally, in even spacetime dimensions (i.e. when $d=2 r+1$ ), UIRs in the discrete series arise from reducible generalised Verma module induced by an irrep of $\mathfrak{p}$ of highest-weight $\left[k+\frac{d}{2} ; \vec{s}\right]$ where $k$ is an half-integer that we will rewrite as $k=k^{\prime}-\frac{1}{2}$, with $k^{\prime}$ and positive integer for bosonic fields, ${ }^{10}$ setting a lower bound on the last component of $\vec{s}: 0<k^{\prime} \leqslant s_{r}$, i.e. $\vec{s}$ describes a maximal height Young diagram, as they also can be found in the BGG resolutions detailed in [39] (see appendix D). Their character [36] reads:

$$
\chi_{\left[k+\frac{d}{2} ; \vec{s}\right]}^{\mathrm{dS}}(q, \vec{x})=q^{k^{\prime}+r} \chi_{\vec{s}}^{\mathfrak{s o}(d)}(\vec{x}) \mathcal{P}^{(d)}(q, \vec{x})+\sum_{i=1}^{r}(-1)^{r+1+i} q^{s_{i}+d-i} \chi_{\vec{Y}_{\vec{s}, k^{\prime}}^{(i)}}^{\mathfrak{s o}(d)}(\vec{x}) \mathcal{P}^{(d)}(q, \vec{x})
$$

where $\check{Y}_{\vec{s}, k^{\prime}}^{(i)}$ is the Young diagram obtained from $\vec{s}$ after (i) having removed the $i$ th row as well as one box in all rows below the $i$ th one and (ii) filling the last row with $k^{\prime}-1$ boxes, i.e. ${ }^{11} \check{Y}_{\vec{s}, k^{\prime}}^{(i)}=\left(s_{1}, \ldots, s_{i-1}, s_{i+1}-1, \ldots, s_{r}-1, k^{\prime}-1\right)$. Writing $k^{\prime}=s_{r}-t+1$ with $1 \leqslant t \leqslant s_{r}$, we can recognise the conformal weight of a partially massless mixed-symmetry field with a maximal-height Young diagram (i.e. $p=r$ ) and whose last block is activated, in the exponent of the variable $q$ in the first term: $\Delta_{k}=k^{\prime}+r=s_{r}+d-r-t$. Then, looking at the sum backward (i.e. at the last term with $i=r$ ) we recognise as a second term the conformal weight and Young diagram associated with the gauge parameter of the maximal-height partially massless field: $\Delta_{k}=s_{r}+d-r$ and $\check{Y}_{\vec{s}, k^{\prime}}^{(r)}=\left(s_{1}, \ldots, s_{r-1}, s_{r}-t\right)$. As usual, the removal of $t$ boxes in the last row together with the increase in the conformal weight by $t$ units represents the gauge symmetry enjoyed by the depth- $t$ partially massless field. With this picture in mind, the $r-1$ remaining terms in the above expression are naturally interpreted as the reducibilities of the gauge parameter.

It may seem surprising that, contrarily to the exceptional series, irreps of the discrete series correspond to a description of a massless field only in terms of the potential and its gauge symmetry, and does not involve its curvature. This can be understood a posteriori

\footnotetext{
${ }^{10}$ For fermionic fields, $k^{\prime}$ should be a half-integer, as it is eventually related to the conformal weight of a partially massless field which depends on its spin.

${ }^{11}$ Notice that $t$ has to be an integer (resp. an half-integer) if the components $s_{i}$ 's are also integers (resp. half-integers).
} 
by the fact that those irreps are labelled by a maximal height $\mathfrak{s o}(d)$ Young diagram, and therefore the curvature is described by a Young diagram with $r+1$ rows, which vanishes identically as an $\mathfrak{s o}(d)$ representation and is thus absent in (2.18).

Remark. The 4-dimensional case appears to be somewhat degenerate, in the sense that it can only accommodate totally symmetric fields (the isometry algebra is $\mathfrak{s o}(1,4)$ and therefore the relevant rotation subalgebra is $\mathfrak{s o}(3)$ which has rank 1), hence all massless fields fall in the discrete series (as they are described by maximal height Young diagram). As a consequence, their character only contain the potential part (and not the curvature part) of the gauge field, and therefore are similar to those of massless fields in $\mathrm{AdS}_{4}$.

\subsection{Masslessness: AdS vs dS}

In curved spacetime for fields with spin one or more, the definition of mass (and, therefore, of masslessness) is ambiguous. A standard modern criterion for "masslessness" 12 of fields on de Sitter or anti-de Sitter spacetimes is that the corresponding irrep is not a generalised Verma module (or elementary representation) but arises as a quotient of such modules.

In $(d+1)$-dimensional anti-de Sitter spacetime, (unitary) massive and massless fields are organised quite simply with respect to their conformal weight: given an $\mathfrak{s o}(d)$ highest-weight $\mathbb{Y}$ of height $p-1$ corresponding to the potential, whose first row is of length $s:=\ell_{1}$ and first block of height $h_{1}$, massive fields are those irreps with $\Delta>s+d-h_{1}-1$ and massless fields lie at the boundary of this spectrum, being characterised by $\Delta_{s, h_{1}}:=s+d-h_{1}-1$. The reason for this repartition is the following: for a large conformal weight, $\Delta>\Delta_{s, h_{1}}$, no negative norm vector are present in the module. Then, lowering $\Delta$, some null vectors will appear when reaching the critical value $\Delta_{s, h_{1}}$, that one should get rid of by modding out the submodule they define. Finally, negative state norm start appearing for $\Delta<\Delta_{s, h_{1}}$ so these irreps are non-unitary.

In $(d+1)$-dimensional de Sitter spacetime, there seems to be a similar distribution of (unitary) massive and massless fields as a function of their conformal weight in de Sitter spacetime, with the important difference that both types of field are further split into two subcategories at the group theoretical level. Given the same $\mathfrak{s o}(d)$ highest-weight $\mathbb{Y}$ as considered previously, there is a first continuum of massive fields - the principal series - labelled by a purely complex conformal weight $\Delta_{c}=\frac{d}{2}+i \rho(\rho \in \mathbb{R}$, together with a second marginal continuum of massive fields - the complementary series - with real conformal weight $p<\Delta_{c}<d-p$ (taking into account the partial equivalence between representations with $\Delta_{c}$ and $d-\Delta_{c}$ ). Then at the boundary of the complementary series, $\Delta_{c}=d-p$ and $\Delta_{c}=p$, (partially) massless fields appear as UIRs from the exceptional series. Finally, for $d=2 r+1$, another class of gauge fields is possible, belonging to the discrete series of UIRs. They correspond to (partially) massless fields labelled by Young diagrams of maximal-height.

This repartition is illustrated in the following figure.

Remark. Notice that, because the conformal weight of a field in the exceptional series does not depend on the length of the first row of its Young diagram but on $p$, the height of its first

\footnotetext{
${ }^{12}$ This criterion has the advantage to incorporate in a natural way the partially massless fields.
} 


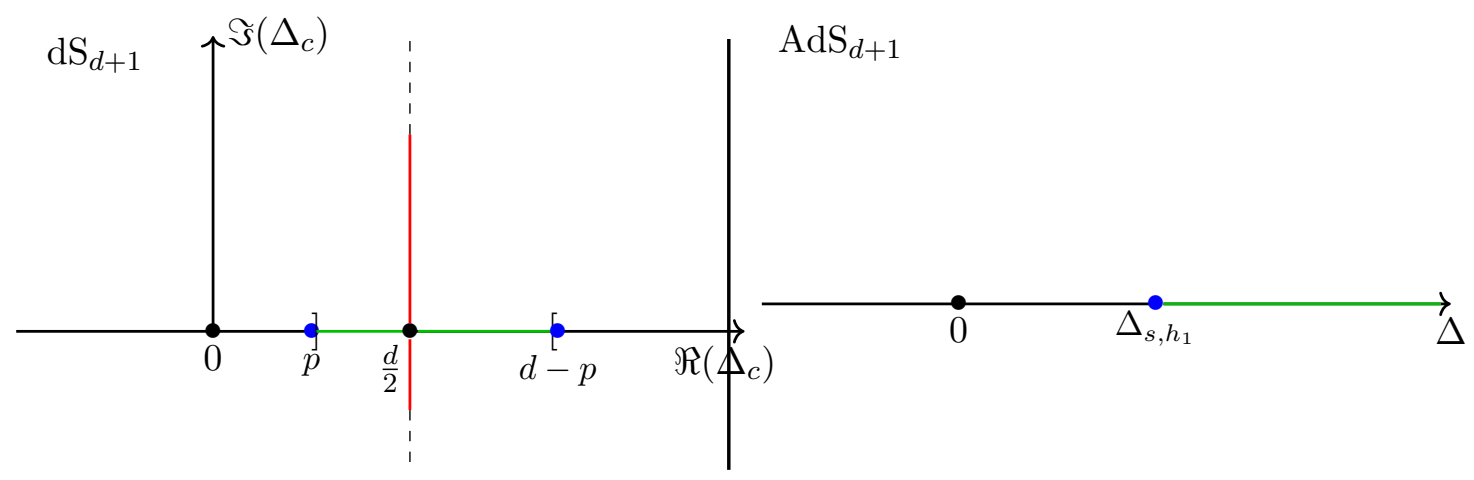

Figure 3. Repartition of massive and massless fields in $\mathrm{dS}_{d+1}$ (left) and $\mathrm{AdS}_{d+1}$ (right) as a function of the conformal weight $\Delta_{c} / \Delta$, for a fixed diagram $\mathbb{Y}$ of total height $p-1$, and first block of height $h_{1}$ and length $s$. On the left / de Sitter side, massive field in the principal and complementary series are depicted respectively by a red and a green line, the massless field is represented by a blue dot. On the right / anti-de Sitter side, massive fields correspond to the green line and the massless fields are the blue dots.

column, the difference between massless and partially massless fields with the same spin is no longer encoded in the conformal weight of the two corresponding representations, like in $\mathrm{AdS}_{d+1}$, but into the Young diagram labeling the irreps. As this diagram corresponds to that of the curvature of the field, a massless and a partially massless field are labelled by a Young diagram whose last row are of different length but of same height, hence both are unitary in $\mathrm{dS}_{d+1}$. In contradistinction, in $\mathrm{AdS}_{d+1}$ the conformal weight of a partially massless field is lower than that of the corresponding massless field and therefore falls below the unitarity bound.

\section{$3 \quad$ Flat limit}

One can recover from the (anti-)de Sitter spacetime $(\mathrm{A}) \mathrm{dS}_{d+1}$ the flat Minkowski spacetime $\mathscr{M}_{d+1}$ by just sending its curvature radius to infinity, $R \rightarrow \infty$, or equivalently by sending to zero the reduced cosmological constant $\lambda^{2}:=-\sigma \frac{2 \Lambda}{d(d-1)}$ where $\sigma=-\Lambda /|\Lambda|$, making this quantity always positive for both sign of the cosmological constant $\Lambda$ (since $\sigma=-1$ corresponds to $\mathrm{dS}_{d+1}$, and $\sigma=+1$ to $\mathrm{AdS}_{d+1}$ ). The flat limit $\lambda=1 / R \rightarrow 0$ corresponds to a contraction of the $(\mathrm{A}) \mathrm{dS}_{d+1}$ isometry algebras to that of Minkowski spacetime, i.e. the Poincaré algebra $\mathfrak{i s o}(1, d)=\mathfrak{s o}(1, d) \notin \mathbb{R}^{d+1}$. Indeed, exhibiting the Lorentz subalgebra, common to these three isometry algebras, they can be presented as:

$$
\left[M_{a b}, M_{c d}\right]=i \eta_{b c} M_{a d}+\ldots, \quad\left[M_{a b}, P_{c}\right]=2 i \eta_{c[b} P_{a]}, \quad\left[P_{a}, P_{b}\right]=i \sigma \lambda^{2} M_{a b}
$$

It is clear from this presentation that sending the inverse radius $\lambda$ of (A)dS to zero, the transvection generators $P_{a}$ become the usual flat spacetime translation generators, which span the abelian ideal $\mathbb{R}^{d+1}$ of the Poincaré algebra. On the (A)dS side, one of these generators belongs to the Cartan subalgebra, and the "energy" label $\left(\Delta_{c}\right.$ or $\Delta$ for respectively $\mathrm{dS}_{d+1}$ or $\left.\mathrm{AdS}_{d+1}\right)$ is the eigenvalue of this particular generator. However, on the flat side, 
there is no longer a Cartan subalgebra, since the Poincaré algebra is not semisimple but a semi-direct sum.

A heuristic way to translate this feature on the characters is to rescale the variable carrying the weight coming from the corresponding transvection generator:

$$
q \rightarrow e^{-\iota \beta \lambda}
$$

with $\iota:=\sqrt{\sigma}$ and $\beta \in \mathbb{R}$ some constant (that we could sometimes interpret as the inverse temperature in the case of negative cosmological constant) with dimension of length; and then to send $\lambda \rightarrow 0$ :

$$
\chi_{\left[\Delta_{(c)} ; \vec{s}\right]}^{(\mathrm{A}) \mathrm{dS}}\left(e^{-\iota \beta \lambda}, \vec{x}\right) \underset{\lambda \rightarrow 0}{\rightsquigarrow} \sum_{[m ; \vec{\sigma}] \in \Sigma\left(\left[\Delta_{(c)} ; \vec{s}\right]\right)} \chi_{[m ; \vec{\sigma}]}^{\text {Poinc. }}(\beta, \vec{x}),
$$

where $[m ; \vec{\sigma}]$ denotes a UIR of the Poincaré group labelled by its mass $m$ and a little group (i.e. $\mathrm{SO}(d)$ for massive irreps, $\mathrm{SO}(d-1)$ for massless helicity ones) highest-weight $\vec{\sigma}$ and $\Sigma\left(\left[\Delta_{(c)}, \vec{s}\right]\right)$ denotes the set of Poincaré irreps resulting from the flat limit (or contraction) of the (A)dS representation labelled by $\left[\Delta_{(c)}, \vec{s}\right]$. Before going into more details on the de Sitter case, which is the main purpose of the present paper, we will start by revisiting the by-now well understood case of mixed-symmetry fields in anti-de Sitter spacetime whose flat limit is controlled by the Brink-Metsaev-Vasiliev (BMV) mechanism.

\subsection{Anti-de Sitter case: the Brink-Metsaev-Vasiliev mechanism}

Brink, Metsaev and Vasiliev conjectured in [23] that a single massless mixed-symmetry fields in anti-de Sitter spacetime is mapped to a set of mixed-symmetry massless fields in flat space; conjecture later proven in [12, 13, 24].

Consider a unitary mixed-symmetry gauge field on $\mathrm{AdS}_{d+1}$ of symmetry characterised by the conformal weight $\Delta_{\ell_{1}, h_{1}}:=\ell_{1}+d-h_{1}-1$ and the Young diagram $\mathbb{Y}=\left(\ell_{1}^{h_{1}}, \ell_{2}^{h_{2}}, \ldots, \ell_{B}^{h_{B}}\right)$ where $\ell_{I}^{h_{I}}$ represents the $I$ th block of length $\ell_{I}$ and height $h_{I}$, and $B$ stands for the number of blocks of the diagram. The total height of $\mathbb{Y}$ is $p=\sum_{I=1}^{B} h_{I}$. The flat limit of this single massless mixed-symmetry field on $\mathrm{AdS}_{d+1}$ is the following:

$$
\mathbb{Y}=\left(\ell_{1}^{h_{1}}, \ell_{2}^{h_{2}}, \ldots, \ell_{B}^{h_{B}}\right) \underset{\lambda \rightarrow 0}{\longrightarrow}\left\{\left(\ell_{1}^{h_{1}}, \ell_{2}^{h_{2}-1}, \ell_{2}-n_{2}, \ldots, \ell_{B}^{h_{B}-1}, \ell_{B}-n_{B}\right)\right\}
$$

where the set of massless fields on $\mathscr{M}_{d+1}$ is determined by the numbers $n_{I}$ of boxes removed from the $\ell_{I}$ th column with $n_{1}=0$ and

$$
0 \leqslant n_{I} \leqslant \ell_{I}-\ell_{I+1}, \quad \forall I \in\{2,3, \cdots, B\} .
$$

This limit is essentially ${ }^{13}$ a branching rule of the orthogonal group: on the $\operatorname{AdS}_{d+1}$ side, the spin is given by the highest-weight of the $\mathfrak{s o}(d)$ subalgebra of $\mathfrak{s o}(2, d)$ whereas in flat spacetime, the spin is given by the highest-weight of the orthogonal (sub)algebra of the little algebra, that is $\mathfrak{s o}(d-1)$ for massless fields in $(d+1)$-dimensional Minkowski space. When

\footnotetext{
${ }^{13}$ The important distinction with a genuine branching rule of the orthogonal group is that the first block is not touched here.
} 
performing the flat limit from $\mathrm{AdS}_{d+1}$ to $\mathscr{M}_{d+1}$, one basically trades the energy/conformal weight for the mass which is obviously zero for massless fields, meaning that they are entirely characterised by their spin. As a consequence, one needs to relate the $\mathfrak{s o}(d)$ part of the representation of $\mathfrak{s o}(2, d)$ leftover after having sent $\lambda$ to zero by branching them onto $\mathfrak{s o}(d-1)$ in order to have a proper interpretation in terms of flat massless fields. To understand the structure of the massless mixed-symmetry representations of $\mathfrak{s o}(2, d)$ in more details, it is quite convenient to have a look at their characters (recalled in appendix B):

$$
\chi_{\left[\Delta_{s, h_{1}} ; \vec{s}\right]}^{\operatorname{AdS}}(q, \vec{x})=q^{\Delta_{s, h_{1}}}\left(\chi_{\vec{s}}^{\mathfrak{s o}(d)}(\vec{x})+\sum_{k=1}^{h_{1}}(-q)^{k} \chi_{\vec{s}_{k}}^{\mathfrak{s o}(d)}(\vec{x})\right) \mathcal{P}^{(d)}(q, \vec{x}) .
$$

The above formula should be read as follows: the module corresponding to a massless mixed-symmetry field in $\operatorname{AdS}_{d+1}$ described by the $\mathfrak{s o}(d)$ highest-weight $\vec{s}$ is obtained by a succession of quotients of generalised Verma modules with $\mathfrak{s o}(d)$ highest-weight $\vec{s}_{k}$ $\left(k=1, \ldots, h_{1}\right)$ obtained from the Young diagram $\vec{s}$ by removing from it the last box on the $k$ last rows in the first block (of height $h_{1}$ ), and increasing the conformal weight by one unit each time a box is removed. This structure is the group-theoretical description underlying the gauge symmetry available for unitary mixed-symmetry fields: they have gauge (for gauge) parameters with the symmetry of each of the $\left(h_{1}-1\right)$ diagrams in the chain obtained from removing a box from the previous diagram. Schematically, this can be depicted as:

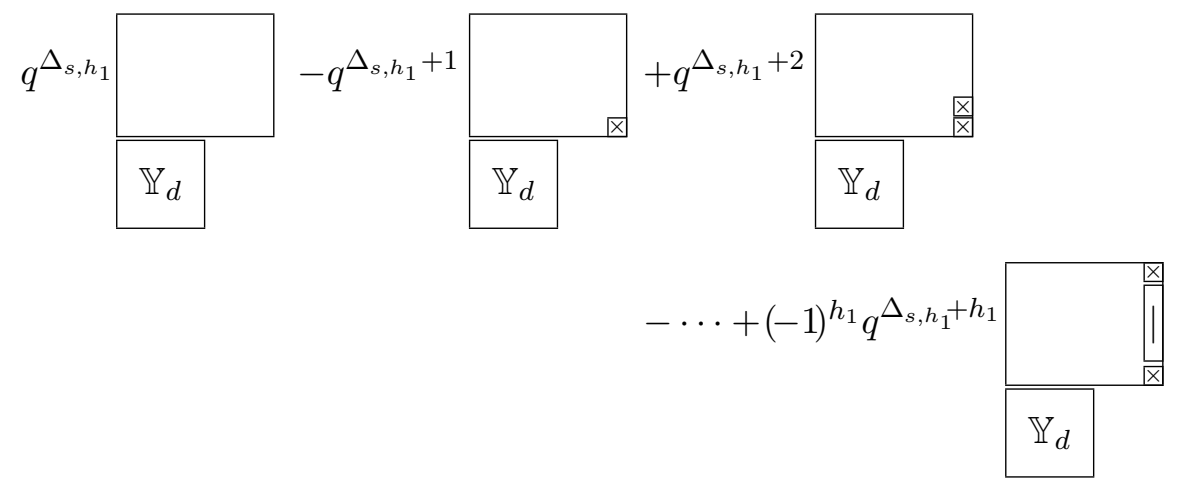

Now, as mentioned above, when taking the flat limit this becomes an alternated sum of $\mathfrak{s o}(d)$ characters. Branching each one of these $h_{1}$ diagrams will produce a number of $\mathfrak{s o}(d-1)$ Young diagrams but this precise sequence is such that only those obtained by deleting boxes in the last rows (until reaching the length of the row just below) in each one of the blocks except the first one. Indeed, branching the first diagram will yield:

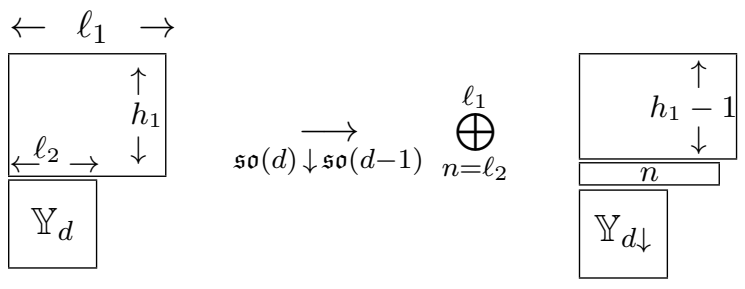


where $\ell_{1}$ is the length of the first block, $\ell_{2}$ is the length of the first row of the second block. The diagram below the first block is represented by $\mathbb{Y}_{d}$, while $\mathbb{Y}_{d \downarrow}$ represents all diagrams obtained from branching $\mathbb{Y}_{d}$. Branching the second Young diagram in $(*)$ will produce exactly the same sum of diagrams, but with $n$ running now from $\ell_{2}$ to $\ell_{1}-1$ instead of $\ell_{1}$. As a consequence, only the diagrams where the first block is left intact and the second is branched onto $\mathfrak{s o}(d-1)$ will survive, which is exactly what the BMV limit tells us. At this stage, one has to notice that the branching of the second diagram will also produce another sum of diagrams, similar to the previous one with $n=\ell_{2}, \ldots, \ell_{1}-1$ but where one extra box is removed in the first block, at the $\left(h_{1}-1\right)$ th row. It turns out that those diagrams will be suppressed when branching the third diagrams, and this mechanism of cancellation will repeat itself until the last (the $h_{1}$ th) diagram, so that in the end one is left only with diagrams produced by the branching rule of $\mathfrak{s o}(d)$ onto $\mathfrak{s o}(d-1)$ except that the first block is intact.

Example 1. Let us consider the example of a mixed-symmetry field in $\mathrm{AdS}_{d+1}$ (in dimension greater or equals to 8$)$ with $\vec{s}=(s, s, 2,1,0, \ldots, 0)$, and look at its flat limit. From the above discussion, it appears that it contracts to the following sequence of $\mathfrak{s o}(d)$ representation:

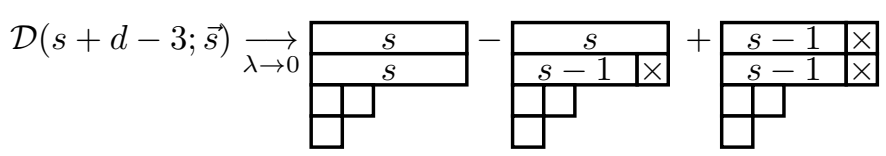

where the boxes containing a $\times$ symbol should be considered as absent (this notation is intended to remind us of the fact that these quotients signify the presence of gauge symmetry). When branching the $\mathfrak{s o}(d)$ Young diagrams onto $\mathfrak{s o}(d-1)$ ones, one produces all Young diagrams obtained by deleting boxes in the last row of each block, until reaching the length of the next rows. For instance, the first diagram branches as:

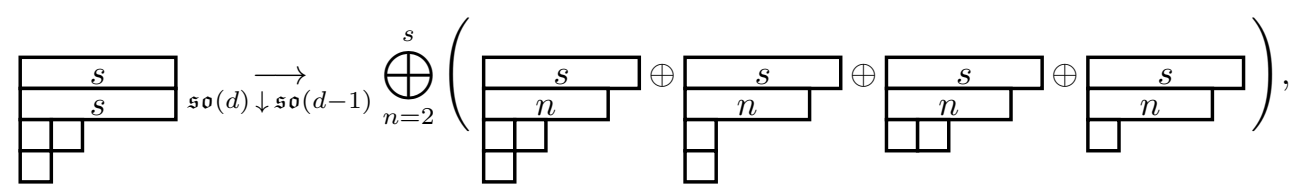

whereas the second and third diagrams yield:

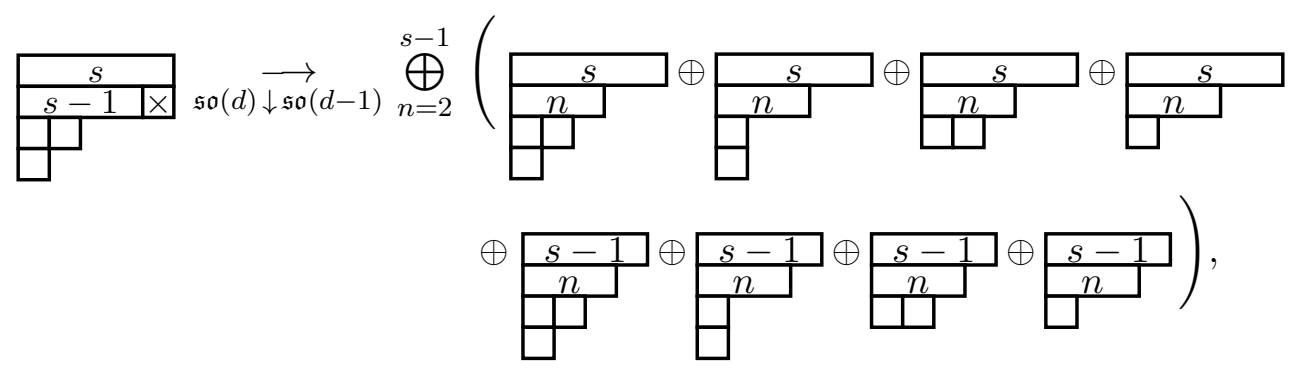

and

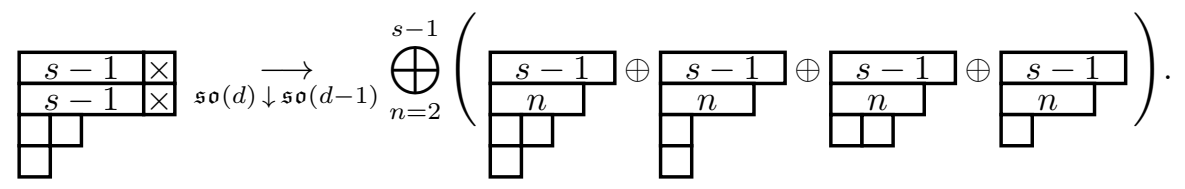


The first line of diagrams in (3.9) obtained after branching the second diagram in the original sequence cancels all those appearing in (3.8), the branching of the first diagram of the sequence, with less than $s$ boxes in the second line, i.e. where the first block is left untouched. The second line of diagrams in (3.9) is identical to those appearing in (3.10), the branching of the third diagram of the sequence, thereby leaving as expected all Young diagrams obtained from branching the original $\mathfrak{s o}(d)$ Young diagram $(s, s, 2,1,0, \ldots, 0)$ onto $\mathfrak{s o}(d-1)$ leaving the first block (composed of the first two rows in this case) intact.

\subsection{Principal and complementary series}

It was shown in [37] that the principal series of representations of the Lorentz group $\mathrm{SO}(1, d+1)$ contracts to the direct sum of two massive representations of the Poincaré group $\operatorname{ISO}(1, d)$ of left and right chirality (when it exists, i.e. for $d=2 r$ ), where the mass is given by $\rho$. In practice, we consider the limit process (3.3), keeping finite the product $\lambda \rho=m$ (in accordance with [37]) :

$$
\begin{aligned}
\chi_{\left[\frac{d}{2}+i \rho ; \vec{s}\right]}^{\mathrm{dS}}(q, \vec{x}) & =q^{d / 2} \mathcal{P}^{(d)}(q, \vec{x})\left(q^{i \rho} \chi_{\overrightarrow{s_{+}}}^{\mathfrak{s o}(d)}(\vec{x})+q^{-i \rho} \chi_{\overrightarrow{s_{-}}}^{\mathfrak{s o}(d)}(\vec{x})\right) \\
& \underset{\lambda \rightarrow 0}{\longrightarrow} \chi_{[m ; \vec{s}]}^{\text {Poinc. }}(\beta, \vec{x})=\left(e^{-\beta m} \chi_{\vec{s}_{+}}^{\mathfrak{s o}(d)}(\vec{x})+e^{\beta m} \chi_{\vec{s}_{-}}^{\mathfrak{s o}(d)}(\vec{x})\right) \mathscr{P}^{(d)}(\vec{x})
\end{aligned}
$$

and

$$
\mathscr{P}^{(d)}(\vec{x}):=\prod_{i=1}^{r} \frac{1}{\left(1-x_{i}\right)\left(1-x_{i}^{-1}\right)}\left\{\begin{array}{cl}
1 & \text { if } d=2 r, \\
\left.\frac{1}{1-\alpha}\right|_{\alpha \rightarrow 1} & \text { if } d=2 r+1,
\end{array}\right.
$$

The resulting expression coincides with the Poincaré characters computed in any dimensions in [27], reviewed in appendix E. The situation is similar for the complementary series of representations, where $\Delta_{c}=\frac{d}{2}+c\left(0<|c|<\frac{d}{2}-p\right)$ except that the product $\lambda c \underset{\lambda \rightarrow 0}{\longrightarrow} 0$ vanishes in the flat limit, so one should set $m=0$ in (3.12) and branch the $\mathfrak{s o}(d)$ characters appearing onto $\mathfrak{s o}(d-1)$ (using the branching rules for the orthogonal algebra recalled in appendix $\mathrm{F}$ ).

\subsection{Exceptional series}

The flat limit of UIRs in the exceptional series is a bit more subtle, but at the same time richer. It is to be excepted, if our identification of this series of irreps with massless fields in de Sitter spacetime is correct: having the BMV mechanism in mind, one would anticipate that the spectrum of massless fields in flat space resulting from the flat limit of a mixedsymmetry field in de Sitter spacetime to be composed of a plethora of fields falling into irreps of $\mathfrak{s o}(d-1)$ related to those appearing in the branching rule of the $\mathfrak{s o}(d)$ Young diagram of the original field. In order to see if these expectations are met, we will perform the flat limit of the characters slightly differently than before: after having set $q=1$, or equivalently sent $\lambda \rightarrow 0$, we will branch all $\mathfrak{s o}(d)$ irreps onto $\mathfrak{s o}(d-1)$, as it characterises entirely the massless Poincaré irreps of helicity type. 


\subsubsection{Even spacetime dimensions}

For $d=2 r+1$, the flat limit of (2.13) yields:

$$
\chi_{\left[d-p ; \mathbb{Y}_{p}\right]}^{\mathrm{dS}}(q, \vec{x}) \underset{\lambda \rightarrow 0}{\longrightarrow} \sum_{\sigma_{1}=s_{2}}^{s_{1}} \sum_{\sigma_{2}=s_{3}}^{s_{2}} \ldots \sum_{\sigma_{p-1}=s_{p}}^{s_{p-1}} \chi_{\left(\sigma_{1}, \ldots, \sigma_{p-1}\right)}^{\mathfrak{s o}(2 r)}(\vec{x}) \mathscr{P}^{(d)}(\vec{x})
$$

Proof. After having set $q=1$ in (2.13), only the following alternating sum of $\mathfrak{s o}(d)$ characters is left:

$$
\chi_{\left[d-p ; \mathbb{Y}_{p}\right]}^{\mathrm{dS}}(q, \vec{x}) \underset{\lambda \rightarrow 0}{\longrightarrow} \sum_{\ell=0}^{p-1}(-1)^{\ell} \chi_{\overleftarrow{\mathbb{Y}}_{p}^{(p-\ell)}}^{\mathfrak{s o}(2 r+1)}(\vec{x}) \mathscr{P}^{(d)}(\vec{x})
$$

where $\check{\mathbb{Y}}_{p}^{(i)}$ was defined in (2.12). Notice that we deliberately used $\check{\mathbb{Y}}_{p}^{(p-\ell)}$ instead of $\check{\mathbb{Y}}_{p}^{(\ell)}$ in the above sum so that the first diagram is the one where the last row was deleted, and consequently the last diagram is the one where the first row was removed. As mentioned previously, in order to figure out the actual field content in flat spacetime, one should branch these diagrams onto $\mathfrak{s o}(2 r)$, the massless little algebra. Because the branching rules for $\mathfrak{s o}(2 r+1)$, given in appendix $\mathrm{F}$, do not involve any additional factors on top of the characters of the irreps appearing in the branching (contrarily to the $\mathfrak{s o}(2 r)$ case), we can trade the characters for the corresponding Young diagrams without loss of information. We will look at the Young diagrams appearing in (3.15) in three groups: we will start by treating the first two diagrams together, then we will look at the last two diagrams, and finally an arbitrary triplet of consecutive diagrams appearing in the above alternate sum.

Let the last three rows (the $(p-2)$ th, $(p-1)$ th and $p$ th) of $\mathbb{Y}_{p}$ be respectively of length $s, t$ and $v$. Starting with the first two diagrams in (3.15), i.e. $\check{\mathbb{Y}}_{p}^{(p)}$ and $\check{\mathbf{Y}}_{p}^{(p-1)}$ and branching them onto $\mathfrak{s o}(d-1)$, we obtain on the one hand for $\check{\mathbb{Y}}_{p}^{(p)}$ :

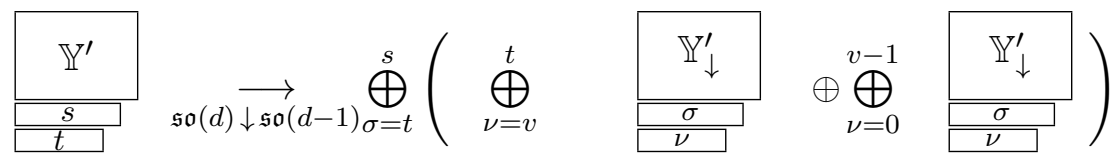

where $\mathbb{Y}^{\prime}$ designates the first $p-3$ rows from the Young diagram of total height $p$ that we are considering and $\mathbb{Y}_{\downarrow}^{\prime}$ all the Young diagrams onto which it branches; and on the other hand $\check{Y}_{p}^{(p-1)}$ :

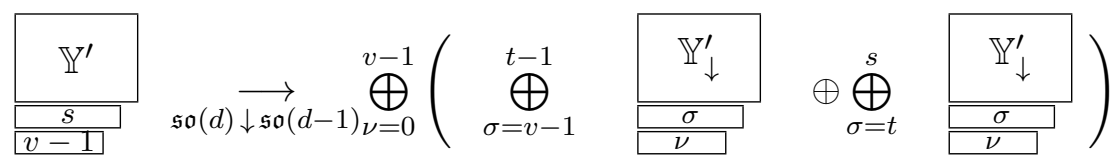

The second parts of the above branched diagrams in figure A and B are common to both of them, and therefore will disappear in the alternating sum (3.15). The first part of the branching from the first diagram describes exactly the field content left after the flat limit, and indeed, we will see that the other parts all cancel each other.

Next, we can have a look at the last two diagrams in the sum (3.15), which both have the form: 


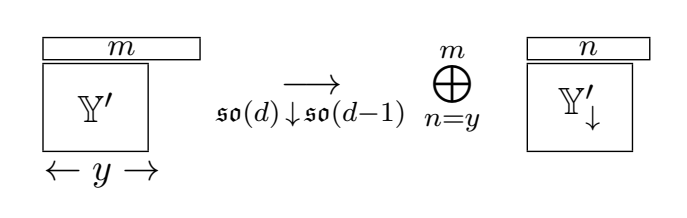

with respectively $m=s_{2}-1$ (for the last diagram $\check{Y}_{p}^{(1)}$ ) and $m=s_{1}$ (for the second to last diagram $\check{Y}_{p}^{(2)}$ ), and where now $\mathbb{Y}^{\prime}$ is the Young diagram made out of the $p-2$ last rows of $\breve{Y}_{p}^{(1)}$ (or $\check{\mathbb{Y}}_{p}^{(2)}$, as they only differ by their first row), and $y=s_{3}-1$ is the length of the first row of $\mathbb{Y}^{\prime}$ for these two diagrams. Because the first row of the last diagram in (3.15) is shorter than the one of the preceding diagram (since $s_{2}-1<s_{1}$ ), all the diagrams resulting from the branching of the last diagram $\check{Y}_{p}^{(1)}$ will also be a part of the branching of the preceding diagram $\check{\mathbb{Y}}_{p}^{(2)}$. Hence, all diagrams produced by the branching of the last one in (3.15) are cancelled by the branching of the second to last one.

Finally, let us consider a triplet of diagrams appearing in the sum (3.15):
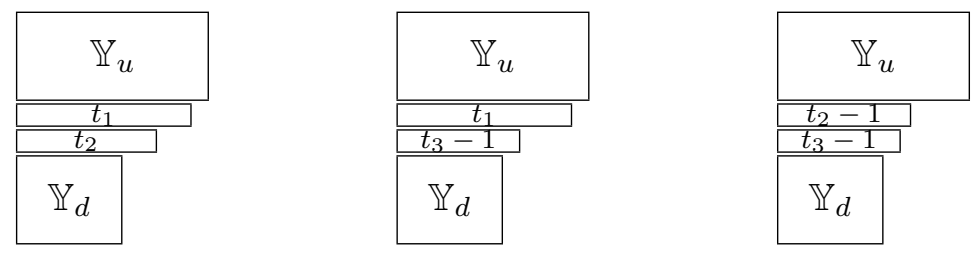

The second diagram in this triplet branches as follows:

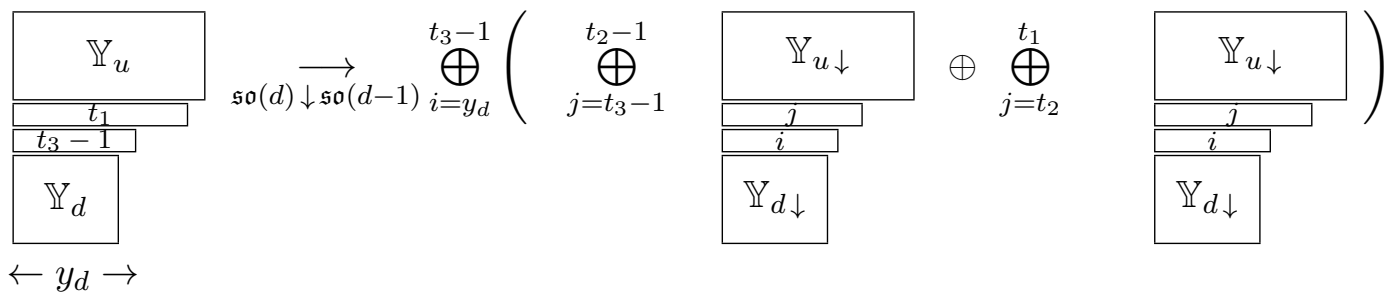

where $y_{d}$ is the length of the first row of $\mathbb{Y}_{d}$. Now one can notice that the second part of this sum will be contained in the branching of the first diagram of the above triplet, whereas the first part of the sum will be contained in the branching of the third diagram in the triplet. As a consequence, every diagrams in the sequence (3.15), obtained by branching onto $\mathfrak{s o}(d-1)$, is cancelled by those coming from the branching of the preceding and following diagram, leaving only those announced above (coming from branching the first diagram in (3.15)).

As explained in appendix E, the characters of massless helicity Poincaré UIRs, in even $d+1=2(r+1)$ dimensions have the form:

$$
\chi_{[0 ; \mathbb{Y}]}^{\text {Poinc. }}(\vec{x})=\chi_{\mathbb{Y}}^{\mathfrak{s o}(2 r)}(\vec{x}) \mathscr{P}^{(d)}(\vec{x})
$$


hence the character obtained from the flat limit of the character of an UIR in the exceptional series of $\mathfrak{s o}(1, d+1)$ associated to an $\mathfrak{s o}(d)$-weight $\mathbb{Y}_{p}=\left(s_{1}, \ldots, s_{p}\right)$ can be rewritten as:

$$
\chi_{\left[d-p ; \mathbb{Y}_{p}\right]}^{\mathrm{dS}}(q, \vec{x}) \underset{\lambda \rightarrow 0}{\longrightarrow} \sum_{\mathbb{Y}^{\prime} \in \Sigma\left(\mathbb{Y}_{p}\right)} \chi_{\left[0 ; \mathbb{Y}^{\prime}\right]}^{\text {Poinc. }}(\vec{x})
$$

with

$$
\Sigma\left(\mathbb{Y}_{p}\right):=\left\{\mathbb{Y}^{\prime}=\left(\sigma_{1}, \ldots, \sigma_{p-1}\right) \mid s_{i+1} \leqslant \sigma_{i} \leqslant s_{i}, i \in\{1,2, \cdots, p-1\}\right\},
$$

describing the spectrum of massless fields appearing in the flat limit. Just as the BMV spectrum in $\mathrm{AdS}_{d+1}$, this set of fields is a truncation of the branching rule of the Young diagram of the gauge potential, i.e. $\check{Y}_{p}^{(p)}$.

- Massless fields: in the particular case of massless fields, one block is left untouched as in the BMV case, but this time it is the last block instead of the first one. Indeed, the Young diagram $\mathbb{Y}_{p}$ describes the shape of the curvature of the gauge field with symmetry $\check{Y}_{p}^{(p)}$, thus the last row has the same length as the previous, i.e. $s_{p}=s_{p-1}$ for massless fields. As a consequence, no box can be removed in the last row of $\check{Y}_{p}^{(p)}$, and its last block is "protected".

In order to emphasise the analogy with the BMV mechanism, the spectrum $\Sigma\left(\mathbb{Y}_{p}\right)$ of massless fields in flat spacetime can be rewritten in a way closer to (3.4) so as to make explicit the blocks of the Young diagram of the massless fields: let $\check{Y}_{p}^{(p)}=$ $\left(\ell_{1}^{h_{1}}, \ldots, \ell_{B}^{h_{B}-1}\right)$, then

$$
\begin{array}{r}
\Sigma\left(\mathbb{Y}_{p}\right)=\left\{\mathbb{Y}^{\prime}=\left(\ell_{1}^{h_{1}-1}, n_{1}, \ldots, \ell_{B-1}^{h_{B-1}-1}, n_{B-1}, \ell_{B}^{h_{B}-1}\right)\right. \\
\left.\ell_{I+1} \leqslant n_{I} \leqslant \ell_{I}, I \in\{1,2, \cdots, B-1\}\right\} .
\end{array}
$$

- Partially massless fields: for depths $t$ strictly higher than one, the situation is similar, up to a minor modification: additional fields can contribute to the above flat spacetime spectrum, namely massless fields with Young diagrams of the same shape as those contained in $\Sigma\left(\mathbb{Y}_{p}\right)$ and in which up to $t-1$ boxes were removed on the last line. More precisely, the spectrum of fields is given by the set:

$$
\begin{aligned}
\Sigma\left(\mathbb{Y}_{p} ; t\right)=\left\{\mathbb{Y}^{\prime}=\left(\ell_{1}^{h_{1}-1}, n_{1}, \ldots, \ell_{B-1}^{h_{B-1}-1}, n_{B-1}, \ell_{B}^{h_{B}}-k\right) \mid\right. \\
\\
\left.\ell_{I+1} \leqslant n_{I} \leqslant \ell_{I}, I \in\{1,2, \cdots, B-1\}, k=0,1, \ldots, t-1\right\} .
\end{aligned}
$$

In particular, this proves what was conjectured in eq. (3.78) of [13].

\subsubsection{Odd spacetime dimensions}

Unfortunately, for even $d$ the situation is not as neat as the previous one. It seems that taking the flat limit at the character level in the same fashion as was done for odd dimensions previously does not produce a natural spectrum of fields in flat space. Indeed, in odd 
spacetime dimension, the flat limit of (2.14) yields:

$$
\begin{aligned}
\chi_{\left[d-p ; \mathbb{Y}_{p}\right]}^{\mathrm{dS}}(q, \vec{x}) \underset{\lambda \rightarrow 0}{\longrightarrow}(-)^{r-p} \sum_{k=1}^{r} \mathcal{A}_{k}^{(r)}(\vec{x})\left(2-\xi_{k}(1)\right) \sum_{\sigma_{1}=s_{2}}^{s_{1}} \\
\ldots \sum_{\sigma_{p-1}=s_{p}}^{s_{p-1}} \sum_{\sigma_{p}=1}^{s_{p}} \chi_{\left(\sigma_{1}, \ldots, \sigma_{p}, \mathbf{1}^{r-1-p}\right)}^{\mathfrak{s o}(2 r-1)}\left(\hat{\vec{x}}_{k}\right) \mathscr{P}^{(2 r)}(\vec{x})
\end{aligned}
$$

where $\xi_{k}$ and $\hat{\vec{x}}_{k}$ are defined in appendix $\mathrm{F}$.

Let us first show how it is obtained, before discussing its significance (or present lack thereof).

Proof. After setting $q=1$ in (2.14), what is left is the following sum of $\mathfrak{s o}(2 r)$ characters:

$$
\begin{aligned}
\chi_{\left[d-p ; \mathbb{Y}_{p}\right]}^{\mathrm{dS}}(q, \vec{x}) \underset{\lambda \rightarrow 0}{\longrightarrow} 2 \sum_{n=0}^{r-p-1}(-)^{n} \chi_{\left(\mathbb{Y}_{p}, \mathbf{1}^{n}\right)}^{\mathfrak{s o}(2)}(\vec{x}) \mathscr{P}^{(2 r)}(\vec{x}) \\
+(-)^{r-p}\left(\chi_{\left(\mathbb{Y}_{p}, \mathbf{1}_{+}^{r-p}\right)}^{\mathfrak{s o}(2 r)}(\vec{x})+\chi_{\left(\mathbb{Y}_{p}, \mathbf{1}_{-}^{r-p}\right)}^{\mathfrak{s o}(2 r)}(\vec{x})\right) \mathscr{P}^{(2 r)}(\vec{x}) \\
\quad-2 \sum_{\ell=0}^{p-1}(-1)^{\ell} \chi_{\check{\mathbb{Y}}_{p}^{(p-\ell)}}^{\mathfrak{s o}(2 r)}(\vec{x}) \mathscr{P}^{(2 r)}(\vec{x})
\end{aligned}
$$

The second line in the above equation will produce the same expression as in the odddimensional case (though with a multiplicity two) once all irreps of $\mathfrak{s o}(2 r)$ are branched onto $\mathfrak{s o}(2 r-1)$. Therefore, what we need to look is the sequence of $\mathfrak{s o}(2 r)$ Young diagrams appearing in the first line.

Let us start with the first two diagrams and their branching, for $\mathbb{Y}_{p}$ :

$$
\begin{array}{|c|c|}
\hline \mathbb{Y}_{u} & \stackrel{s}{\mathfrak{s o}(d)} \underset{\downarrow \mathfrak{s o}(d-1)}{\longrightarrow} \bigoplus_{n=0} \quad \mathbb{Y}_{u \downarrow} \\
& n
\end{array}
$$

where $\mathbb{Y}_{u}$ represents the Young diagram made out of the $p-1$ first rows of $\mathbb{Y}$, and for $\left(\mathbb{Y}_{p}, 1\right)$

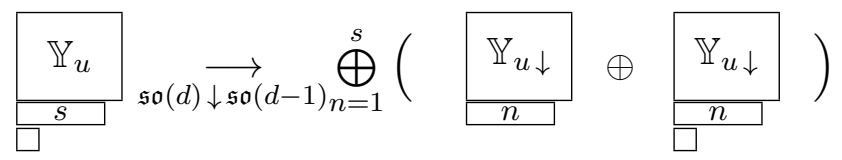

From the branching of the first diagram, only the part with $n=0$ of the sum survives in the reduction of (3.22), that is all diagrams obtained from branching $\mathbb{Y}_{p}$ and removing its last row. This makes all up the diagrams appearing in the flat limit for $d=2 r+1$. They will therefore cancel exactly those coming from branching the second line of (3.22). Just as in the previous odd-dimensional case, the rest of the sequence is such that all other diagrams, when branched, produce a set of diagrams that will, for the most part, be cancelled. Indeed, in general a diagram of the form $\left(\mathbb{Y}_{p}, \mathbf{1}^{m}\right)$ branches as:

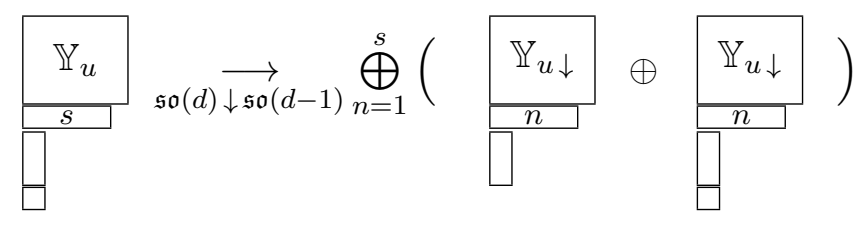


The first sum of diagrams in the right hand side is exactly the second sum of diagrams that appear when branching the Young diagram $\left(\mathbb{Y}_{p}, \mathbf{1}^{m-1}\right)$, therefore it will be cancelled. The last thing to check is that the last diagram, the one with maximal height $r$ will not bring diagrams that cannot be cancelled by previous terms. As the branching process here is to go from $\mathfrak{s o}(2 r)$ to $\mathfrak{s o}(2 r-1)$, a Young diagram with maximal height will branch only onto diagrams where the last row was removed (so that it has the correct height for an $\mathfrak{s o}(2 r-1)$ Young diagram). Concretely:

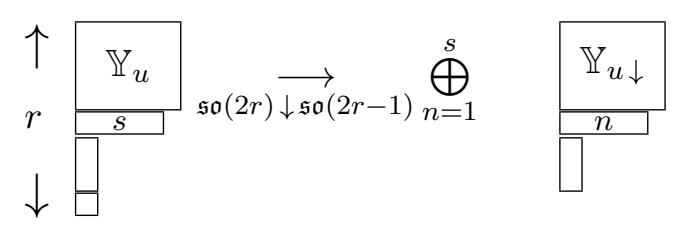

However, taking into account the fact that the branching rule for an $\mathfrak{s o}(2 r)$ character of a Young diagram of maximal height involves an additional factor compared to the nonmaximal Young diagrams, see (F.18), the $\mathfrak{s o}(2 r-1)$ diagrams produced by branching the $\mathfrak{s o}(2 r)$ diagram $\left(\mathbb{Y}_{p}, \mathbf{1}^{r-p}\right)$ are not cancelled, rather they come with a factor $2-\xi(1)$.

In order to illustrate the mechanism explained above, let us detail a concrete case in the example below.

Example 2. Let us consider a massless, totally symmetric, spin- $s$ field in $\mathrm{dS}_{d+1}$ when $d=2 r$, for the sake of simplicity. Its character reads:

$$
\begin{aligned}
\chi_{[d-2 ; s, s]}^{\mathrm{dS}}(q, \vec{x})=\sum_{m=0}^{r-3}( & )^{m}\left(q^{d-2-m}+q^{2+m}\right) \chi_{\left(s, s, \mathbf{1}^{m}\right)}^{\mathfrak{s o}(2 r)} \mathcal{P}^{(d)}(q, \vec{x}) \\
& -2 q^{s+d-2}\left(\chi_{(s)}^{\mathfrak{s o}(2 r)}(\vec{x})-q \chi_{(s-1)}^{\mathfrak{s o}(2 r)}(\vec{x})\right) \mathcal{P}^{(d)}(q, \vec{x}) \\
& +(-)^{r} q^{r}\left(\chi_{\left(s, s, \mathbf{1}_{+}^{r-2}\right)}^{\mathfrak{s o}(2 r)}(\vec{x})+\chi_{\left(s, s, \mathbf{1}_{-}^{r-2}\right)}^{\mathfrak{s o}(2 r)}(\vec{x})\right) \mathcal{P}^{(d)}(q, \vec{x})
\end{aligned}
$$

In the flat limit considered so far in this paper, $q \rightarrow 1$, it becomes:

$$
\begin{aligned}
& \chi_{[d-2 ; s, s]}^{\mathrm{dS}}(q, \vec{x}) \underset{\lambda \rightarrow 0}{\longrightarrow}(\left(2 \sum_{m=0}^{r-3}(-)^{m} \chi_{\left(s, s, \mathbf{1}^{m}\right)}^{\mathfrak{s o}(2 r)}-2\left[\chi_{(s)}^{\mathfrak{s o}(2 r)}(\vec{x})-\chi_{(s-1)}^{\mathfrak{s o}(2 r)}(\vec{x})\right]\right. \\
&\left.+(-)^{r}\left[\chi_{\left(s, s, \mathbf{1}_{+}^{r-2}\right)}^{\mathfrak{s o}(2 r)}(\vec{x})+\chi_{\left(s, s, \mathbf{1}_{-}^{r-2}\right)}^{\mathfrak{s o}(2 r)}(\vec{x})\right]\right) \mathscr{P}^{(d)}(\vec{x})
\end{aligned}
$$

Now using the branching rules derived in appendix F:

$$
\begin{aligned}
\chi_{(s)}^{\mathfrak{s o}(2 r)}(\vec{x})-\chi_{(s-1)}^{\mathfrak{s o}(2 r)}(\vec{x}) & =\sum_{k=1}^{r} \mathcal{A}_{k}^{(r)}(\vec{x})\left(\sum_{\sigma=0}^{s} \chi_{(\sigma)}^{\mathfrak{s o}(2 r-1)}\left(\hat{\vec{x}}_{k}\right)-\sum_{\sigma=0}^{s-1} \chi_{(\sigma)}^{\mathfrak{s o}(2 r-1)}\left(\hat{\vec{x}}_{k}\right)\right) \\
& =\sum_{k=1}^{r} \mathcal{A}_{k}^{(r)}(\vec{x}) \chi_{(s)}^{\mathfrak{s o}(2 r-1)}\left(\hat{\vec{x}}_{k}\right),
\end{aligned}
$$


as was observed in, for instance, [27]. Now turning to the curvature and Bianchi identities contributions:

$$
\begin{aligned}
& \chi_{(s, s)}^{\mathfrak{s o}(2 r)}(\vec{x})=\sum_{k=1}^{r} \mathcal{A}_{k}^{(r)}(\vec{x}) \sum_{\sigma=0}^{s} \chi_{(s, \sigma)}^{\mathfrak{s o}(2 r-1)}\left(\hat{\vec{x}}_{k}\right) \\
& \chi_{\left(s, s, \mathbf{1}^{m}\right)}^{\mathfrak{s o}(2 r)}(\vec{x})=\sum_{k=1}^{r} \mathcal{A}_{k}^{(r)}(\vec{x}) \sum_{\sigma=1}^{s}\left(\chi_{\left(s, \sigma, \mathbf{1}^{m}\right)}^{\mathfrak{s o}(2 r-1)}\left(\hat{\vec{x}}_{k}\right)+\chi_{\left(s, \sigma, \mathbf{1}^{m-1}\right)}^{\mathfrak{s o}(2 r-1)}\left(\hat{\vec{x}}_{k}\right)\right), \quad(m=1, \ldots, r-3) \\
& \chi_{\left(s, s, \mathbf{1}_{+}^{r-2}\right)}^{\mathfrak{s o}(2 r)}(\vec{x})+\chi_{\left(s, s, \mathbf{1}_{-}^{r-2}\right)}^{\mathfrak{s o}(2 r)}(\vec{x})=\sum_{k=1}^{r} \mathcal{A}_{k}^{(r)}(\vec{x}) \xi_{k}(1) \sum_{\sigma=1}^{s} \chi_{\left(s, \sigma, \mathbf{1}^{r-3}\right)}^{\mathfrak{s o}(2 r-1)}\left(\hat{\vec{x}}_{k}\right)
\end{aligned}
$$

Making use of these 3 equations, one ends up with:

$$
\begin{aligned}
& 2 \sum_{m=0}^{r-3}(-)^{m} \chi_{\left(s, s, \mathbf{1}^{m}\right)}^{\mathfrak{s o}(2 r)}+(-)^{r}\left[\chi_{\left(s, s, \mathbf{1}_{+}^{r-2}\right)}^{\mathfrak{s o}(2 r)}(\vec{x})+\chi_{\left(s, s, \mathbf{1}_{-}^{r-2}\right)}^{\mathfrak{s o}(2 r)}(\vec{x})\right] \\
& \quad=\sum_{k=1}^{r} \mathcal{A}_{k}^{(r)}(\vec{x})\left(2 \chi_{(s)}^{\mathfrak{s o}(2 r-1)}\left(\hat{\vec{x}}_{k}\right)+(-)^{r}\left(2-\xi_{k}(1)\right) \sum_{\sigma=1}^{s} \chi_{\left(s, \sigma, \mathbf{1}^{r-3}\right)}^{\mathfrak{s o}(2 r-1)}\left(\hat{\vec{x}}_{k}\right)\right)
\end{aligned}
$$

Putting all the pieces together, the flat limit now reads:

$$
\chi_{[d-2 ; s, s]}^{\mathrm{dS}}(q, \vec{x}) \underset{\lambda \rightarrow 0}{\longrightarrow}(-)^{r} \sum_{k=1}^{r} \mathcal{A}_{k}^{(r)}(\vec{x})\left(2-\xi_{k}(1)\right) \sum_{\sigma=1}^{s} \chi_{\left(s, \sigma, \mathbf{1}^{r-3}\right)}^{\mathfrak{s o}(2 r-1)}\left(\hat{\vec{x}}_{k}\right),
$$

which is, to say the least, confusing, having nothing to do with the quite coherent and expected spectrum produced by the flat limit of $\mathfrak{s o}(1, d+1)$ characters when $d=2 r+1$.

As announced at the beginning of this subsection, this flat limit is a bit puzzling, as it cannot be interpreted naturally as a BMV-type spectrum in de Sitter. Although the characters of exceptional series representations have the same structure (that is, as explained in section 2, it contains information about the gauge fields, its gauge parameter and their reducibility, as well as the curvature and its Bianchi identities), there are two problems arising when considering their flat limit as we proposed:

(i) The two sequences of $\mathfrak{s o}(d)$ Young diagrams appearing in the character and describing on one side the gauge field and its gauge parameter, and on the other side the curvature and its Bianchi identities, both produce the expected spectrum $\Sigma\left(\mathbb{Y}_{p}\right)$ when branched onto $\mathfrak{s o}(d-1)$ but come with a relative minus sign, hence they cancel each other.

(ii) In the "Bianchi" sequence, the presence of maximal height $\mathfrak{s o}(2 r)$ Young diagram of the form $\left(\mathbb{Y}_{p}, \mathbf{1}_{ \pm}^{r-p}\right)$ whose characters, when branched onto $\mathfrak{s o}(2 r-1)$ involve an additional factor $\xi(1)$ with respect to non-maximal Young diagrams (see appendix F). As a consequence, we are left with some maximal height diagrams of $\mathfrak{s o}(2 r-1)$ which have, to our knowledge, no interpretation as massless fields resulting from a flat limit of the original gauge field. 
A possible resolution of these difficulties could be brought by the following argument: as mentioned in section 2, the classification of UIRs - classification that we related to a field theoretic classification of massive and massless fields in de Sitter — was obtained at the Lie group $\mathrm{SO}(1, d+1)$ level. To obtain such a dictionary, we looked for the corresponding UIRs at the Lie algebra $\mathfrak{s o}(1, d+1)$ level, UIRs for which we could write down the corresponding characters. In turn, these characters gave us some insight into the structure of each of these representations. This being said, it is a well known fact that not all Lie algebra representations extend to group representations. Therefore one could speculate that the reason why we did not find the irrep in the known classification of $\mathrm{SO}(1, d+1)$ UIRs that would correspond to the potential module only (i.e. the module made out of the potential, quotiented by its gauge parameter and its higher-order reducibilities) is precisely because this representation of the Lie algebra does not extend to a unitary representation of the Lie group.

If this happens to be correct, then in both odd $(d=2 r)$ and even $(d=2 r+1)$ spacetime dimension, for a (partially) massless field of given $\mathfrak{s o}(d)$ type, we prescribe to consider only the gauge potential part of the module for which the character in dS is exactly the same as the character for $\mathfrak{s o}(2, d)$ irreps corresponding to the same $\mathfrak{s o}(d)$ type. Therefore, the flat limit of the purely potential $\mathfrak{s o}(1, d+1)$ module will produce the sum of $\mathfrak{i s o}(1, d)$ characters corresponding to the BMV spectrum $\Sigma$ we found in (3.19) for the unitary case in dS. For the non-unitary cases in both dS and AdS, see appendix G. The only difference is the protected block for unitary fields (the first one in $\mathrm{AdS}_{d+1}$, the last one in $\mathrm{dS}_{d+1}$ ).

Our above interpretation is supported by the fact that the technique of the proof presented in $[12,13]$ holds for both AdS and dS, irrespectively of the parity of the dimension. Actually, in $[12,13]$ the whole procedure was presented for both signs of the cosmological constant. Only the computations of the critical masses were performed for the AdS signature, though there is nothing that would prevent one to compute the critical masses for the other signature.

\subsection{Discrete series}

The flat limit of UIRs in the discrete series is quite similar to that of exceptional series representations in odd spacetime dimensions. Indeed, the "Bianchi-identity part" (i.e. containing the Young diagrams $\left.\left(\mathbb{Y}_{p}, \mathbf{1}^{m}\right), m=0, \ldots, r-p\right)$ of the character of exceptional series irreps vanishes (due to the factor $q^{\Delta_{c}}-q^{d-\Delta_{c}} \stackrel{q \rightarrow 1}{\rightarrow} 0$ coming in front of it), therefore we are only left with the usual ${ }^{14}$ set of Young diagrams corresponding to the field, its gauge parameter and its reducibility. As a consequence, one has to branch the same type of sequence of $\mathfrak{s o}(d)$ characters as for exceptional series characters in odd spacetime dimensions, with the only difference that here the first Young diagram (corresponding to the massless field itself) is of maximal height. This last specifity does not change the argument presented in the previous section for the flat limit of UIRs in the exceptional series. Thence, we obtain the following flat limit of UIRs in the discrete series or, equivalently, (partially)

\footnotetext{
${ }^{14}$ Usual in the sense that it is the only one $\mathrm{AdS}_{d+1}$ characters appearing and is therefore the part that $\mathrm{dS}_{d+1}$ characters have in common with the former.
} 
massless fields with maximal-height Young diagrams:

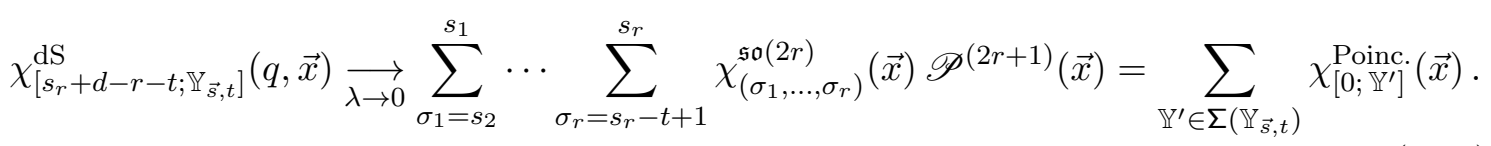

with, upon rewriting $\mathbb{Y}_{\vec{s}, t}$ as $\left(\ell_{1}^{h_{1}}, \ldots, \ell_{B}^{h_{B}}\right)$ to exhibit its various blocks (with $\sum_{I=1}^{B} h_{I}=r$ and $\ell_{B}=s_{r}$ ), the flat space spectrum:

$$
\begin{array}{r}
\Sigma\left(\mathbb{Y}_{\vec{s}, t}\right):=\left\{\mathbb{Y}^{\prime}=\left(\ell_{1}^{h_{1}-1}, \ell_{1}-n_{1}, \ldots, \ell_{B-1}^{h_{B-1}-1}, \ell_{B-1}-n_{B-1}, \ell_{B}^{h_{B}-1}, \ell_{B}-m\right),\right. \\
\left.0 \leqslant n_{I} \leqslant \ell_{I}-\ell_{I+1}, I=1, \ldots, B-1, m=0, \ldots, t-1\right\} .
\end{array}
$$

As previously, it appears that for massless fields (i.e. $t=1$ ) the last block is protected whereas for partially massless fields, the corresponding flat spacetime spectrum can also contain fields where up to $t-1$ boxes are removed from the last block.

\section{Conclusions}

In this paper, we investigated the representation theory of $\mathfrak{s o}(1, d+1)$ and tried to give a field theoretic interpretation of the list of UIRs known for this algebra. We proposed a dictionary between (partially) massless mixed-symmetry fields of arbitrary shape and representations in the exceptional and discrete series, thereby extending and completing the work of $[17,18]$ concerning scalar fields. A byproduct of this identification is to confirm the anticipated unitarity of partially massless fields in de Sitter. More precisely, we found for gauge fields of arbitrary shape, that unitary fields in $\mathrm{dS}_{d+1}$ are those whose gauge symmetry involves the lowest block of their Young diagram. This generalises the analysis of unitarity of mixed-symmetry partially massless fields on de Sitter spacetime from the case of two-column Young diagrams $[51,52]$ to the generic case; see also [53-56] where some types of massive mixed-symmetry fields in (A)dS and various massless limits were analysed starting from Lagrangian formulations.

In the process of studying $\mathfrak{s o}(1, d+1)$ irreps, we were able to derive their character, which gives us some insight into the structure of the corresponding modules. Inspired by the BMV mechanism in anti-de Sitter spacetime [12, 13, 23, 24], we proposed a way of taking the flat limit of those characters and read off the resulting flat spacetime spectrum by recognising characters of the Poincaré group. Although this procedure fails for UIRs in the exceptional series when $d$ is even, this method yields a fairly coherent picture of the flat limit of massless fields in de Sitter. In $\mathrm{AdS}_{d+1}$, the BMV spectrum of unitary massless mixed-symmetry fields in flat spacetime is given by the $\mathfrak{s o}(d)$ branching rules of the field's Young diagram, where the first block, activated by the gauge transformations, is left untouched. A similar situation occurs in de Sitter spacetime, but instead of the upper block being protected, it is now the lowest one that is left untouched when branching the field's Young diagram onto $\mathfrak{s o}(d-1)$. As argued in appendix G, this BMV-type spectrum should hold for generic massless fields, even non unitary ones: mixed-symmetry fields whose block affected by gauge transformations is not the first one in $\mathrm{AdS}_{d+1}$ or the last 
one in $\mathrm{dS}_{d+1}$, should produce in the flat limit all massless field labeled by a Young diagram contained in the branching rule of the original (A) $\mathrm{dS}_{d+1}$ field's diagram, leaving the block activated by the gauge symmetry untouched.

Our proposition should, however, be considered with caution. Despite the quite coherent landscape of fields described by UIRs of $\mathfrak{s o}(1, d+1)$ according to our identifications and the consistent BMV-type spectrum obtained in even spacetime dimensions, the failure to obtain a similar one in odd spacetime dimensions is puzzling, and definitely calls for further investigation. As we explained in section 3, we think that the resolution of this puzzle (namely the fact that from the field theory point of view the parity of the spacetime dimension does not bring any difference in the treatment or behaviour of massless fields, whereas we observe a drastic distinction at the group theoretical level) is the distinction between group and algebra irreps. In order to make contact with the known classification of UIRs of $\mathrm{SO}(1, d+1)$, we had to look at the group irreps which seem to be formulated in terms of the curvature of the massless fields, but at the level of the algebra it may be possible to consider irreps describing only the gauge field (as we are used to in $\mathrm{AdS}_{d+1}$ ). Nevertheless, having at hand this proposed dictionary of $\mathfrak{s o}(1, d+1)$ irreps and the corresponding characters opens several possibilities, such as the construction of a Flato-Fronsdal theorem [57] for de Sitter. The decomposition of the product of two "shortest representations" (that are the Dirac scalar and spinor singletons) into irreducible representations, as a tower of massless spin- $s$ fields in $\mathrm{AdS}_{d+1}$, is at the heart of the higher-spin AdS/CFT correspondence [58, 59]. A similar theorem in de Sitter spacetime would provide a similar kinematical evidence in favour of the proposal [60] of a higher-spin dS/CFT correspondence. Even though we did not find an obvious unitary singleton-type representation in the list of known $\mathfrak{s o}(1, d+1)$ irreps, one would expect that such UIRs exist because of their rôle in the definition of the higher-spin algebra in (A) $\mathrm{dS}_{d+1}$ (see for instance [61-63] for nice overviews), which is insensitive to the signature. In fact, we identified a natural candidate for the contragredient of the singleton representation and their higher-order generalisations [64]. Another potential evidence in this direction is the fact that in $\mathrm{dS}_{4}$, massless totally symmetric fields have the same character as their $\mathrm{AdS}_{4}$ counter part, and therefore summing the characters of massless spin- $s$ fields on all spins will yield the square of the scalar singleton character. It is therefore natural to expect this type of decomposition to remain true in any dimensions.

\section{Acknowledgments}

It is a pleasure to thank Andrea Campoleoni for discussions on the characters of the Poincaré group. We also thank Philippe Spindel for discussions. T.B. is supported by a joint grant "50/50" Université François Rabelais Tours — Région Centre / UMONS. The research of X.B. was supported by the Russian Science Foundation grant 14-42-00047 in association with the Lebedev Physical Institute. 


\section{A Massive scalar field on (anti) de Sitter spacetime}

Let us consider a massive scalar field in de Sitter spacetime, subject to Klein-Gordon's equation:

$$
\left(\nabla_{\mathrm{dS}_{d+1}}^{2}-m^{2}\right) \phi=0
$$

where $\nabla^{2}:=g^{\mu \nu} \nabla_{\mu} \nabla_{\nu}$ is the Laplace-Beltrami operator in $(d+1)$-dimensional de Sitter spacetime. Using the "inflationary coordinates" (covering only half of $\mathrm{dS}_{d+1}$ ), in which the metric looks like:

$$
d s_{\mathrm{dS}_{d+1}}^{2}=-d t^{2}+e^{2 t / R} d \vec{y}^{2}
$$

where $R$ is the curvature radius and $d \vec{y}^{2}$ is the line element of the $d$-dimensional Euclidean space $\mathbb{R}^{d}$, one can obtain a patch similar to the Poincaré patch in $\operatorname{AdS}_{d+1}$ by the following change of coordinate:

$$
\sqrt{\frac{R}{\rho}}=e^{t / R} \Rightarrow d t=-R \frac{d \rho}{2 \rho}
$$

after which the metric has the form:

$$
d s_{\mathrm{dS}_{d+1}}^{2}=R^{2}\left(-\frac{d \rho^{2}}{4 \rho^{2}}+\frac{1}{R \rho} d \vec{y}^{2}\right) .
$$

The only difference with the $\mathrm{AdS}_{d+1}$ case is, as could be expected, the signature of the line element $d \vec{y}^{2}$ and of the coordinate $\rho$. In these coordinates, the Klein-Gordon equation reads

$$
\nabla_{\mathrm{dS}_{d+1}}^{2} \phi(\rho, \vec{y})=\frac{1}{R^{2}}\left[-4 \rho^{2} \partial_{\rho}^{2}+4\left(\frac{d}{2}-1\right) \rho \partial_{\rho}+R \rho \Delta_{\mathbb{R}^{d}}\right] \phi(\rho, \vec{y})=m^{2} \phi(\rho, \vec{y})
$$

with $\Delta_{\mathbb{R}^{d}}=\delta^{i j} \frac{\partial}{\partial y^{i}} \frac{\partial}{\partial y^{j}}$ is the Laplacian on $d$-dimensional Euclidean space. Using the same ansatz as for $\operatorname{AdS}_{d+1}$, i.e.

$$
\phi(\rho, \vec{y})=\rho^{\Delta_{c} / 2} \varphi(\rho, \vec{y})
$$

with $\varphi(\rho, \vec{y})$ a scalar field, well-behaved at the conformal boundary of $\mathrm{dS}_{d+1}$ (located at $\rho \rightarrow 0 \Leftrightarrow t \rightarrow \infty)$. Plugging this into (A.5), and evaluating it at the boundary, we deduce:

$$
(m R)^{2}=\Delta_{c}\left(d-\Delta_{c}\right) \quad \Rightarrow \quad \Delta_{c, \pm}=\frac{d}{2} \pm \sqrt{\frac{d^{2}}{4}-m^{2} R^{2}}
$$

Contrarily to the $\mathrm{AdS}_{d+1}$ case, ${ }^{15}$ it appears that one can have complex conformal weight $\Delta_{c}$ compatible with unitarity when the scalar field is "very massive", i.e. when $m R>d / 2$. In this case, we recognise a conformal weight corresponding to a representation in the principal series: $\Delta_{c}=\frac{d}{2} \pm i \sqrt{m^{2} R^{2}-\frac{d^{2}}{4}}$. For "not-so-massive" fields, i.e. $0 \leqslant m R<d / 2$, the conformal weight is real and within the boundary of the complementary series: $\frac{d}{2}<\Delta_{c}<d$.

\footnotetext{
${ }^{15}$ Because of the signature difference, in $\operatorname{AdS}_{d+1}$ one gets $m^{2}=\Delta(\Delta-d)$ which leads to $\Delta_{ \pm}=\frac{d}{2} \pm$ $\sqrt{\frac{d^{2}}{4}+m^{2} R^{2}}$, therefore $\Delta$ is never complex in the unitary case. For scalar fields satisfying the BreitenlohnerFreedman unitarity bound $(m R)^{2} \geqslant-\frac{d^{2}}{4}[65,66]$ corresponding to $\Delta \geqslant 0$ and real (as eigenvalue of the $\mathcal{U}(1)$ energy operator).
} 
If we have $0 \leqslant m R<d / 2$, we can keep on our analysis in the same manner as in $\mathrm{AdS}_{d+1}$, when performing the holographic reconstruction of a scalar fields (see e.g. [67, 68]). The next step is then to expand $\varphi$ in powers of $\rho$ :

$$
\varphi(\rho, \vec{y})=\sum_{n=0}^{\infty} \rho^{n} \varphi_{n}(\vec{y})
$$

where $\varphi_{n}(\vec{y})$ are also well-behaved fields. By plugging this expansion in (A.5) and using $m^{2}=\Delta_{c}\left(d-\Delta_{c}\right)$, we obtain the following recurrence relation among the modes of $\varphi$ :

$$
2 n\left(2 n+2 \Delta_{c, \pm}-d\right) \varphi_{n}(\vec{y})=R \Delta_{\mathbb{R}^{d}} \varphi_{n-1}(\vec{y})
$$

As in the $\mathrm{AdS}_{d+1}$ case, this recurrence relation can break down if $\Delta_{c}=\frac{d}{2}-\ell$ for some integer $\ell \geqslant 1$, which is possible for $\Delta_{c,-}$ if $\sqrt{\frac{d^{2}}{4}-m^{2} R^{2}}=\ell \in \mathbb{N}_{0}$. In this case, a possible solution is [68] to impose the polywave equation as a constraint $\left(\Delta_{\mathbb{R}^{d}}\right)^{\ell} \varphi_{0}(\vec{y})=0$ on the lowest order term. The power series expansion in this case reads:

$$
\phi(\rho, \vec{y})=\rho^{\Delta_{c,-} / 2}\left(\varphi_{0}(\vec{y})+\rho \varphi_{1}(\vec{y})+\ldots\right)+\rho^{\Delta_{c,+} / 2}\left(\tilde{\varphi}_{0}(\vec{y})+\rho \tilde{\varphi}_{1}(\vec{y})+\ldots\right)
$$

Notice that, as in $\operatorname{AdS}_{d+1}$, see e.g. [68], it is the branch $\varphi$ that is the leading one when $\rho \rightarrow 0$. Recall that in $\mathrm{AdS}_{d+1}$, the fact that this branch was the subleading one toward the boundary, added to the fact that this part of the series exapansion of the field is always a solution to (A.5) leads to the conclusion that, for the space of solution of (A.5) to be an irreducible $\mathfrak{s o}(2, d)$ module, one has to quotient by the subspace of subleading solutions. Effectively, only subleading solutions remain in the module, which thereby defines a (higher-order) singleton: a scalar field propagating no local degrees of freedom in the bulk (i.e. "confined" at the conformal boundary) and defining a boundary conformal scalar obeying the (poly)wave equation $\square^{\ell} \phi=0$. It complies with the fact that one would expect a would-be de Sitter singleton to be the fundamental field of the conformal field theory dual to the higher-spin theory in $\mathrm{dS}_{d+1}$. According to the proposed duality in [60], the field should fall in a non-unitary irrep of $\mathfrak{s o}(1, d+1)$. In our case, the field reconstructed previously should belong to the unitary component of complementary series. This UIR was actually studied originally in [64].

The generic landscape of the scalar field is summarised in figure 4 .

\section{B Classification of $\mathfrak{s o}(2, d)$ unitary irreducible representations \& their characters}

We will focus on highest-weight representations, which are the physically most relevant ones. Indeed, their energy spectrum is, by construction, bounded from above or below. ${ }^{16}$ They are characterised by a highest-weight: $\lambda=(\Delta, \vec{s})$ with $\Delta$ the conformal weight, or minimal energy in $\operatorname{AdS}_{d+1}$, and $\vec{s}=\left(s_{1}, s_{2}, \ldots, s_{r}\right)$ a $\mathfrak{s o}(d)$ highest-weight labeling the spin of the representation. The highest-weight UIRs of $\mathfrak{s o}(2, d)$ can be described fields on $\mathrm{AdS}_{d+1}$ and classified as follows:

\footnotetext{
${ }^{16}$ However, remember that there are no such representations for the de Sitter case since there is no global timelike Killing vector field on de Sitter spacetime.
} 


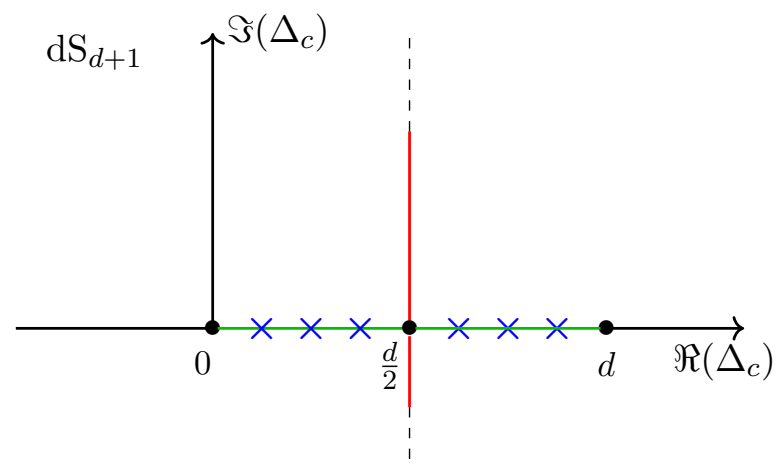

Figure 4. Repartition of the massive scalar fields in $\mathrm{dS}_{d+1}$ discussed above, as a function of the conformal weight $\Delta_{c}$. Massive field in the principal and complementary series are depicted respectively by a red and a green line, whereas the blue dots indicate a discrete collection of representations in the complementary series with $\Delta_{c}=\frac{d}{2} \pm \ell$ that would correspond to higher order singletons and their shadows in $\mathrm{AdS}_{d+1}$.

- Massive representations: $\Delta>s_{1}+d-h_{1}-1$ with $s_{1}=\cdots=s_{h_{1}}>s_{h_{1}+1}$, or $\Delta>\frac{d-2}{2}$ for $\vec{s}=\overrightarrow{0}$ and $\Delta>\frac{d-1}{2}$ for $\vec{s}=\left(\frac{1}{2}, \cdots, \frac{1}{2}\right)$, whose character reads:

$$
\chi_{[\Delta ; \vec{s}]}^{\operatorname{AdS}}(q, \vec{x})=q^{\Delta} \chi_{\vec{s}}^{\mathfrak{s o}(d)}(\vec{x}) \mathcal{P}^{(d)}(q, \vec{x})
$$

with $\mathcal{P}^{(d)}(q, \vec{x})$ given by $(2.7)$.

- Massless representations: $\Delta=\Delta_{s, h_{1}}:=s+d-h_{1}-1$, with $\vec{s}$ such that $s_{1}=$ $\cdots=s_{h_{1}} \equiv s>s_{h_{1}+1} \geqslant \cdots \geqslant\left|s_{r}\right|$, analysed in [8,9] and whose character is given by [69]:

$$
\chi_{\left[\Delta_{s, h_{1}} ; \vec{s}\right]}^{\operatorname{AdS}}(q, \vec{x})=q^{\Delta_{s, h_{1}}}\left(\chi_{\vec{s}}^{\mathfrak{s o}(d)}(\vec{x})+\sum_{k=1}^{h_{1}}(-q)^{h_{1}+1-k} \chi_{\vec{s}_{k}}^{\mathfrak{s o}(d)}(\vec{x})\right) \mathcal{P}^{(d)}(q, \vec{x})
$$

with $\vec{s}_{k}=\left(s, \ldots, s, s \frac{\uparrow}{-1}, s-1, \ldots, s \underset{\uparrow}{-1}, s_{h_{1}+1}, \ldots, s_{r}\right)$

$$
k \text { th entry } \quad h_{1} \text { th entry }
$$

Example 3. Usually, totally symmetric massless fields are considered, i.e. massless fields with $\Delta=\Delta_{s}:=s+d-2$ and $\vec{s}=(s, 0, \ldots, 0)$ for $s \in 1+\mathbb{N}$ or $\vec{s}=\left(s, \frac{1}{2}, \ldots, \frac{1}{2}\right)$ for $s \in \frac{1}{2}+\mathbb{N}$, that we will both denote $(s)$. Accordingly, their character is given by the above formula in the special case $h_{1}=1$ :

$$
\chi_{\left[\Delta_{s} ;(s)\right]}^{\operatorname{AdS}}(q, \vec{x})=q^{\Delta_{s}}\left(\chi_{(s)}^{\mathfrak{s o}(d)}(\vec{x})-q \chi_{(s-1)}^{\mathfrak{s o}(d)}(\vec{x})\right) \mathcal{P}^{(d)}(q, \vec{x})
$$

Another class of physically interesting representations (although not unitary on AdS, contrarily to the above irreps) are the so-called (totally symmetric) partially-massless fields of spin $s$ and depth $t$, with $\Delta=\Delta_{s}^{(t)}:=s+d-t-1$ and $s \geqslant t>1$. Their character reads:

$$
\chi_{\left[\Delta_{s}^{(t)} ;(s)\right]}^{\operatorname{AdS}}(q, \vec{x})=q^{\Delta_{s}^{(t)}}\left(\chi_{(s)}^{\mathfrak{s o}(d)}(\vec{x})-q^{t} \chi_{(s-t)}^{\mathfrak{s o}(d)}(\vec{x})\right) \mathcal{P}^{(d)}(q, \vec{x})
$$




\section{Verma interlude}

In this section, we recall some basic definitions on Verma module and generalised Verma modules, as well as BGG resolutions in both context. Once again, we make no attempt at full mathematical rigor, but hope to give an intuitive picture of these concept to the unfamiliar reader (for more details, see for instance [70]).

\section{C.1 Verma module}

Definition C.1 (Verma module). Let $\mathfrak{g}$ be a semisimple Lie algebra, with Cartan subalgebra $\mathfrak{h} \subset \mathfrak{g}, \Delta$ (resp. $\Delta_{ \pm} \subset \Delta$ ) its root (resp. positive/negative root) system, $\mathfrak{g}_{ \pm}$the subalgebra dual to the positive/negative root system $\Delta_{ \pm}$and $\mathfrak{b}:=\mathfrak{h} \oplus \mathfrak{g}_{+}$its Borel subalgebra. Furthermore, let $\mathcal{U}(\mathfrak{g})$ denote the universal enveloping algebra of $\mathfrak{g}$ and $\boldsymbol{\lambda} \in \Delta$ denote a weight. Let $v_{\boldsymbol{\lambda}}$ be a one-dimensional $\mathfrak{b}$-module, then the Verma module $\mathcal{V}_{\boldsymbol{\lambda}}$ is defined as:

$$
\mathcal{V}_{\boldsymbol{\lambda}}:=\mathcal{U}(\mathfrak{g}) \otimes_{\mathcal{U}(\mathfrak{b})} v_{\boldsymbol{\lambda}} \cong \mathcal{U}\left(\mathfrak{g}_{-}\right) \otimes v_{\boldsymbol{\lambda}}
$$

In other words, a Verma module is a representation space of $\mathfrak{g}$ constructed from a highest-weight vector, i.e. an eigenvector of the Cartan subalgebra which is annihilated by all raising operators. In the language of the above definition, this highest-weight vector is a one-dimensional representation of the Borel subalgebra, which is composed of the Cartan subalgebra and the subalgebra spanned by raising operators, as they have a definite action on it. In turn, any elements of $\mathcal{V}_{\boldsymbol{\lambda}}$ is of the form $\prod_{\alpha \in \Delta_{-}}\left(E_{\alpha}\right)^{n_{\alpha}} v_{\boldsymbol{\lambda}}, n_{\alpha} \in \mathbb{N}$ where $E_{\alpha}$ is a lowering operator associated to the negative root $\alpha$.

The BGG theorem for Verma modules gives a criterion for a Verma module to contain a submodule, namely it gives a condition on the highest-weight defining a submodule in a given Verma module for it to be a proper submodule. This criterion is given in the following theorem:

Theorem C.1 (Bernstein-Gel'fand-Gel'fand). Let $\mathfrak{g}$ be a Lie algebra and $\mathcal{V}_{\boldsymbol{\mu}}$ a Verma $\mathfrak{g}$-module with highest-weight $\boldsymbol{\mu}$, then the two following statements are equivalent:

- $\mathcal{V}_{\mu} \subset \mathcal{V}_{\lambda}$

- $\exists \alpha_{1}, \ldots, \alpha_{n} \in \Delta_{+}$such that $\boldsymbol{\mu}=w_{\alpha_{n}} \ldots w_{\alpha_{1}} \cdot \boldsymbol{\lambda}$ and

$$
\left(\alpha_{k}^{\vee}, w_{\alpha_{k-1}} \ldots w_{\alpha_{1}}(\boldsymbol{\lambda}+\rho)\right) \in \mathbb{N}, \quad \forall k \in\{1,2, \cdots, n\} .
$$

In the above definition, $w_{\alpha} \cdot \boldsymbol{\lambda}$ denotes the affine action of an element of the Weyl group of $\mathfrak{g}$ associated to a root $\alpha \in \Delta$, i.e. $w_{\alpha} \cdot \boldsymbol{\lambda}:=\boldsymbol{\lambda}-\alpha\left(\alpha^{\vee}, \boldsymbol{\lambda}+\rho\right)=w_{\alpha}(\boldsymbol{\lambda}+\rho)-\rho, \alpha^{\vee}:=\frac{2 \alpha}{(\alpha, \alpha)}$, where $(\cdot, \cdot)$ denotes the Killing form on $\mathfrak{g}$, and $\rho$ its Weyl vector.

Example 4. Let us consider a very simple case to illustrate this theorem. Taking $\mathfrak{s l}(2) \cong$ $\mathfrak{s o}(3)$ and a Verma module based on the highest-weight $\lambda=s \in \frac{1}{2} \mathbb{N}$, we can start looking for submodules using the BGG theorem. The Weyl group of $\mathfrak{s o}(3)$ is $\mathbb{Z}_{2}$ and its Weyl vector $\rho=\frac{1}{2}$. As a consequence, the only non trivial element of the Weyl group, that we will note $w$, is the one flipping the sign of the weight on which it will act and is associated to the 
positive root (the unit basis vector of the root space, as $\mathfrak{s o}(3)$ is of rank 1 , that we do not bother writing), and therefore the only weight that can be obtained from an action of the Weyl group on $\lambda$ is $w \cdot \lambda=\lambda-2(\lambda+\rho)=-(\lambda+1)$. Having looked at a particularly simple case of rank 1, the relevance of the criterion (C.2) cannot be fully seen, however, we were able to recover the standard result that $J_{+}\left(J_{-}\right)^{s+1} v_{\lambda}=0$ (where $v_{\lambda}$ is the highest-weight vector defining the Verma module), i.e. $\left(J_{-}\right)^{s+1} v_{\lambda}$ defines a submodule that needs to be modded out in order to obtain an irreducible representation of $\mathfrak{s o}(3)$, without having to compute explicitly the action of the ladder operators.

Recall that an integral dominant weight $\boldsymbol{\lambda}$ is defined to be a weight such that, for all positive root $\alpha \in \Delta_{+}$, it verifies $\left(\boldsymbol{\lambda}, \alpha^{\vee}\right) \in \mathbb{N}$. An important property for us is that for integral dominant weights, every element of the Weyl group verifies the condition (C.2).

Finally, we need to introduce the notion of length of a Weyl group element, in order to define the BGG resolution of an irreducible Verma module.

Definition C.2 (Length of a Weyl group element). Let $\mathfrak{g}$ be a Lie algebra, and $w \in \mathcal{W}$ an element of its Weyl group. The length of $w$, noted $\ell(w)$ is defined to be the minimal number of reflections $w_{\alpha^{(i)}}$ associated to simple root $\alpha^{(i)}$ such that $w$ is given as a product

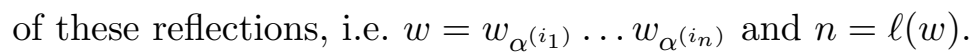

Theorem C.2 (Bernstein-Gel'fand-Gel'fand resolution). Let $\boldsymbol{\lambda}$ be an integral dominant highest-weight and $\mathcal{D}_{\boldsymbol{\lambda}}$ the corresponding finite-dimensional and irreducible highest-weight module. There exists a long exact sequence:

$$
0 \rightarrow \bigoplus_{w \in \mathcal{W}, \ell(w)=n} \mathcal{V}_{w \cdot \boldsymbol{\lambda}} \rightarrow \cdots \rightarrow \bigoplus_{w \in \mathcal{W}, \ell(w)=1} \mathcal{V}_{w \cdot \boldsymbol{\lambda}} \rightarrow \mathcal{V}_{\boldsymbol{\lambda}} \rightarrow \mathcal{D}_{\boldsymbol{\lambda}} \rightarrow 0
$$

where $n$ is the maximal length of elements of $\mathcal{W}$.

\section{C.2 Generalised Verma module}

Now we can turn to the case of a generalised Verma module, which is the one relevant for this paper, and we start by recalling the definition of such modules:

Definition C.3 (Generalised Verma module). Let $\mathfrak{g}$ be a finite dimensional Lie algebra, $\mathfrak{p}$ a parabolic subalgebra and $\mathbb{V}_{\boldsymbol{\lambda}}$ a finite dimensional representation space of $\mathfrak{p}$ with highestweight $\boldsymbol{\lambda}$. Then the generalised Verma module $\mathcal{V}_{\boldsymbol{\lambda}}$ is defined as:

$$
\mathcal{V}_{\boldsymbol{\lambda}}:=\mathcal{U}(\mathfrak{g}) \otimes_{\mathcal{U}(\mathfrak{p})} \mathbb{V}_{\boldsymbol{\lambda}}
$$

The BGG resolution for generalised Verma modules is then quite similar to the one previously exposed for Verma modules. The main difference comes from a decomposition of the Weyl group induced by the choice of a parabolic subalgebra. A convenient way to parametrise such a subalgebra is to choose a subset $\Delta_{\mathfrak{p}}$ of the root space of $\mathfrak{g}: \Delta_{\mathfrak{p}}:=$ span $\left\{\alpha^{(i)} \in \Delta^{(s)} \mid i=1, \ldots, m ; m \leqslant r\right\}$, with $\Delta^{(s)}$ the set of simple roots and $r$ the rank of $\mathfrak{g}$. Then one can define the subalgebra $\overline{\mathfrak{g}}$ dual to subspace of the weight space generated by $\Delta_{\mathfrak{p}}$ and decompose it as $\overline{\mathfrak{g}}:=\overline{\mathfrak{h}} \bigoplus \mathfrak{g}^{ \pm \alpha^{(i)}}$, where $\overline{\mathfrak{h}}$ and $\mathfrak{g}^{ \pm \alpha^{(i)}}$ are the space spanned $\alpha^{(i)} \in \Delta_{\mathfrak{p}}$ 
by, respectively, the Cartan generators and ladder operators associated to the roots in $\Delta_{\mathfrak{p}}$. Then, the parabolic subalgebra defined by the choice of $\Delta_{\mathfrak{p}}$ is the subalgebra generated by $\overline{\mathfrak{g}}$ and the full Cartan subalgebra of $\mathfrak{h}$ of $\mathfrak{g}$ together with all the positive ladder operators of $\mathfrak{g}$. In other words, the parabolic subalgebra $\mathfrak{p}$ is the Borel subalgebra augmented with a part of the negative ladder operators so that this set of generators spans a proper subalgebra of $\mathfrak{g}$.

With this decomposition at hand, one can define the subgroup $\overline{\mathcal{W}}$ of the Weyl group of $\mathfrak{g}$ generated by reflections associated to the subset of simple roots of $\Delta_{\mathfrak{p}}$. This subgroup corresponds to the Weyl group of $\overline{\mathfrak{g}}$. Another subgroup $\mathcal{W}^{\prime}$ is the one composed of elements of $\mathcal{W}$ such that any of the positive roots of $\Delta_{\mathfrak{p}}$ can be obtained by applying a element of $\mathcal{W}^{\prime}$ to a positive root in $\Delta_{+}: \mathcal{W}^{\prime}:=\left\{w \in \mathcal{W} \mid \Delta_{\mathfrak{p}}^{(+)} \subset w \Delta_{+}\right\}$. A property is that every element of the full Weyl group $\mathcal{W}$ can be decomposed as a product of elements of those two subgroups: $\forall w \in \mathcal{W}, \exists \bar{w} \in \overline{\mathcal{W}}, w^{\prime} \in \mathcal{W}^{\prime}$ such that $w=\bar{w} w^{\prime}$.

The BGG resolution for a generalised Verma module with highest-weight $\boldsymbol{\lambda}$ being an integral dominant weight, is defined almost as in the case of Verma module, except for the fact the the full Weyl group should be substituted with the subgroup $\mathcal{W}^{\prime}$ : the long exact sequence is

$$
0 \rightarrow \bigoplus_{w \in \mathcal{W}^{\prime}, \ell(w)=n} \mathcal{V}_{w \cdot \boldsymbol{\lambda}} \rightarrow \cdots \rightarrow \bigoplus_{w \in \mathcal{W}^{\prime}, \ell(w)=1} \mathcal{V}_{w \cdot \boldsymbol{\lambda}} \rightarrow \mathcal{V}_{\boldsymbol{\lambda}} \rightarrow \mathcal{D}_{\boldsymbol{\lambda}} \rightarrow 0
$$

\section{Characters from Bernstein-Gel'fand-Gel'fand resolutions}

In [39], the structure of $\mathfrak{s o}^{*}(2+d)$ modules (where $*$ denotes the complexified algebra) was spelled out using BGG resolutions for generalised Verma modules (see [31, 38] for earlier similar results at the group level). On the representation theory side (as recalled in appendix C), they consist of a series of homomorphisms between generalised Verma modules, induced by particular elements of the Weyl group and such that the module in the image of each of these maps is a submodule of the previous one.

Let us also introduce the following notations:

- A height- $p$ Young diagram, with $p \leqslant r$ will be denoted:

$$
\mathbb{Y}_{p}:=\left(s_{1}, s_{2}, \ldots, \underset{\substack{\uparrow \\ p \text { th }}}{\left.s_{p}, 0, \ldots, 0\right)=\vec{s}}\right.
$$

- An important operation on Young diagrams when dealing with the exceptional series is to remove one row from it and to delete one box in each of the following rows (i.e. situated below the one that was just removed). We will denote the diagram obtained from $\mathbb{Y}_{p}$ after having performed the above modifications as:

$$
\check{\mathbb{Y}}_{p}^{(i)}:=\left(s_{1}, \ldots, s_{i-1}, s_{i+1}-1, \ldots, \underset{\substack{\uparrow \\ i-\text { th }}}{(p-1)-\text { th }} s_{p}-1,0, \ldots, 0\right)
$$

- A generalised Verma module based on the $\mathfrak{s o}(2) \oplus \mathfrak{s o}(d)$ highest-weight $\boldsymbol{\lambda}$ will generically be denoted $\mathcal{V}_{\boldsymbol{\lambda}}$, except when it is irreducible in which case we will write $\mathcal{D}_{\boldsymbol{\lambda}}$. The 
translation rule from this $\mathfrak{s o}(2) \oplus \mathfrak{s o}(d)$ highest-weight to the conformal-weight/lowestenergy $\Delta$ and the $\mathfrak{s o}(d)$ highest-weight $\vec{s}$ is:

$$
[\Delta ; \vec{s}]=\boldsymbol{\lambda}=\left(\lambda_{0}, \lambda_{1}, \cdots, \lambda_{r}\right)=\left(-\Delta, s_{1}, \cdots, s_{r}\right)
$$

- Finally, our elementary building block in writing characters for $\mathfrak{s o}^{*}(2+d)$ are the characters of irreducible $\mathfrak{s o}(2) \oplus \mathfrak{s o}(d)$ modules. We will introduce for them the notation:

$$
\mathcal{Y}_{[\Delta ; \vec{s}]}(q, \vec{x})=q^{\Delta} \chi_{\vec{s}}^{\mathfrak{s o}(d)}(\vec{x}) .
$$

The character of the generalised Verma module induced by the irrep $[\Delta ; \vec{s}]$ of $\mathfrak{s o}(2) \oplus$ $\mathfrak{s o}(d)$ is

$$
\mathcal{Y}_{[\Delta ; \vec{s}]}(q, \vec{x}) \mathcal{P}^{(d)}(q, \vec{x}),
$$

with $\mathcal{P}^{(d)}(q, \vec{x})$ given by $(2.7)$.

For the sake of self-containedness, for each relevant case we summarise the results of [39] on generalised Verma modules and then deduce the corresponding character:

Odd $\boldsymbol{d}=\mathbf{2} \boldsymbol{r}+\mathbf{1}$. Defining the sequence of $\mathfrak{s o}^{*}(2+d)$ weights, where the first entry is the $\mathfrak{s o}(2)$ weight and the $r$ following entries are the components of the $\mathfrak{s o}(d)$ highest-weight:

$$
\begin{array}{rlr}
(\lambda)_{N} & =\left(\lambda_{N}-N, \lambda_{0}+1, \ldots, \lambda_{N-1}+1, \lambda_{N+1}, \ldots, \lambda_{r}\right), & (N=0, \ldots, r), \\
(\lambda)_{K+r} & =\left(-\lambda_{r+1-K}-K-r, \lambda_{0}+1, \ldots, \lambda_{r-K}+1, \lambda_{r+2-K}, \ldots, \lambda_{r}\right), & (K=1, \ldots, r), \\
(\lambda)_{2 r+1} & =\left(-\lambda_{0}-2 r-1, \lambda_{1}, \ldots, \lambda_{r}\right), &
\end{array}
$$

the following sequence is exact:

$$
0 \rightarrow \mathcal{V}_{(\lambda)_{2 r+1}} \rightarrow \mathcal{V}_{(\lambda)_{2 r}} \rightarrow \cdots \rightarrow \mathcal{V}_{(\lambda)_{1}} \rightarrow \mathcal{V}_{(\lambda)_{0}} \rightarrow 0
$$

It can be shown that in odd dimensions, no subsingular module can arise. In other words, the above exact sequence implies the following short exact sequences:

$$
0 \rightarrow \mathcal{V}_{(\lambda)_{2 r}} \rightarrow \mathcal{D}_{(\lambda)_{2 r+1}} \rightarrow 0
$$

and

$$
0 \rightarrow \mathcal{D}_{(\lambda)_{N+1}} \rightarrow \mathcal{V}_{(\lambda)_{N}} \rightarrow \mathcal{D}_{(\lambda)_{N}} \rightarrow 0, \quad(N=0, \ldots, 2 r)
$$

This implies that the irreducible highest-weight module in the above sequence with weight $(\lambda)_{N}$ is given by the quotient:

$$
\mathcal{D}_{(\lambda)_{N}}=\frac{\mathcal{V}_{(\lambda)_{N}}}{\mathcal{D}_{(\lambda)_{N+1}}}
$$

At the character level, this translates as:

$$
\begin{aligned}
\chi_{(\lambda)_{N}}(q, \vec{x}) & =\mathcal{Y}_{(\lambda)_{N}}(q, \vec{x}) \mathcal{P}^{(d)}(q, \vec{x})-\chi_{(\lambda)_{N+1}}(q, \vec{x}) \\
& =\sum_{k=0}^{2 r+1-N}(-1)^{k} \mathcal{Y}_{(\lambda)_{N+k}}(q, \vec{x}) \mathcal{P}^{(d)}(q, \vec{x})
\end{aligned}
$$


Even $\boldsymbol{d}=\mathbf{2} \boldsymbol{r}$. The sequence of weights is modified in this case:

$$
\left\{\begin{aligned}
(\lambda)_{-k} & =\left(\lambda_{r-k}-r+k, \lambda_{0}+1, \ldots, \lambda_{r-k-1}+1, \lambda_{r-k+1}, \ldots, \lambda_{r}\right), \quad(k=1, \ldots, r) \\
(\lambda)_{0} & =\left(\lambda_{r}-r, \lambda_{0}+1, \ldots, \lambda_{r-1}+1\right) \\
(\lambda)_{0^{\prime}} & =\left(-\lambda_{r}-r, \lambda_{0}+1, \ldots,-\lambda_{r-1}-1\right), \\
(\lambda)_{+k} & =\left(-\lambda_{r-k}-r-k, \lambda_{0}, \ldots, \lambda_{r-k-1}+1, \lambda_{r-k+1}, \ldots,-\lambda_{r}\right), \quad(k=1, \ldots, r)
\end{aligned}\right.
$$

which differs from the odd-dimensional case by the presence of non-standard (NS) homomorphisms and by a rhombus ${ }^{17}$ in the middle of the sequence, yielding:

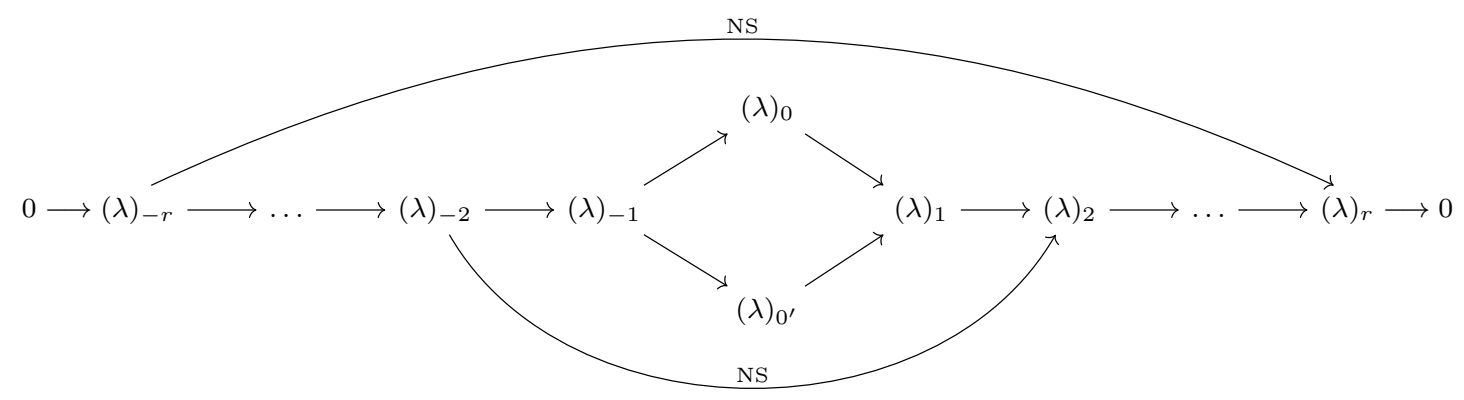

The main difference with the odd-dimensional case is the possibility of subsingular modules, but no subsubsingular ones. The above sequence leads to the following short exact sequences:

$$
\left\{\begin{array}{rl}
0 & \rightarrow \mathcal{U}_{(\lambda)_{N+1}} \rightarrow \mathcal{V}_{(\lambda)_{N}} \rightarrow \mathcal{D}_{(\lambda)_{N}} \rightarrow 0, \\
0 \rightarrow \mathcal{V}_{(\lambda)_{-N}}^{*} & \rightarrow \mathcal{U}_{(\lambda)_{N+1}} \rightarrow \mathcal{D}_{(\lambda)_{N+1}} \rightarrow 0
\end{array} \quad(N=-1, \ldots,-r),\right.
$$

together with

$$
0 \rightarrow \mathcal{D}_{(\lambda)_{N+1}} \rightarrow \mathcal{V}_{(\lambda)_{N}} \rightarrow \mathcal{D}_{(\lambda)_{N}} \rightarrow 0, \quad(N=0, \ldots, r)
$$

and

$$
0 \rightarrow \mathcal{V}_{(\lambda)_{1}}^{*} \rightarrow \mathcal{U}_{(\lambda)_{0}} \rightarrow \mathcal{D}_{(\lambda)_{0}} \oplus \mathcal{D}_{(\lambda)_{0^{\prime}}} \rightarrow 0,
$$

where $\mathcal{V}_{(\lambda)}^{*}$ denotes the contragradient module. The sequence (D.15) expresses the irreducible module $\mathcal{D}_{(\lambda)_{N}}$ for $N=-1, \ldots,-r+1$ as two different quotients:

$$
\mathcal{D}_{(\lambda)_{N}}=\frac{\mathcal{V}_{(\lambda)_{N}}}{\mathcal{U}_{(\lambda)_{N+1}}}=\frac{\mathcal{U}_{(\lambda)_{N}}}{\mathcal{V}_{(\lambda)_{-N+1}}^{*}} \quad(N=-1, \ldots,-r+1)
$$

which can be translated into characters, yielding:

$$
\mathcal{Y}_{(\lambda)_{N}}(q, \vec{x}) \mathcal{P}^{(d)}(q, \vec{x})-\mathcal{C}_{(\lambda)_{N+1}}(q, \vec{x})=\mathcal{C}_{(\lambda)_{N}}(q, \vec{x})-\mathcal{Y}_{(\lambda)_{-N+1}}(q, \vec{x}) \mathcal{P}^{(d)}(q, \vec{x})
$$

\footnotetext{
${ }^{17}$ The appearence of this rhombus is due to the fact that there exist two elements of the Weyl group $\mathcal{W}^{\prime}$ with the same length for $d=2 r$. This can be seen in (A.16) of [39].
} 
where $\mathcal{C}_{(\lambda)_{N}}$ is the character of the reducible module $\mathcal{U}_{(\lambda)_{N}}$. This can be used to compute $\mathcal{C}_{(\lambda)_{-k}}(k=1, \ldots, r-1)$ :

$$
\begin{aligned}
\mathcal{C}_{(\lambda)_{-k}}(q, \vec{x})=\left(\mathcal{Y}_{(\lambda)_{-k}}(q, \vec{x})+\mathcal{Y}_{(\lambda)_{k+1}}(q, \vec{x})\right) \mathcal{P}^{(d)}(q, \vec{x})-\mathcal{C}_{(\lambda)_{-k+1}}(q, \vec{x}) \\
=\sum_{n=0}^{k-1}(-)^{n}\left(\mathcal{Y}_{(\lambda)_{-k+n}}(q, \vec{x})+\mathcal{Y}_{(\lambda)_{k+1-n}}(q, \vec{x})\right) \mathcal{P}^{(d)}(q, \vec{x})+(-)^{k} \mathcal{C}_{(\lambda)_{0}}(q, \vec{x}) .
\end{aligned}
$$

Using (D.17), we can express $\mathcal{C}_{(\lambda)_{0}}(q, \vec{x})$ as:

$$
\mathcal{C}_{(\lambda)_{0}}(q, \vec{x})=\chi_{(\lambda)_{0}}(q, \vec{x})+\chi_{(\lambda)_{0^{\prime}}}(q, \vec{x})+\mathcal{Y}_{(\lambda)_{1}}(q, \vec{x}) \mathcal{P}^{(d)}(q, \vec{x})
$$

Now as both modules $\mathcal{D}_{(\lambda)_{0}}$ and $\mathcal{D}_{(\lambda)_{0^{\prime}}}$ are resolved by the same short sequence as in the odd-dimensional case, there character can be straightforwardly computed:

$$
\chi_{(\lambda)_{0}}(q, \vec{x})=\sum_{N=0}^{r}(-)^{N} \mathcal{Y}_{(\lambda)_{N}}(q, \vec{x}) \mathcal{P}^{(d)}(q, \vec{x}),
$$

idem for $\chi_{(\lambda)_{0^{\prime}}}(q, \vec{x})$ with the sum starting at $N=0^{\prime}$. Plugging this back into (D.21), we finally obtain the explicit expression of $\mathcal{C}_{(\lambda)_{-k}}$ in terms of the factors $\mathcal{Y}_{(\lambda)_{N}}$ and $\mathcal{P}^{(d)}$. This formula can then be used to express the character of the irreducible module $\mathcal{D}_{(\lambda)_{-k}}$ :

$$
\begin{aligned}
\chi_{(\lambda)_{-k}}(q, \vec{x})= & \mathcal{Y}_{(\lambda)_{-k}}(q, \vec{x}) \mathcal{P}^{(d)}(q, \vec{x})-\mathcal{C}_{(\lambda)_{-k+1}}(q, \vec{x}) \\
= & \sum_{n=0}^{k}(-)^{k+n}\left(\mathcal{Y}_{(\lambda)_{n}}(q, \vec{x})+\mathcal{Y}_{(\lambda)_{-n}}(q, \vec{x})\right) \mathcal{P}^{(d)}(q, \vec{x}) \\
& +2 \sum_{n=k+1}^{r}(-)^{k+n} \mathcal{Y}_{(\lambda)_{n}}(q, \vec{x}) \mathcal{P}^{(d)}(q, \vec{x})
\end{aligned}
$$

where the term $\mathcal{Y}_{(\lambda)_{-n}}$ for $n=0$ has to be understood as $\mathcal{Y}_{(\lambda)_{0^{\prime}}}$, and the last sum is absent when $k=r$.

Identifying the exceptional series. Starting from:

$$
\left(\lambda_{0}, \lambda_{1}, \ldots, \lambda_{r}\right)=\left(s_{1}-1, s_{2}-1, \ldots, s_{p}-1,0, \ldots, 0\right)=\left(s_{1}-1, \check{Y}_{p}^{(1)}\right)
$$

as the weight of the long exact sequence, then the different weights enumerated above for $d=2 r+1$ take the form:

$$
(\lambda)_{N}=\left\{\begin{array}{lr}
\left(s_{N+1}-(N+1), \check{\mathbb{Y}}_{p}^{(N+1)}\right), & 0 \leqslant N \leqslant p-1 \\
\left(-N, \mathbb{Y}_{p}, \mathbf{1}^{N-p}\right), & p \leqslant N \leqslant r \\
\left(-N, \mathbb{Y}_{p}, \mathbf{1}^{d-p-N}\right), & r+1 \leqslant N \leqslant d-p \\
\left(-\left(s_{d+1-N}+N+1\right), \check{\mathbb{Y}}_{p}^{(d+1-N)}\right), & d+1-p \leqslant N \leqslant d
\end{array}\right.
$$


Now let us show that the character of the module at level $N=p$ reproduces the formula of characters for the exceptional series in [36]:

$$
\begin{aligned}
& \chi_{(\lambda)_{p}}(q, \vec{x})=\sum_{k=p}^{2 r+1}(-1)^{p+k} \mathcal{Y}_{(\lambda)_{k}}(q, \vec{x}) \mathcal{P}^{(d)}(q, \vec{x}) \\
& =\sum_{k=p}^{r}(-1)^{k+p} q^{k} \chi_{\left(\mathbb{Y}_{p}, \mathbf{1}^{k-p}\right)}^{\mathfrak{s o}(d)}(\vec{x}) \mathcal{P}^{(d)}(q, \vec{x})+\sum_{k^{\prime}=r+1}^{d-p}(-1)^{k^{\prime}+p} q^{k^{\prime}} \chi_{\left(\mathbb{Y}_{p}, \mathbf{1}^{d-p-k^{\prime}}\right)}^{\mathfrak{s o}(\vec{x})} \mathcal{P}^{(d)}(q, \vec{x}) \\
& +\sum_{k^{\prime \prime}=d+1-p}^{d}(-1)^{k^{\prime \prime}+p} q^{s_{d+1-k^{\prime \prime}+k^{\prime \prime}-1}} \chi_{\check{\mathbb{Y}}_{p}^{\mathfrak{s o}(d)}\left(d+1-k^{\prime \prime}\right)}(\vec{x}) \mathcal{P}^{(d)}(q, \vec{x}) \\
& =\sum_{i=1}^{r-p}(-1)^{i} q^{p+i} \chi_{\left(\mathbb{Y}_{p}, \mathbf{1}^{i}\right)}^{\mathfrak{s o}(d)}(\vec{x}) \mathcal{P}^{(d)}(q, \vec{x})+\sum_{j=1}^{r-p} \underbrace{(-1)^{d+j}}_{=-(-1)^{j}} q^{d-p-j} \chi_{\left(\mathbb{Y}_{p}, \mathbf{1}^{j}\right)}^{\mathfrak{s o}(d)}(\vec{x}) \mathcal{P}^{(d)}(q, \vec{x}) \\
& +\left(q^{p} \chi_{\mathbb{Y}_{p}}^{\mathfrak{s o}(d)}(\vec{x})-q^{d-p} \chi_{\mathbb{Y}_{p}}^{\mathfrak{s o}(d)}(\vec{x})\right) \mathcal{P}^{(d)}(q, \vec{x})+\sum_{\ell=1}^{p} \underbrace{(-1)^{d+1+h+\ell}}_{=-(-1)^{p+1+\ell}} q^{s_{\ell}+d-\ell} \chi_{\dot{\mathbb{Y}}_{p}^{(\ell)}}^{\mathfrak{s o}(d)}(\vec{x}) \mathcal{P}^{(d)}(q, \vec{x}) \\
& =\left(q^{p}-q^{d-p}\right) \chi_{\mathbb{Y}_{p}}^{\mathfrak{s o}(d)}(\vec{x}) \mathcal{P}^{(d)}(q, \vec{x})-\sum_{\ell=1}^{p}(-1)^{p+1+\ell} q^{s_{\ell}+d-\ell} \chi_{\overleftrightarrow{\mathbb{Y}}_{p}^{(\ell)}}^{\mathfrak{s o}(d)}(\vec{x}) \mathcal{P}^{(d)}(q, \vec{x}) \\
& +\sum_{m=1}^{r-p}(-1)^{m}\left(q^{p+m}-q^{d-p-m}\right) \chi_{\left(\mathbb{Y}_{p}, \mathbf{1}^{m}\right)}^{\mathfrak{s o}(d)}(\vec{x}) \mathcal{P}^{(d)}(q, \vec{x})
\end{aligned}
$$

where we used $i=k-p, j=d-p-k^{\prime}$ and $\ell=d+1-k^{\prime \prime}$ when going from the second to the third equality.

Turning to the $d=2 r$ case, we now have the following series of weights:

$$
\left\{\begin{array}{rlrl}
(\lambda)_{-n} & =\left(s_{r-n+1}-1-(r-n), \check{\mathbb{Y}}_{p}^{(r-n+1)}\right), & n=r, \ldots, r-p+1 \\
(\lambda)_{-n} & =\left(-(r-n), \mathbb{Y}_{h}, \mathbf{1}^{r-n-p}\right), & n & =r-p, \ldots, 1 \\
(\lambda)_{0} & =\left(-r, \mathbb{Y}_{p}, \mathbf{1}_{+}^{r-p}\right), & (\lambda)_{0^{\prime}} & =\left(-r, \mathbb{Y}_{p}, \mathbf{1}_{-}^{r-p}\right) \\
(\lambda)_{n} & =\left(-(r+n), \mathbb{Y}_{p}, \mathbf{1}^{r-n-p}\right), & n & =1, \ldots, r-p \\
(\lambda)_{n} & =\left(-s_{r-n+1}-(r+n)+1, \check{Y}_{p}^{(r-n+1)}\right), & & n=r-p+1, \ldots, r
\end{array}\right.
$$

where $\mathbf{1}_{ \pm}^{m}$ denote the $m$ last components of the $\mathfrak{s o}(2 r)$ weight, these components all being egal to 1 except for the last one which can be \pm 1 .

Using (D.24), we can write the character of the irreducible module corresponding to the highest-weight $(\lambda)_{-(r-p)}$ which we identified as the character of the exceptional series 
in odd spacetime dimension:

$$
\begin{aligned}
\chi_{(\lambda)_{-(r-p)}(q, \vec{x})=} \sum_{n=0}^{r-p}(-)^{r-p+n}\left(\mathcal{Y}_{(\lambda)_{n}}(q, \vec{x})+\mathcal{Y}_{(\lambda)_{-n}}(q, \vec{x})\right) \mathcal{P}^{(d)}(q, \vec{x}) \\
+2 \sum_{n=r-p+1}^{r}(-)^{r-p+n} \mathcal{Y}_{(\lambda)_{n}}(q, \vec{x}) \mathcal{P}^{(d)}(q, \vec{x}) \\
=\sum_{n=1}^{r-p}(-)^{r-p+n}\left(q^{r+n}+q^{r-n}\right) \chi_{\left(\mathbb{Y}_{p}, \mathbf{1}^{r-p-n}\right)}^{\mathfrak{s o}(d)}(\vec{x}) \mathcal{P}^{(d)}(q, \vec{x}) \\
\quad+(-)^{r-p} q^{r}\left(\chi_{\left(\mathbb{Y}_{p}, \mathbf{1}_{+}^{r-p}\right)}^{\mathfrak{s o}(d)}(\vec{x})+\chi_{\left(\mathbb{Y}_{p}, \mathbf{1}_{-}^{r-p}\right)}^{\mathfrak{s o}(d)}(\vec{x})\right) \mathcal{P}^{(d)}(q, \vec{x}) \\
\quad+2 \sum_{n=r-p+1}^{r}(-)^{r-p+n} q^{s_{r-n+1}+r+n-1} \chi_{\mathbb{\mathbb { Y }}_{p}^{\mathfrak{s o}(d)}(r+1)}(\vec{x}) \mathcal{P}^{(d)}(q, \vec{x}) \\
=\sum_{n=0}^{r-p-1}(-)^{n}\left(q^{d-p-n}+q^{p+n}\right) \chi_{\left(\mathbb{Y}_{p}, \mathbf{1}^{n}\right)}^{\mathfrak{s o}(d)}(\vec{x}) \mathcal{P}^{(d)}(q, \vec{x}) \\
\quad+2 \sum_{\ell=1}^{h}(-)^{p+\ell+1} q^{s_{\ell}+d-\ell} \chi_{\overleftarrow{\mathbb{Y}}_{p}^{\mathfrak{s o}(\ell)}(\vec{x}) \mathcal{P}^{(d)}(q, \vec{x})} \\
\quad+(-)^{r-p} q^{d / 2}\left(\chi_{\left(\mathbb{Y}_{p}, \mathbf{1}_{+}^{r-p}\right)}^{\mathfrak{s o}(d)}(\vec{x})+\chi_{\left(\mathbb{Y}_{p}, \mathbf{1}_{-}^{r-p}\right)}^{\mathfrak{s o}(d)}(\vec{x})\right) \mathcal{P}^{(d)}(q, \vec{x})
\end{aligned}
$$

Identifying the discrete series. Starting in $d=2 r+1$ from:

$$
(\lambda)_{0}=\left(\lambda_{0}, \lambda_{1}, \ldots, \lambda_{r}\right)=\left(s_{1}-1, \mathbb{Y}_{r, k}\right),
$$

with

$$
\mathbb{Y}_{r, k}=\left(s_{2}-1, s_{3}-1, \ldots, s_{r}-1, k-1\right)
$$

leads to the following sequence of weights:

$$
(\lambda)_{r+1}=\left(-k-r, s_{1}, \ldots, s_{r}\right)
$$

and

$$
(\lambda)_{r+K}=\left(-s_{r+2-K}-r-K+1, s_{1}, \ldots, s_{r+1-K}, s_{r+3-K}-1, \ldots, s_{r}-1, k-1\right), K=2, \ldots, r+1 .
$$

It turns out that the character corresponding to the irreducible model at the level $r+1$ in this sequence, computed with (D.13), exactly reproduces the one given by Hirai in [36] for the direct sum of two discrete series representations based on the highest-weight vector 
$\vec{s}=\left(s_{1}, \ldots, s_{r}\right)$ and whose conformal weight is determined by the integer $k$ :

$$
\begin{aligned}
\chi_{(\lambda)_{r+1}}(q, \vec{x})= & \sum_{j=0}^{r}(-1)^{j} \mathcal{Y}_{(\lambda)_{r+1+j}}(q, \vec{x}) \mathcal{P}^{(d)}(q, \vec{x}) \\
= & q^{k+r} \chi_{\vec{s}}^{\mathfrak{s o}(d)}(\vec{x}) \mathcal{P}^{(d)}(q, \vec{x}) \\
& +\sum_{j=1}^{r}(-1)^{j} q^{s_{r+1-j}+r+j} \chi_{\left(s_{1}, \ldots, s_{r-j}, s_{r+2-j}-1, \ldots, s_{r}-1, k-1\right)}^{\mathfrak{s o}(d)}(\vec{x}) \mathcal{P}^{(d)}(q, \vec{x}) \\
= & q^{k+r} \chi_{\vec{s}}^{\mathfrak{s o}(d)}(\vec{x}) \mathcal{P}^{(d)}(q, \vec{x})+\sum_{i=1}^{r}(-1)^{r+1+i} q^{s_{i}+d-i} \chi_{\overleftarrow{\mathbb{Y}}_{\vec{s}, k}^{(i)}}^{\mathfrak{s o}(d)}(\vec{x}) \mathcal{P}^{(d)}(q, \vec{x})
\end{aligned}
$$

where we introduced the notation $\check{\mathbb{Y}}_{\vec{s}, k}^{(i)}=\left(s_{1}, \ldots, s_{i-1}, s_{i+1}-1, \ldots, s_{r}-1, k-1\right)$ in the last line.

\section{E Poincaré characters revisited}

As clearly recalled in $[27,71]$, the characters of the Poincaré group $\operatorname{ISO}(1, d)=\operatorname{SO}(1, d) \ltimes$ $\mathbb{R}^{d+1}$ follow from Frobenius formula for semi-direct product groups:

$$
\chi[(\Lambda, \alpha)]=\int_{\mathcal{O}_{p}} d \mu(k) \delta_{\mu}(k, \Lambda \cdot k) e^{i\langle k, \alpha\rangle} \chi_{\mathcal{R}}\left(g_{k}^{-1} \Lambda g_{k}\right)
$$

where $(\Lambda, \alpha) \in \operatorname{ISO}(1, d)$, with $\Lambda \in \operatorname{SO}(1, d)$ and $\alpha \in \mathbb{R}^{d+1}$, is a generic element of the Poincaré group. The integral (E.1) is defined over the orbit of the momentum $p \in\left(\mathbb{R}^{d+1}\right)^{*}$ :

$$
\mathcal{O}_{p}=\{\Lambda \cdot p \mid \Lambda \in \mathrm{SO}(1, d)\} \subset\left(\mathbb{R}^{d+1}\right)^{*}
$$

In the integral (E.1), the symbols $d \mu(k)$ and $\delta_{\mu}\left(k, k^{\prime}\right)$ denote, respectively, the invariant measure on $\mathcal{O}_{p}$ and the associated Dirac distribution, $\chi_{\mathcal{R}}$ is the character of an irreducible representation $\mathcal{R}$ of the little group labeled in what follows by the highest-weight $\vec{s}$, and $\langle k, \alpha\rangle:=k_{\mu} \alpha^{\mu}$. The map

$$
g: \mathcal{O}_{p} \longrightarrow \mathrm{SO}(1, d): q \longmapsto g_{q}
$$

is such that $g_{q} \cdot p=q, \forall q \in \mathcal{O}_{p}$. Notice that when integrating over the orbit $\mathcal{O}_{p}$, because of the delta function forcing $\Lambda$ to be an element of the little group of $p, g_{k}^{-1} \Lambda g_{k}$ runs through the equivalence class of such elements.

\section{E.1 Massive representations}

In this case, the orbit is $\mathcal{O}_{p}=\left\{k \in\left(\mathbb{R}^{d+1}\right)^{*} \mid-m^{2}=\eta_{\mu \nu} k^{\mu} k^{\nu}\right\}$. The corresponding little group is $\mathrm{SO}(d)$. The mass- $m$ spin- $\vec{s}$ massive UIR will be denoted $[m ; \vec{s}]$.

When $d=2 r$, we can take $\Lambda$ of the form:

$$
\Lambda=\left(\begin{array}{ccccc}
1 & 0 & \ldots & 0 & 0 \\
0 & R\left(\theta_{1}\right) & 0 & \ldots & 0 \\
\vdots & 0 & \ddots & 0 & \vdots \\
0 & \vdots & 0 & R\left(\theta_{r-1}\right) & 0 \\
0 & 0 & \ldots & 0 & R\left(\theta_{r}\right)
\end{array}\right)
$$


where the matrices $R\left(\theta_{i}\right), i=1, \ldots, r-1$ are usual $\mathrm{SO}(2)$ elements:

$$
R\left(\theta_{i}\right)=\left(\begin{array}{cc}
\cos \left(\theta_{i}\right) & -\sin \left(\theta_{i}\right) \\
\sin \left(\theta_{i}\right) & \cos \left(\theta_{i}\right)
\end{array}\right)
$$

When $d=2 r+1$ however, we will consider an element $\Lambda$ of the form:

$$
\Lambda=\left(\begin{array}{ccccc}
1 & 0 & \ldots & 0 & 0 \\
0 & R\left(\theta_{1}\right) & 0 & \ldots & 0 \\
\vdots & 0 & \ddots & 0 & \vdots \\
0 & \vdots & 0 & R\left(\theta_{r-2}\right) & 0 \\
0 & 0 & \ldots & 0 & R^{\prime}\left(\theta_{r}, \varphi\right)
\end{array}\right)
$$

with $R^{\prime}\left(\theta_{r}, \varphi\right)$ the $\mathrm{SO}(3)$ matrix:

$$
\begin{aligned}
R^{\prime}\left(\theta_{r}, \varphi\right) & =\left(\begin{array}{ccc}
\cos \left(\theta_{r}\right) & -\sin \left(\theta_{r}\right) & 0 \\
\sin \left(\theta_{r}\right) & \cos \left(\theta_{r}\right) & 0 \\
0 & 0 & 1
\end{array}\right)\left(\begin{array}{ccc}
1 & 0 & 0 \\
0 & \cos (\varphi) & -\sin (\varphi) \\
0 & \sin (\varphi) & \cos (\varphi)
\end{array}\right) \\
& =\left(\begin{array}{ccc}
\cos \left(\theta_{r}\right) & -\cos (\varphi) \sin \left(\theta_{r}\right) & \sin \left(\theta_{r}\right) \sin (\varphi) \\
\sin \left(\theta_{r}\right) & \cos (\varphi) \cos \left(\theta_{r}\right) & -\cos \left(\theta_{r}\right) \sin (\varphi) \\
0 & \sin (\varphi) & \cos (\varphi)
\end{array}\right)
\end{aligned}
$$

This differs slightly from [27] where $\varphi=0$ from the beginning. We believe this provides a convenient regularisation of the character, adapted to the flat limit. We can now compute the character:

$$
\begin{aligned}
\chi_{[m ; \vec{s}]}^{\text {Poinc. }([f, \alpha])} & =\int_{\mathcal{O}_{p}} d^{d} k \delta^{(d)}([\mathbb{1}-\Lambda] k) e^{i\langle k, \alpha\rangle} \chi_{\vec{s}}^{\mathfrak{s o}(d)}\left(g_{k}^{-1} \Lambda g_{k}\right) \\
& =e^{-\beta m} \frac{1}{\operatorname{det}|\mathbb{1}-\Lambda|} \chi_{\vec{s}}^{\mathfrak{s o}(d)}(\Lambda) \\
& =e^{-\beta m} \chi_{\vec{s}}^{\mathfrak{s o}(d)}(\vec{\theta}) \prod_{j=1}^{r} \frac{1}{\left|1-e^{i \theta_{j}}\right|^{2}}\left\{\begin{array}{cl}
1 & , \text { if } d=2 r \\
\left.\frac{1}{1-\cos \varphi}\right|_{\varphi \rightarrow 0}, & \text { if } d=2 r+1
\end{array}\right.
\end{aligned}
$$

where $\beta:=i \alpha_{0}$ and, to derive the last equality when $d=2 r+1$, we used:

$$
\begin{aligned}
\operatorname{det}\left|\mathbb{1}-R^{\prime}(\theta, \varphi)\right|= & (1-\cos \varphi)\left((1-\cos \theta)(1-\cos \varphi \cos \theta)+\cos \varphi \sin ^{2} \theta\right) \\
& -\sin \varphi\left((1-\cos \theta) \cos \theta \sin \varphi-\sin \varphi \sin ^{2} \theta\right) \\
\left.\Rightarrow \frac{1}{\operatorname{det}\left|\mathbb{1}-R^{\prime}(\theta, \varphi)\right|}\right|_{\varphi \rightarrow 0}= & \left.\frac{1}{2(1-\cos \theta)} \frac{1}{1-\cos \varphi}\right|_{\varphi \rightarrow 0}=\left.\frac{1}{\left|1-e^{i \theta}\right|^{2}} \frac{1}{1-\cos \varphi}\right|_{\varphi \rightarrow 0}
\end{aligned}
$$

Remark. When $\varphi=0, \Lambda$ is an element of the Cartan subgroup of $\operatorname{SO}(d)$. At the algebra level, the character is defined as:

$$
\chi_{V}(\mu)=\sum_{\tau \in \Delta_{V}} e^{\langle\tau, \mu\rangle}
$$


where $\Delta_{V}$ is the set of weights of the representation $V,\langle\cdot, \cdot\rangle$ is the scalar product on weight space and $\mu$ is an arbitrary weight. For semisimple algebras, the weight space has the structure of an Euclidean space, therefore one can write $e^{\langle\tau, \mu\rangle}=\prod_{j=1}^{r} x_{j}^{\tau_{j}}$ where $r$ is the dimension of the weight space (i.e. the rank of the algebra), $\tau_{j}$ the $j$ th component of the weight $\tau$ and $x_{j}:=e^{\mu_{j}}$. Hence, by definition, the above character encodes all the weights (i.e. eigenvalues of the Cartan subalgebra generators when acting on vectors in $V$ ) occurring in $V$. To compare the group character, one has to evaluate the latter on an element of the Cartan subgroup. The Cartan subalgebra being abelian, elements of the Cartan subgroup are of the form $\prod_{i=1}^{r} \exp \left(\theta_{i} H_{i}\right)$, where $H_{i}$ are the Cartan generators. Seeing the character as (a generalization of) the trace of a group element, it is clear that the character of an element of the Cartan subgroup will coincide with the Lie algebra character (E.14), upon identifying the parameter $\theta_{i}$ with the components $\mu_{i}$ of the weight $\mu$ on which the latter character is evaluated.

\section{E.2 Massless representations}

The massless case is a bit more subtle: in this case, the little group is the Euclidean group $\operatorname{ISO}(d-1)$. However, for "discrete" spin (or "helicity") representations, the translation are represented trivially and therefore the corresponding representation of $\operatorname{ISO}(d-1)$ reduces to a representation of $\mathrm{SO}(d-1)$. The characters corresponding to these massless, totally symmetric, spin-s representations were also computed in [27], however, when deriving them, one encounters a few difficulties in the form of divergences to be regularised. Even tough, as could be expected, the resulting formulae essentially contain the information about the irrep of the little group labeling these Poincaré UIRs in the form of a character of $\mathfrak{s o}(d-1)$, some regularising factors complicate the expression obtained and make their interpretation somewhat elusive. As the authors of [27] pointed out, the characters derived by the purely group theoretical approach do not exactly coincide with the corresponding flat spacetime partition functions computed using heat kernel method, despite the well known fact that the two objects are identical. It turns out that the character part of the partition functions spelled out in [27] are not plagued with as severe regularising factors as the corresponding ones obtained with the Frobenius formula outlined previously, and on top of that, arise naturally as flat limit of $\mathrm{AdS}_{d+1}$ characters. Having these facts in mind, we will assume that for massless Poincaré irreps, the characters are given by the result coming from partition function calculations, which reads:

$$
\chi_{[0 ; \vec{s}]}^{\text {Poinc. }}(\beta, \vec{\theta})=\prod_{j=1}^{r} \frac{1}{\mid 1-e^{\left.i \theta_{j}\right|^{2}}}\left\{\begin{array}{cl}
\sum_{k=1}^{r} \mathcal{A}_{k}^{(r)}(\vec{\theta}) \chi_{\vec{s}}^{\mathfrak{s o}(d-1)}\left(\hat{\theta}_{k}\right), & \text { if } d=2 r \\
\left.\frac{\chi_{\vec{s}}^{\mathfrak{s o}(d-1)}(\vec{\theta})}{1-\cos (\varphi)}\right|_{\varphi \rightarrow 0}, & \text { if } d=2 r+1 .
\end{array}\right.
$$

where $\hat{\theta}_{k}$ in the first line indicates that $\theta_{k}$ is removed. Making the identification $q=e^{-\beta}$ and $x_{j}=e^{i \theta_{j}}$, we recognise in the above formula the function $\mathscr{P}^{(d)}(\vec{x})$ defined in (3.13) and appearing as the flat limit of $\mathcal{P}^{(d)}(q, \vec{x})$ (the factor $\left.\frac{1}{1-\cos (\varphi)}\right|_{\varphi \rightarrow 0}$ should only be understood as a way of treating the divergence appearing in the expression of the character for 
$d=2 r+1$, and as such can be traded for $\left.\frac{1}{1-q}\right|_{q \rightarrow 1}$ appearing in the flat limit of (A)dS $\mathrm{dS}_{d+1}$ character, as both encode the same type of divergence to be regulated). We can therefore rewrite the Poincaré characters for massless irreps as:

$$
\chi_{[0 ; \vec{s}]}^{\text {Poinc. }}(q, \vec{x})=\mathscr{P}(d)(\vec{x})\left\{\begin{array}{cl}
\sum_{k=1}^{r} \mathcal{A}_{k}^{(r)}(\vec{x}) \chi_{\vec{s}}^{\mathfrak{s o}(d-1)}\left(\hat{\vec{x}}_{k}\right), & \text { if } d=2 r \\
\chi_{\vec{s}}^{\mathfrak{s o}(d-1)}(\vec{x}) \quad, \text { if } d=2 r+1,
\end{array}\right.
$$

where $\mathscr{P}^{(d)}(\vec{x})$ is defined by $(3.13)$ and $\hat{\vec{x}}_{k}:=\left(x_{1}, \ldots, x_{k-1}, x_{k+1}, \ldots, x_{r}\right)$.

\section{F Branching rules for $\mathfrak{s o}(d)$}

In this appendix, we derive the branching rules obeyed by $\mathfrak{s o}(d)$ characters. Before doing so, let us recall the expression of $\mathfrak{s o}(d)$ characters, written in terms of the $\xi$ and $\zeta$ variables, as well as the Vandermonde determinant $\Delta^{(r)}(\vec{\xi})$, used for instance in [72]:

$$
\zeta_{i}(\alpha):=x_{i}^{\alpha}-x_{i}^{-\alpha}, \quad \xi_{i}(\alpha):=x_{i}^{\alpha}+x_{i}^{-\alpha}, \quad \Delta^{(r)}(\vec{\xi})=\prod_{1 \leqslant i<j \leqslant r}\left(\xi_{i}-\xi_{j}\right) .
$$

Then, in terms of these building blocks, the characters of a $\mathfrak{s o}(d)$ irrep labelled by the highest-weight $\vec{s}=\left(s_{1}, \ldots, s_{r}\right)$ are:

- $d=2 r+1$ :

$$
\begin{aligned}
\chi_{\vec{s}}^{\mathfrak{s o}(2 r+1)}\left(x_{1}, \ldots, x_{r}\right) & =\frac{\prod_{k=1}^{r} \zeta_{k}^{-1}\left(\frac{1}{2}\right)}{\Delta^{(r)}(\vec{\xi})}\left|\begin{array}{cccc}
\zeta_{1}\left(s_{1}+r-\frac{1}{2}\right) & \zeta_{1}\left(s_{2}+r-\frac{3}{2}\right) & \ldots & \zeta_{1}\left(s_{r}+\frac{1}{2}\right) \\
\vdots & \vdots & \ddots & \vdots \\
\zeta_{r}\left(s_{1}+r-\frac{1}{2}\right) & \zeta_{r}\left(s_{2}+r-\frac{3}{2}\right) & \ldots & \zeta_{r}\left(s_{r}+\frac{1}{2}\right)
\end{array}\right| \\
& =\frac{1}{\Delta^{(r)}(\vec{\xi}) \prod_{k=1}^{r} \zeta_{k}\left(\frac{1}{2}\right)} \operatorname{det}\left(\zeta_{j}\left(s_{i}+r-i+\frac{1}{2}\right)\right)
\end{aligned}
$$

- $d=2 r$ :

$$
\begin{aligned}
& \chi_{\vec{s}}^{\mathfrak{s o}(2 r)}\left(x_{1}, \ldots, x_{r}\right)=\frac{1}{2 \Delta^{(r)}(\vec{\xi})}\left\{\left(\begin{array}{cccc}
\xi_{1}\left(s_{1}+r-1\right) & \ldots & \xi_{1}\left(s_{r-1}+1\right) & \xi_{1}\left(s_{r}\right) \\
\vdots & \ddots & \vdots & \vdots \\
\xi_{r}\left(s_{1}+r-1\right) & \ldots & \xi_{r}\left(s_{r-1}+1\right) & \xi_{r}\left(s_{r}\right)
\end{array} \mid\right.\right. \\
& \left.+\left|\begin{array}{cccc}
\zeta_{1}\left(s_{1}+r-1\right) & \ldots & \zeta_{1}\left(s_{r-1}+1\right) & \zeta_{1}\left(s_{r}\right) \\
\vdots & \ddots & \vdots & \vdots \\
\zeta_{r}\left(s_{1}+r-1\right) & \ldots & \zeta_{r}\left(s_{r-1}+1\right) & \zeta_{r}\left(s_{r}\right)
\end{array}\right|\right\} \\
& =\frac{1}{2 \Delta^{(r)}(\vec{\xi})}\left(\operatorname{det}\left(\xi_{j}\left(s_{i}+r-i\right)\right)+\operatorname{det}\left(\zeta_{j}\left(s_{i}+r-i\right)\right)\right)
\end{aligned}
$$

The branching rules for $\mathfrak{s o}(d)$, that we want to rederive at the character level, are at the level of irreps:

$$
\begin{aligned}
\mathcal{D}_{\vec{s}}^{\mathfrak{s o}(2 r+1)} & =\underset{s_{1} \geqslant \lambda_{1} \geqslant s_{2} \geqslant \cdots \geqslant \lambda_{r-1} \geqslant s_{r} \geqslant\left|\lambda_{r}\right|}{\bigoplus_{\vec{\lambda}}^{\mathfrak{s o}(2 r)}} \\
\mathcal{D}_{\vec{s}}^{\mathfrak{s o}(2 r)} & =\bigoplus_{s_{1} \geqslant \lambda_{1} \geqslant s_{2} \geqslant \cdots \geqslant \lambda_{r-1} \geqslant\left|s_{r}\right|} \mathcal{D}_{\vec{\lambda}}^{\mathfrak{s o}(2 r-1)}
\end{aligned}
$$


In the second formula, we denoted, with a slight abuse of notation, the $\mathfrak{s o}(2 r-1)$ weight by $\vec{\lambda}$ altough it actually stands for $\left(\lambda_{1}, \ldots, \lambda_{r-1}\right)$, i.e. a vector with $r-1$ components (since this is the rank of $\mathfrak{s o}(2 r-1))$. We will consider the two cases separately, starting with the odd dimensional one, and the main things we will need are the two identities gathered hereafter in a lemma.

\section{Lemma F.1.}

$$
\begin{aligned}
& \sum_{\mu=\lambda}^{\lambda^{\prime}} \zeta(\mu+\alpha)=\zeta^{-1}\left(\frac{1}{2}\right)\left(\xi\left(\lambda^{\prime}+\alpha+\frac{1}{2}\right)-\xi\left(\lambda+\alpha-\frac{1}{2}\right)\right) \\
& \sum_{\mu=\lambda}^{\lambda^{\prime}} \xi(\mu+\alpha)=\zeta^{-1}\left(\frac{1}{2}\right)\left(\zeta\left(\lambda^{\prime}+\alpha+\frac{1}{2}\right)-\zeta\left(\lambda+\alpha-\frac{1}{2}\right)\right)
\end{aligned}
$$

\section{F.1 $\mathfrak{s o}(2 r+1) \downarrow \mathfrak{s o}(2 r)$}

In odd dimensions, the branching rules at the character level reads:

$$
\chi_{\vec{s}}^{\mathfrak{s o}(2 r+1)}(\vec{x})=\sum_{\lambda_{1}=s_{2}}^{s_{1}} \cdots \sum_{\lambda_{r-1}=s_{r}}^{s_{r-1}} \sum_{\lambda_{r}=-s_{r}}^{s_{r}} \chi_{\vec{\lambda}}^{\mathfrak{s o}(2 r)}(\vec{x})
$$

Proof. Having in mind the character of the $\mathfrak{s o}(2 r+1)$ irrep $\vec{s}$ :

$$
\chi_{\vec{s}}^{\mathfrak{s o}(2 r+1)}(\vec{x})=\frac{1}{\Delta^{(r)}(\vec{\xi})} \sum_{\sigma \in \mathcal{S}_{r}} \varepsilon(\sigma) \prod_{i=1}^{r} \zeta_{\sigma(i)}^{-1}\left(\frac{1}{2}\right) \zeta_{\sigma(i)}\left(s_{i}+r-i+\frac{1}{2}\right),
$$

with $\varepsilon(\sigma)$ the signature of the permutation $\sigma$, let us rewrite the sum of $\mathfrak{s o}(2 r)$ of the irreps appearing in the branching rule of $\vec{s}$ :

$$
\begin{aligned}
\sum_{\lambda_{1}=s_{2}}^{s_{1}} & \ldots \sum_{\lambda_{r-1}=s_{r}}^{s_{r-1}} \sum_{\lambda_{r}=-s_{r}}^{s_{r}} \chi_{\vec{\lambda}}^{\mathfrak{s o}(2 r)}(\vec{x}) \\
= & \frac{1}{2 \Delta^{(r)}(\vec{\xi})} \sum_{\sigma \in \mathcal{S}_{r}} \varepsilon(\sigma) \prod_{i=1}^{r}\left(\sum_{\lambda_{r}=-s_{r}}^{s_{r}} \cdots \sum_{\lambda_{1}=s_{2}}^{s_{1}} \xi_{\sigma(i)}\left(\lambda_{i}+r-i\right)\right) \\
= & \frac{\prod_{k=1}^{r} \zeta_{k}^{-1}\left(\frac{1}{2}\right)}{2 \Delta^{(r)}(\vec{\xi})} \sum_{\sigma \in \mathcal{S}_{r}} \varepsilon(\sigma) \prod_{i=1}^{r-1}\left(\zeta_{\sigma(i)}\left(s_{i}+r-i+\frac{1}{2}\right)-\zeta_{\sigma(i)}\left(s_{i+1}+r-i-\frac{1}{2}\right)\right) \\
& \quad \times\left(\zeta_{\sigma(r)}\left(s_{r}+\frac{1}{2}\right)-\zeta_{\sigma(r)}\left(-s_{r}-\frac{1}{2}\right)\right)
\end{aligned}
$$

where we used Lemma F.1 to obtain the final line. Notice that $\zeta(-x)=-\zeta(x)$, as follows from the definition, therefore only the determinant involving the variables $\xi$ in (F.5) survives in the expression of the $\mathfrak{s o}(2 r)$ characters in the sum to begin with, and the last factor above becomes $2 \zeta_{\sigma(r)}\left(s_{r}+\frac{1}{2}\right)$. Finally, only the term $\prod_{i=1}^{r} \zeta_{\sigma(r)}\left(s_{i}+r-i+\frac{1}{2}\right)$ in the product of the previous expression remains. Indeed, the second terms of the difference inside this product, namely $\zeta_{\sigma(i)}\left(s_{i+1}+r-i-\frac{1}{2}\right)$ gives rise to terms of the form 
$\zeta_{\sigma(i)}\left(s_{i+1}+r-i-\frac{1}{2}\right) \zeta_{\sigma(i+1)}\left(s_{i+1}+r-i-\frac{1}{2}\right)$ when expanding the product. These terms will automatically be cancelled when summing over all permutations, as there will always be two permutations $\sigma$ and $\sigma^{\prime}$ such that for a fixed $i \in\{1,2, \cdots, r\}, \sigma(i)=\sigma^{\prime}(i+1), \sigma(i+1)=\sigma^{\prime}(i)$ and $\sigma(j)=\sigma^{\prime}(j), \forall j \neq i$, and by definition the signature of $\sigma$ and $\sigma^{\prime}$ differ by a minus sign. As a consequence, the above equation yields:

$$
\begin{aligned}
\sum_{\lambda_{1}=s_{2}}^{s_{1}} \cdots \sum_{\lambda_{r}=-s_{r}}^{s_{r}} \chi_{\vec{\lambda}}^{\mathfrak{s o}(2 r)}(\vec{x}) & =\frac{1}{\Delta^{(r)}(\vec{\xi}) \prod_{k=1}^{r} \zeta_{k}\left(\frac{1}{2}\right)} \sum_{\sigma \in \mathcal{S}_{r}} \varepsilon(\sigma) \prod_{i=1}^{r} \zeta_{\sigma(i)}\left(s_{i}+r-i+\frac{1}{2}\right) \\
& =\chi_{\vec{s}}^{\mathfrak{s o}(2 r+1)}(\vec{x}),
\end{aligned}
$$

which proves (F.10).

Example 5. Consider the simple, low rank, case of $\mathfrak{s o}(5) \downarrow \mathfrak{s o}(4)$ :

- On the one hand,

$$
\chi_{(s, t)}^{\mathfrak{s o}(5)}\left(x_{1}, x_{2}\right)=\frac{1}{\Delta^{(2)}(\vec{\xi}) \zeta_{1}\left(\frac{1}{2}\right) \zeta_{2}\left(\frac{1}{2}\right)}\left(\zeta_{1}\left(s+\frac{3}{2}\right) \zeta_{2}\left(t+\frac{1}{2}\right)-\zeta_{1}\left(t+\frac{1}{2}\right) \zeta_{2}\left(s+\frac{3}{2}\right)\right)
$$

- On the other hand,

$$
\begin{gathered}
\sum_{\sigma=t}^{s} \sum_{\tau=-t}^{t} \chi_{(\sigma, \tau)}^{\mathfrak{s o}(4)}\left(x_{1}, x_{2}\right)=\frac{1}{2 \Delta^{(2)}(\vec{\xi})} \sum_{\sigma=t}^{s} \sum_{\tau=-t}^{t}\left(\xi_{1}(\sigma+1) \xi_{2}(\tau)-\xi_{2}(\sigma+1) \xi_{1}(\tau)\right) \\
=\frac{1}{2 \Delta^{(2)}(\vec{\xi}) \zeta_{1}\left(\frac{1}{2}\right) \zeta_{2}\left(\frac{1}{2}\right)}\left[\left(\zeta_{1}\left(s+\frac{3}{2}\right)-\zeta_{1}\left(t+\frac{1}{2}\right)\right)\left(\zeta_{2}\left(t+\frac{1}{2}\right)-\zeta_{2}\left(-t-\frac{1}{2}\right)\right)\right. \\
\left.\quad-\left(\zeta_{1}\left(t+\frac{1}{2}\right)-\zeta_{1}\left(-t-\frac{1}{2}\right)\right)\left(\zeta_{2}\left(s+\frac{3}{2}\right)-\zeta_{2}\left(t+\frac{1}{2}\right)\right)\right]
\end{gathered}
$$

The terms $\zeta_{1}\left(t+\frac{1}{2}\right) \zeta_{2}\left(t+\frac{1}{2}\right)$ cancel, and using $\zeta(-x)=-\zeta(x)$, we are left with:

$$
\chi_{(s, t)}^{\mathfrak{s o}(5)}\left(x_{1}, x_{2}\right)=\sum_{\sigma=t}^{s} \sum_{\tau=-t}^{t} \chi_{(\sigma, \tau)}^{\mathfrak{s o}(4)}\left(x_{1}, x_{2}\right)
$$

\section{F.2 $\mathfrak{s o}(2 r) \downarrow \mathfrak{s o}(2 r-1)$}

In even dimensions, the branching rule at the character level reads:

$$
\chi_{\vec{s}_{+}}^{\mathfrak{s o}(2 r)}(\vec{x})+\chi_{\vec{s}_{-}}^{\mathfrak{s o}(2 r)}(\vec{x})=\sum_{k=1}^{r} \mathcal{A}_{k, \vec{s}}^{(r)}(\vec{x}) \sum_{\lambda_{1}=s_{2}}^{s_{1}} \ldots \sum_{\lambda_{r-1}=s_{r}}^{s_{r-1}} \chi_{\vec{\lambda}}^{\mathfrak{s o}(2 r-1)}\left(\hat{\vec{x}}_{k}\right),
$$

with $\vec{s}_{ \pm}=\left(s_{1}, \ldots, \pm s_{r}\right), \hat{\vec{x}}_{k}:=\left(x_{1}, \ldots, x_{k-1}, x_{k+1}, \ldots, x_{r}\right)$ and

$$
\mathcal{A}_{k, \vec{s}}^{(r)}\left(x_{1}, \ldots, x_{r}\right):=\xi_{k}\left(s_{r}\right) \frac{\left.\Delta^{(r)}(\vec{\xi})\right|_{\xi_{k}=2}}{\Delta^{(r)}(\vec{\xi})}
$$

Notice that when $s_{r}=0, \xi_{k}\left(s_{r}\right)=2$ and the above identity reduces to a statement involving only one character. 
Proof. We can rewrite the sum of the characters for $\vec{s}_{+}$and $\vec{s}_{-}$, by explicitly expanding the only remaining determinant, the one involving the variables $\xi$. Indeed, either the last component $s_{r}$ of $s_{ \pm}$vanishes, hence a whole column in $\operatorname{det}\left(\zeta_{j}\left(s_{r}+r-i\right)\right)$ vanishes and thereby the whole determinant vanishes; or the two determinants involving the variables $\zeta$ will cancel each other as the last column of one will be $\zeta_{i}\left(s_{r}\right)$ and $\zeta_{i}\left(-s_{r}\right)=-\zeta_{i}\left(s_{r}\right)$ for the other one. The resulting sum of characters reads:

$$
\begin{gathered}
\chi_{\vec{s}_{+}}^{\mathfrak{s o}(2 r)}(\vec{x})+\chi_{\vec{s}_{-}}^{\mathfrak{s o}(2 r)}(\vec{x})=\frac{1}{\Delta^{(r)}(\vec{\xi})}\left|\begin{array}{cccc}
\xi_{1}\left(s_{1}+r-1\right) & \ldots & \xi_{1}\left(s_{r-1}+1\right) & \xi_{1}\left(s_{r}\right) \\
\vdots & \ddots & \vdots & \vdots \\
\xi_{r}\left(s_{1}+r-1\right) & \ldots & \xi_{r}\left(s_{r-1}+1\right) & \xi_{r}\left(s_{r}\right)
\end{array}\right| \\
=\frac{1}{\Delta^{(r)}(\vec{\xi})} \sum_{k=1}^{r}(-)^{k+r} \xi_{k}\left(s_{r}\right)\left(\begin{array}{ccc}
\sum_{\sigma \in \mathcal{S}_{r-1}} \varepsilon(\sigma) & \prod_{i \in\{1,2, \cdots, r\}, i \neq k} \xi_{\sigma(i)}\left(s_{i}+r-i\right)
\end{array}\right),
\end{gathered}
$$

One the other hand, the sum of the $\mathfrak{s o}(2 r-1)$ characters corresponding to the irreps appearing in the branching rule of $\vec{s}_{ \pm}$reads:

$$
\begin{aligned}
& \sum_{\lambda_{r-1}=0}^{s_{r-1}} \sum_{\lambda_{r-2}=s_{r-1}}^{s_{r-2}} \cdots \sum_{\lambda_{1}=s_{2}}^{s_{1}} \chi_{\vec{\lambda}}^{\mathfrak{s o}(2 r-1)}(\vec{x}) \\
= & \frac{1}{\Delta^{(r-1)}(\vec{\xi})} \sum_{\sigma \in \mathcal{S}_{r-1}} \varepsilon(\sigma) \prod_{i=1}^{r-1} \zeta_{\sigma(i)}^{-1}\left(\frac{1}{2}\right) \sum_{\lambda_{r-1}=0}^{s_{r-1}} \sum_{\lambda_{r-2}=s_{r-1}}^{s_{r-2}} \ldots \sum_{\lambda_{1}=s_{2}}^{s_{1}} \zeta_{\sigma(i)}\left(\lambda_{i}+r-\frac{1}{2}\right) \\
= & \frac{1}{\Delta^{(r-1)}(\vec{\xi})} \sum_{\sigma \in \mathcal{S}_{r-1}} \varepsilon(\sigma) \prod_{i=1}^{r-1} \zeta_{\sigma(i)}^{-2}\left(\frac{1}{2}\right)\left(\xi_{\sigma(i)}\left(s_{i}+r-i\right)-\xi_{\sigma(i)}\left(s_{i+1}+r-[i+1]\right)\right)
\end{aligned}
$$

At this point, one can notice the following identities:

$$
\zeta^{2}\left(\frac{1}{2}\right)=\left(x^{1 / 2}-x^{-1 / 2}\right)^{2}=x+x^{-1}-2=\xi(1)-2,
$$

and

$$
\begin{aligned}
\left.\Delta^{(r)}(\vec{\xi})\right|_{\xi_{k}=2} & =\prod_{1 \leqslant i<j \leqslant r, i, j \neq k}\left(\xi_{i}-\xi_{j}\right) \prod_{n=1}^{k-1}\left(\xi_{n}-2\right) \prod_{m=k+1}^{r}\left(2-\xi_{m}\right) \\
& =(-)^{r+k} \prod_{1 \leqslant i \leqslant r, i \neq k}\left(\xi_{i}-2\right) \Delta^{(r-1)}\left(\hat{\overrightarrow{\xi_{k}}}\right)=(-)^{k+r} \prod_{1 \leqslant i \leqslant r, i \neq k} \zeta_{i}^{2}\left(\frac{1}{2}\right) \Delta^{(r-1)}\left(\hat{\vec{\xi}}_{k}\right)
\end{aligned}
$$

The above sum of $\mathfrak{s o}(2 r-1)$ can therefore be rewritten as:

$$
\begin{aligned}
& \sum_{\lambda_{r-1}=0}^{s_{r-1}} \sum_{\lambda_{r-2}=s_{r-1}}^{s_{r-2}} \cdots \sum_{\lambda_{1}=s_{2}}^{s_{1}} \chi_{\vec{\lambda}}^{\mathfrak{s o}(2 r-1)}(\vec{x})= \\
& \quad=\frac{1}{\left.\Delta^{(r)}(\vec{\xi})\right|_{\xi_{r}=2}} \sum_{\sigma \in \mathcal{S}_{r-1}} \varepsilon(\sigma) \prod_{i=1}^{r-1}\left(\xi_{\sigma(i)}\left(s_{i}+r-i\right)-\xi_{\sigma(i)}\left(s_{i+1}+r-[i+1]\right)\right)
\end{aligned}
$$


Now taking the following linear combination, and using (F.26):

$$
\begin{aligned}
\sum_{k=1}^{r} \frac{\left.\Delta^{(r)}(\vec{\xi})\right|_{\xi_{k}=2}}{\Delta^{(r)}(\vec{\xi})} \xi_{k}\left(s_{r}\right) \sum_{\lambda_{r-1}=0}^{s_{r-1}} \cdots \sum_{\lambda_{1}=s_{2}}^{s_{1}} \chi_{\vec{\lambda}}^{\mathfrak{s o}(2 r-1)}\left(\hat{\vec{x}}_{k}\right)= \\
=\frac{1}{\Delta^{(r)}(\vec{\xi})} \sum_{k=1}^{r}(-)^{k+r} \xi_{k}\left(s_{r}\right) \sum_{\sigma \in \mathcal{S}_{r-1}} \varepsilon(\sigma) \\
\quad \times \prod_{i \in\{1,2, \cdots, r\}, i \neq k}\left(\xi_{\sigma(i)}\left(s_{i}+r-i\right)-\xi_{\sigma(i)}\left(s_{i+1}+r-[i+1]\right)\right),
\end{aligned}
$$

one can easily recognise the sum $\chi_{\overrightarrow{s_{+}}}^{\mathfrak{s o}(2 r)}(\vec{x})+\chi_{\vec{s}_{-}}^{\mathfrak{s o}(2 r)}(\vec{x})$ by isolating the contribution $\prod_{i \in\{1, \cdots, r\}, i \neq k} \xi_{\sigma(i)}\left(s_{i}+r-i\right)$ in the expansion of the final product. It turns out that all the other terms in this expansion cancel one another for the same reason as in the previous case $\mathfrak{s o}(2 r+1) \downarrow \mathfrak{s o}(2 r)$ : the remaining terms are of the form $\xi_{\sigma(i)}\left(k_{j}\right) \xi_{\sigma(l)}\left(k_{j}\right)$ and one can check that there will always be two permutations $\sigma$ and $\sigma^{\prime}$ only exchanging $i$ and $l$ and whose signature differs by a minus sign.

Example 6. Consider the simple, low rank, case of $\mathfrak{s o}(4) \downarrow \mathfrak{s o}(3)$ :

- On the one hand:

$$
\begin{gathered}
\chi_{(s, t)}^{\mathfrak{s o}(4)}\left(x_{1}, x_{2}\right)+\chi_{(s,-t)}^{\mathfrak{s o}(4)}\left(x_{1}, x_{2}\right)=\frac{1}{\Delta^{(2)}(\vec{\xi})}\left(\xi_{1}(s+1) \xi_{2}(t)-\xi_{1}(t) \xi_{2}(s+1)\right) \\
=\frac{1}{\Delta^{(2)}(\vec{\xi})}\left(\left[\xi_{1}(s+1)-\xi_{1}(t)\right] \xi_{2}(t)-\xi_{1}(t)\left[\xi_{2}(s+1)-\xi_{2}(t)\right]\right)
\end{gathered}
$$

- On the other hand:

$$
\chi_{(\sigma)}^{\mathfrak{s o}(3)}(x)=\frac{\zeta\left(\sigma+\frac{1}{2}\right)}{\zeta\left(\frac{1}{2}\right)} \Rightarrow \sum_{\sigma=t}^{s} \chi_{(\sigma)}^{\mathfrak{s o}(3)}(x)=\zeta^{-2}\left(\frac{1}{2}\right)(\xi(s+1)-\xi(t)) .
$$

Putting this altogether, we end up with:

$$
\chi_{(s, t)}^{\mathfrak{s o}(4)}\left(x_{1}, x_{2}\right)+\chi_{(s,-t)}^{\mathfrak{s o}(4)}\left(x_{1}, x_{2}\right)=\sum_{\sigma=t}^{s} \frac{\Delta^{(2)}(\vec{\xi}) \xi_{1}=2}{\Delta^{(2)}(\vec{\xi})} \xi_{2}(t) \chi_{(\sigma)}^{\mathfrak{s o}(3)}\left(x_{2}\right)+\frac{\Delta^{(2)}(\vec{\xi})_{\xi_{2}=2}}{\Delta^{(2)}(\vec{\xi})} \xi_{1}(t) \chi_{(\sigma)}^{\mathfrak{s o}(3)}\left(x_{1}\right)
$$

\section{G Non-unitary mixed-symmetry massless fields}

In this appendix we spell out the characters corresponding to the non-unitary massless mixed-symmetry fields in both de Sitter and anti-de Sitter, and comment on their flat limit as well. 


\section{G.1 Anti-de Sitter case}

As shown by Metsaev [8, 9], massless mixed-symmetry fields in anti-de Sitter are unitary only when their first block is "activated" by gauge transformations, i.e. its gauge parameter takes values in the $\mathfrak{s o}(d)$ Young diagram obtained by removing one box in the last row of the first block of the Young diagram of the gauge field. However, if one ignores the unitarity of the representation and is only interested in its irreducibility, all intermediary block of the gauge field's Young diagram can be activated.

The conformal weight of a gauge field with symmetry $\mathbb{Y}=\left(\ell_{1}^{h_{1}}, \ldots, \ell_{B}^{h_{B}}\right)$ (a diagram with $B$ blocks of respective lengths $\ell_{I}$ ) whose $I$ th block is activated is $\Delta_{I}:=\ell_{I}+d-p_{I}-1$, where $p_{I}:=\sum_{J=1}^{I} h_{J}$ is the cumulated height of the $I$ first blocks. This $\mathfrak{s o}(2) \oplus \mathfrak{s o}(d)$ highest-weight can be found in the BGG sequences for $\mathfrak{s o}(2, d)$ detailed in appendix D at level $(\lambda)_{d-p_{I}}$ for $d=2 r+1$ and at level $(\lambda)_{r-p_{I}}$ for $d=2 r$. Using the general formulae derived in appendix $\mathrm{D}$, we can therefore write down the corresponding character:

$$
\chi_{\left[\Delta_{I}, \mathbb{Y}\right]}^{\operatorname{AdS}}(q, \vec{x})=q^{\Delta_{I}} \chi_{\mathbb{Y}}^{\mathfrak{s o}(d)}(\vec{x}) \mathcal{P}^{(d)}(\vec{x})+\sum_{k=1}^{p_{I}}(-1)^{p_{I}+k+1} q^{\ell_{k}+d-k} \chi_{\breve{Y}_{I}^{(k)}}^{\mathfrak{s o}(d)}(\vec{x}) \mathcal{P}^{(d)}(\vec{x})
$$

where $\check{Y}_{I}^{(k)}$ is obtained from $\mathbb{Y}$ by (i) adding an additional row to the $I$ th block (of the same length, i.e. $\ell_{I}$ ) and (ii) deleting the $k$ th row in this new diagram together with removing one box in each of the rows under the one just removed and until the $p_{I}$ th (i.e. the end of the $I$ th block). More explicitly:

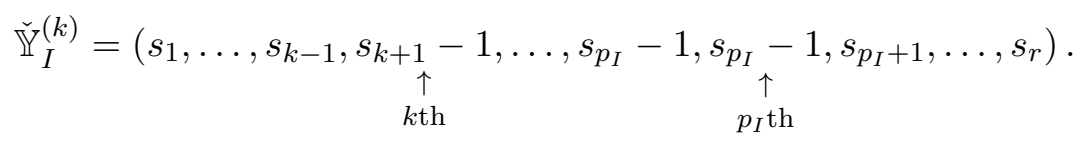

Again, when taking the flat limit of these characters one obtains first a sequence of $\mathfrak{s o}(d)$ irreps to branch onto $\mathfrak{s o}(d-1)$, corresponding to the Young diagrams of the massless fields, its gauge parameter and its reducibility. This precise combination of $\mathfrak{s o}(d)$-irreps in $(\mathrm{G} .1)$ is such that, when branched onto $\mathfrak{s o}(d-1)$, it produces all possible Young diagrams resulting from the branching rule of the gauge field Young diagram where the block activated by the gauge symmetry is left untouched. The proof is identical to the analysis performed in subsection 3.3 when deriving the flat limit of exceptional series UIRs, and therefore we will not reproduce it here. The spectrum of massless fields produced by taking the flat limit of a single non-unitary mixed-symmetry field with Young diagram $\mathbb{Y}=\left(\ell_{1}^{h_{1}}, \ldots, \ell_{B}^{h_{B}}\right)$ in $\operatorname{AdS}_{d+1}$ whose $I$ th block is touched by gauge symmetry is therefore:

$$
\begin{gathered}
\Sigma(\mathbb{Y})=\left\{\mathbb{Y}^{\prime}=\left(\ell_{1}^{h_{1}-1}, \ell_{1}-n_{1}, \ldots, \ell_{I-1}^{h_{I-1}}, \ell_{I-1}-n_{I-1}, \ell_{I}^{h_{I}}, \ell_{I+1}^{h_{I+1}}, \ell_{I+1}-n_{I+1}, \ldots, \ell_{B}^{h_{B}-1}, \ell_{B}-n_{B}\right),\right. \\
\left.0 \leqslant n_{i} \leqslant s_{i}-s_{i+1}, i \in\{1,2, \cdots, B\}, i \neq I\right\} .
\end{gathered}
$$

Example 7. Let us consider a gauge field with mixed-symmetry given by $\mathbb{Y}=\boxplus$ whose second block is activated, thus with conformal weight $\Delta_{I=2}=d-2$ since $\ell_{2}=2$ and $p_{2}=3$. 
Its character reads:

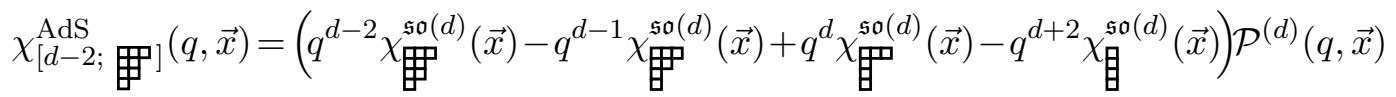

$$
\begin{aligned}
& \underset{\lambda \rightarrow 0}{\longrightarrow}\left(\chi_{\text {四 }}^{\mathfrak{s o}(d)}(\vec{x})-\chi_{\mathbb{\Xi}}^{\mathfrak{s o}(d)}(\vec{x})+\chi_{\text {目 }}^{\mathfrak{s o}(d)}(\vec{x})-\chi_{\text {目 }}^{\mathfrak{s o}(d)}(\vec{x})\right) \mathscr{P}^{(d)}(\vec{x})
\end{aligned}
$$

Branching all diagrams appearing in the previous formula (and sorting the result by lexicographic ordering):

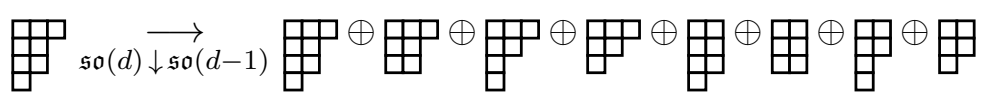

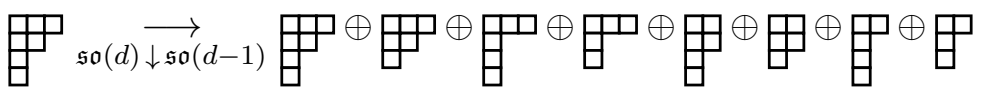

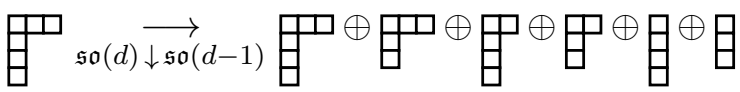

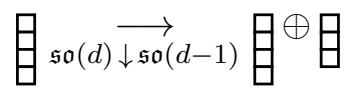

One is therefore left with:

$$
\begin{aligned}
& \chi_{[d-1 ; \text { 田 }}^{\operatorname{AdS}}(q, \vec{x}) \underset{\lambda \rightarrow 0}{\longrightarrow}\left(\chi_{\text {卢 }}^{\mathfrak{s o}(d)}(\vec{x})+\chi_{\text {田 }}^{\mathfrak{s o}(d)}(\vec{x})+\chi_{\text {困 }}^{\mathfrak{s o}(d)}(\vec{x})+\chi_{\text {田 }}^{\mathfrak{s o}(d)}(\vec{x})\right) \mathscr{P}^{(d)}(\vec{x}) \\
& =\chi_{[0 ; \text { 田 }}^{\text {Poinc. }}(\vec{x})+\chi_{[0 ; \text { 田 }]}^{\text {Poinc. }}(\vec{x})+\chi_{[0 ; \text { 田 }}^{\text {Poinc }}(\vec{x})+\chi_{[0 ; \text { 田 }]}^{\text {Poinc }}(\vec{x}),
\end{aligned}
$$

i.e. as expected, only appear massless fields with Young diagrams obtained from branching $\mathbb{Y}$ from $\mathfrak{s o}(d)$ onto $\mathfrak{s o}(d-1)$ with the exception of leaving the second block untouched.

\section{G.2 de Sitter case}

Irreducible representations of $\mathfrak{s o}(1, d+1)$ were (to our knowledge) first spelled out in $[19,73]$ then completed in $[20,31,38]$. In these early papers, one can find the classification of irreps, irrespectively of their unitary character.

Irreps of the exceptional series are labeled by $[19,73]$ the conformal weight $\Delta_{c}=$ $d+n-p_{I}-1$, and a Young diagram $\mathbb{Y}_{p_{I}}=\left(s_{1}, \ldots, s_{r}\right)$, such that $s_{p_{I}+1}>n \geqslant$ $s_{p_{I}+2}, n \in \mathbb{N}$. This set of data should describe a gauge field with symmetry $\mathbb{Y}_{n, p_{I}}:=$ $\left(s_{1}, \ldots, s_{p_{I}}, n, s_{p_{I}+2}, \ldots, s_{B}\right)$ whose $I$ th block is activated (having in mind that as in the previous subsection, $p_{I}$ is the cumulated height of the first $I$ blocks of this diagram, whose total height is $\left.p_{B}\right)$ whose gauge parameter has symmetry $\check{\mathbb{Y}}_{n, p_{I}}^{\left(p_{I}\right)}:=\left(s_{1}, \ldots, s_{p_{I}-1}, s_{p_{I}+1}-\right.$ $\left.1, s_{p_{I}+2}, \ldots, s_{p_{B}}\right)$. Those representations are unitary only for $n=0$, that is when the activated block is the last one. More generically, the characters of the exceptional series are: 
- Odd spacetime dimensions:

$$
\begin{aligned}
& \chi_{\left[d+n-p_{I}-1 ; \mathbb{Y}_{n, p_{I}}\right]}^{\mathrm{dS}}(q, \vec{x})=\left(q^{p_{I}+1-n}-q^{d-p_{I}-1+n}\right) \chi_{\mathbb{Y}_{p_{I}}^{\mathfrak{s o}(d)}}(\vec{x}) \mathcal{P}^{(d)}(q, \vec{x}) \\
& +\sum_{m=1}^{p_{B}-p_{I}}(-1)^{m}\left(q^{p_{I}+1+m-s_{p_{I}+1+m}}-q^{d-p_{I}-1-m+s_{p_{I}+1+m}}\right) \chi_{\hat{\mathbb{Y}}_{n, p_{I}}^{\mathfrak{s o}(m)}(\vec{x})}^{(\vec{x})} \mathcal{P}^{(d)}(q, \vec{x})
\end{aligned}
$$

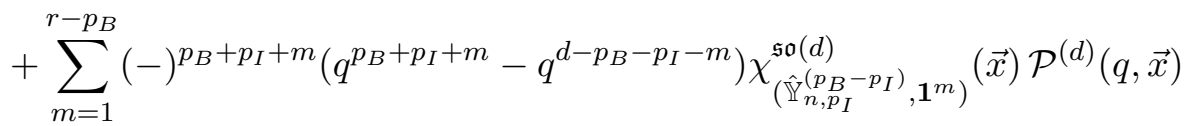

$$
\begin{aligned}
& +\sum_{\ell=1}^{p_{I}+1}(-)^{\ell+p_{I}+1} q^{s_{\ell}+d-\ell} \chi_{\check{Y}_{n, p_{I}}^{(\ell)}(d)}^{(\ell)}(\vec{x}) \mathcal{P}^{(d)}(q, \vec{x}),
\end{aligned}
$$

where $\hat{\mathbb{Y}}_{n, p_{I}}^{(m)}$ is the Young diagram obtained by adding one box in each of the $m$ row under the $\left(p_{I}+1\right)$ th of $\mathbb{Y}_{n, p_{I}}$, i.e.

$$
\hat{\mathbb{Y}}_{n, p_{I}}^{(m)}=\left(s_{1}, \ldots, s_{p_{I}}, n+1, s_{p_{I}+2}+1, \ldots, s_{p_{I}+m}+1, s_{p_{I}+m+1}, \ldots, s_{p_{B}}, 0, \ldots, 0\right)
$$

and $\check{\mathbb{Y}}_{n, p_{I}}^{(\ell)}$ is the diagram obtained by removing the $\ell$ th row together with one box in each of the rows after the $\ell$ th one until the $\left(p_{I}+1\right)$ th from $\mathbb{Y}_{n, p_{I}}$,

$$
\check{Y}_{n, p_{I}}^{(\ell)}=\left(s_{1}, \ldots, s_{\ell-1}, s_{\ell+1}-1, \ldots, s_{p_{I}+1}-1, n, s_{p_{I}+2}, \ldots, s_{p_{B}}, 0, \ldots, 0\right) .
$$

Taking the flat limit $(q \rightarrow 1)$ of the above expression, one is left with an alternating sum of $\mathfrak{s o}(d)$ characters of the same type as in the unitary case or the above detailed $\operatorname{AdS}_{d+1}$ case:

$$
\chi_{\left[d+n-p_{I}-1 ; \mathbb{Y}_{n, p_{I}}\right]}^{\mathrm{dS}}(q, \vec{x}) \underset{\lambda \rightarrow 0}{\longrightarrow} \sum_{\ell=1}^{p_{I}+1}(-)^{\ell+p_{I}+1} \chi_{\widetilde{\mathbb{Y}}_{n, p_{I}}^{(\ell)}}^{\mathfrak{s o}(d)}(\vec{x})
$$

The Young diagrams appearing in this sum correspond to a gauge field with symmetry $\mathbb{Y}_{n, p_{I}}$, its gauge parameter having symmetry $\check{\mathbb{Y}}_{n, p_{I}}^{\left(p_{I}\right)}$ and its reducibility parameters, and therefore by the same arguments used in subsection 3.3 one is left with the following spectrum of massless fields in flat space:

$$
\begin{aligned}
& \Sigma\left(\mathbb{Y}_{n, p_{I}}\right) \\
& =\left\{\mathbb{Y}^{\prime}=\left(\ell_{1}^{h_{1}-1}, \ell_{1}-n_{1}, \ldots, \ell_{I-1}^{h_{I-1}-1}, \ell_{I-1}-n_{I-1}, \ell_{I}^{h_{I}}, n-n_{p}, \ell_{I+1}^{h_{I+1}-1},\right.\right. \\
& \\
& \left.\quad \ell_{I+1}-n_{I+1}, \ldots, \ell_{B}^{h_{B}-1}, \ell_{B}-n_{B}\right), \\
& \left.\quad 0 \leqslant s_{i}-s_{i+1}, i \in\{1, \cdots, B\}, i \neq I, 0 \leqslant n_{p} \leqslant n-s_{I+1}\right\}
\end{aligned}
$$

Remark. From our earlier analysis of the unitary irreps of the exceptional series, we learned that the character obtained from resolving the module of the shadow of what we called the gauge field's curvature (using the BGG sequences recalled in appendix D) matches the character obtained in [36]. We therefore applied the same technique for non-unitary representation, i.e. we computed the character corresponding to generalized Verma module with highest-weight $\left[\Delta_{c} ; \vec{s}\right]=\left[p_{I}+1-n ; \mathbb{Y}_{p_{I}}\right]$ (remember that the curvature is characterized by the same Young diagram and conformal weight $d-\Delta_{c}$ ). 
- Even spacetime dimensions:

$$
\begin{aligned}
& \chi_{\left[d+n-p_{I}-1 ; \mathbb{Y}_{\left.n, p_{I}\right]}\right]}^{\mathrm{dS}}(q, \vec{x})=\left(q^{p_{I}+1-n}+q^{d-p_{I}-1+n}\right) \chi_{\mathbb{Y}_{n, p_{I}}}^{\mathfrak{s o}(d)}(\vec{x}) \mathcal{P}^{(d)}(q, \vec{x}) \\
& +\sum_{m=1}^{p_{B}-p_{I}}(-1)^{m}\left(q^{p_{I}+1+m-s_{p_{I}+1-m}}+q^{d-p_{I}-1-m+s_{p_{I}+1-m}}\right) \chi_{\hat{\mathbb{Y}}_{n, p_{I}}^{(m)}(d)}^{\mathfrak{s o}(\vec{x})} \mathcal{P}^{(d)}(q, \vec{x}) \\
& +\sum_{m=1}^{r-p_{B}-1}(-)^{p_{B}+p_{I}+m}\left(q^{p_{B}+m}+q^{d-p_{B}-m}\right) \chi_{\left(\hat{\mathbb{Y}}_{n, p_{I}}^{\left(p_{B}-p_{I}\right)}, \mathbf{1}^{m}\right)}^{\mathfrak{s}(d)}(\vec{x}) \mathcal{P}^{(d)}(q, \vec{x})
\end{aligned}
$$

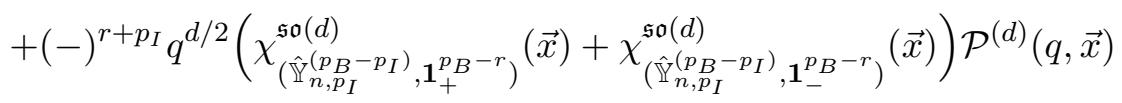

$$
\begin{aligned}
& -2 \sum_{\ell=1}^{p_{I}+1}(-)^{\ell+p_{I}+1} q^{s_{\ell}+d-\ell} \chi_{\mathbb{Y}_{n, p_{I}}^{(\ell)}(d)}^{\mathfrak{s o}}(\vec{x}) \mathcal{P}^{(d)}(q, \vec{x}),
\end{aligned}
$$

For the same reason as in the case of UIRs in the exceptional series in even spacetime dimensions treated in subsection 3.3, the flat limit of the character of their nonunitary counterpart does not appear to produce a result that can be interpreted as a sum of Poincaré characters for massless fields that could be part of a BMV-type mechanism.

Open Access. This article is distributed under the terms of the Creative Commons Attribution License (CC-BY 4.0), which permits any use, distribution and reproduction in any medium, provided the original author(s) and source are credited.

\section{References}

[1] J.M.F. Labastida, Massless particles in arbitrary representations of the Lorentz group, Nucl. Phys. B 322 (1989) 185 [inSPIRE].

[2] J.M.F. Labastida, Massless fermionic free fields, Phys. Lett. B 186 (1987) 365 [INSPIRE].

[3] W. Siegel and B. Zwiebach, Gauge string fields from the light cone, Nucl. Phys. B 282 (1987) 125 [INSPIRE].

[4] X. Bekaert and N. Boulanger, Tensor gauge fields in arbitrary representations of $\operatorname{GL}(D, R)$ : duality and Poincaré lemma, Commun. Math. Phys. 245 (2004) 27 [hep-th/0208058] [INSPIRE].

[5] X. Bekaert and N. Boulanger, Tensor gauge fields in arbitrary representations of $\operatorname{GL}(D, R)$ II. Quadratic actions, Commun. Math. Phys. 271 (2007) 723 [hep-th/0606198] [inSPIRE].

[6] A. Campoleoni, D. Francia, J. Mourad and A. Sagnotti, Unconstrained higher spins of mixed symmetry II. Fermi fields, Nucl. Phys. B 828 (2010) 405 [arXiv: 0904.4447] [INSPIRE].

[7] E.D. Skvortsov, Mixed-symmetry massless fields in Minkowski space unfolded, JHEP 07 (2008) 004 [arXiv: 0801.2268] [INSPIRE].

[8] R.R. Metsaev, Massless mixed symmetry bosonic free fields in d-dimensional anti-de Sitter space-time, Phys. Lett. B 354 (1995) 78 [INSPIRE].

[9] R.R. Metsaev, Arbitrary spin massless bosonic fields in d-dimensional anti-de Sitter space, Lect. Notes Phys. 524 (1999) 331 [hep-th/9810231] [INSPIRE]. 
[10] R.R. Metsaev, Fermionic fields in the d-dimensional anti-de Sitter space-time, Phys. Lett. B 419 (1998) 49 [hep-th/9802097] [INSPIRE].

[11] C. Fronsdal, Singletons and massless, integral spin fields on de Sitter space (elementary particles in a curved space 7), Phys. Rev. D 20 (1979) 848 [InSPIRE].

[12] N. Boulanger, C. Iazeolla and P. Sundell, Unfolding mixed-symmetry fields in AdS and the BMV conjecture: I. General formalism, JHEP 07 (2009) 013 [arXiv:0812.3615] [INSPIRE].

[13] N. Boulanger, C. Iazeolla and P. Sundell, Unfolding mixed-symmetry fields in AdS and the BMV conjecture: II. Oscillator realization, JHEP 07 (2009) 014 [arXiv:0812.4438] [INSPIRE].

[14] E.D. Skvortsov, Gauge fields in (A)dS $S_{d}$ and connections of its symmetry algebra, J. Phys. A 42 (2009) 385401 [arXiv: 0904.2919] [INSPIRE].

[15] E.D. Skvortsov, Gauge fields in $(A) d S_{d}$ within the unfolded approach: algebraic aspects, JHEP 01 (2010) 106 [arXiv:0910.3334] [INSPIRE].

[16] V.E. Lopatin and M.A. Vasiliev, Free massless bosonic fields of arbitrary spin in d-dimensional de Sitter space, Mod. Phys. Lett. A 3 (1988) 257 [InSPIRE].

[17] E. Joung, J. Mourad and R. Parentani, Group theoretical approach to quantum fields in de Sitter space I. The principle series, JHEP 08 (2006) 082 [hep-th/0606119] [INSPIRE].

[18] E. Joung, J. Mourad and R. Parentani, Group theoretical approach to quantum fields in de Sitter space II. The complementary and discrete series, JHEP 09 (2007) 030 [arXiv: 0707.2907] [INSPIRE].

[19] T. Hirai, On irreducible representations of the Lorentz group of $n$-th order, Proc. Japan Acad. 38 (1962) 258.

[20] F. Schwarz, Unitary irreducible representations of the groups $\mathrm{SO}_{0}(n, 1)$, J. Math. Phys. 12 (1971) 131.

[21] V.K. Dobrev, G. Mack, V.B. Petkova, S.G. Petrova and I.T. Todorov, Harmonic analysis on the $n$-dimensional Lorentz group and its application to conformal quantum field theory, Lect. Notes Phys. 63 (1977) 1 [INSPIRE].

[22] I.T. Todorov, M.C. Mintchev and V.B. Petkova, Conformal invariance in quantum field theory, Scuola normale superiore, Classe di scienze, Pisa Italy, (1978) [InSPIRE].

[23] L. Brink, R.R. Metsaev and M.A. Vasiliev, How massless are massless fields in AdS $S_{d}$, Nucl. Phys. B 586 (2000) 183 [hep-th/0005136] [INSPIRE].

[24] K.B. Alkalaev and M. Grigoriev, Unified BRST description of AdS gauge fields, Nucl. Phys. B 835 (2010) 197 [arXiv:0910.2690] [INSPIRE].

[25] A. Campoleoni and D. Francia, Maxwell-like Lagrangians for higher spins, JHEP 03 (2013) 168 [arXiv: 1206.5877] [INSPIRE].

[26] G. Barnich and C. Troessaert, Comments on holographic current algebras and asymptotically flat four dimensional spacetimes at null infinity, JHEP 11 (2013) 003 [arXiv:1309.0794] [INSPIRE].

[27] A. Campoleoni, H.A. Gonzalez, B. Oblak and M. Riegler, Rotating higher spin partition functions and extended BMS symmetries, JHEP 04 (2016) 034 [arXiv:1512.03353] [INSPIRE]. 
[28] C. Sleight and M. Taronna, Higher-spin algebras, holography and flat space, JHEP 02 (2017) 095 [arXiv: 1609.00991] [INSPIRE].

[29] D. Ponomarev and E.D. Skvortsov, Light-front higher-spin theories in flat space, J. Phys. A 50 (2017) 095401 [arXiv: 1609.04655] [INSPIRE].

[30] E.A. Thieleker, The unitary representations of the generalized Lorentz groups, Trans. Amer. Math. Soc. 199 (1974) 327.

[31] A.M. Gavrilik and A.U. Klimyk, Analysis of the representations of the Lorentz and Euclidean groups of $n$-th order, tech. rep. ITP-75-18-E, Inst. Theor. Phys., Kiev Ukraine, (1975).

[32] A. Knapp, Representation theory of semisimple groups: an overview based on examples, Princeton Mathematical Series, Princeton University Press, Princeton U.S.A., (1986).

[33] A. Knapp, Lie groups beyond an introduction, Progress in Mathematics, Birkhäuser, Basel Switzerland, (2002).

[34] V.K. Dobrev, Intertwining operator realization of the AdS/CFT correspondence, Nucl. Phys. B 553 (1999) 559 [hep-th/9812194] [INSPIRE].

[35] A.O. Barut and R. Raczka, Theory of group representations and applications, World Scientific, Singapore, (1986) [INSPIRE].

[36] T. Hirai, The characters of irreducible representations of the Lorentz group of $n$-th order, Proc. Japan Acad. 41 (1965) 526.

[37] J. Mickelsson and J. Niederle, Contractions of representations of de Sitter groups, Commun. Math. Phys. 27 (1972) 167 [InSPIRE].

[38] A.M. Gavrilik and A.U. Klimyk, The representations of the groups $\mathrm{U}(n, 1)$ and $\mathrm{SO}(o)(n, 1)$, (1976) [INSPIRE].

[39] O.V. Shaynkman, I. Yu. Tipunin and M.A. Vasiliev, Unfolded form of conformal equations in $M$ dimensions and $o(M+2)$ modules, Rev. Math. Phys. 18 (2006) 823 [hep-th/0401086] [INSPIRE].

[40] M. Beccaria, X. Bekaert and A.A. Tseytlin, Partition function of free conformal higher spin theory, JHEP 08 (2014) 113 [arXiv: 1406. 3542] [INSPIRE].

[41] A. Higuchi, Symmetric tensor spherical harmonics on the $N$ sphere and their application to the de Sitter group $\mathrm{SO}(N, 1)$, J. Math. Phys. 28 (1987) 1553 [Erratum ibid. 43 (2002) 6385] [INSPIRE].

[42] S. Deser and R.I. Nepomechie, Gauge invariance versus masslessness in de Sitter space, Annals Phys. 154 (1984) 396 [INSPIRE].

[43] S. Deser and R.I. Nepomechie, Anomalous propagation of gauge fields in conformally flat spaces, Phys. Lett. B 132 (1983) 321 [INSPIRE].

[44] S. Deser and A. Waldron, Partial masslessness of higher spins in (A)dS, Nucl. Phys. B 607 (2001) 577 [hep-th/0103198] [INSPIRE].

[45] E. Joung and K. Mkrtchyan, Partially-massless higher-spin algebras and their finite-dimensional truncations, JHEP 01 (2016) 003 [arXiv:1508.07332] [INSPIRE].

[46] S. Gwak, J. Kim and S.-J. Rey, Massless and massive higher spins from anti-de Sitter space waveguide, JHEP 11 (2016) 024 [arXiv: 1605.06526] [INSPIRE]. 
[47] M. Günaydin, E.D. Skvortsov and T. Tran, Exceptional F(4) higher-spin theory in $A d S_{6}$ at one-loop and other tests of duality, JHEP 11 (2016) 168 [arXiv:1608.07582] [INSPIRE].

[48] C. Brust and K. Hinterbichler, Partially massless higher-spin theory, JHEP 02 (2017) 086 [arXiv: 1610.08510] [INSPIRE].

[49] C. Brust and K. Hinterbichler, Partially massless higher-spin theory II: one-loop effective actions, JHEP 01 (2017) 126 [arXiv:1610.08522] [INSPIRE].

[50] S. Deser and A. Waldron, Arbitrary spin representations in de Sitter from dS/CFT with applications to dS supergravity, Nucl. Phys. B 662 (2003) 379 [hep-th/0301068] [INSPIRE].

[51] P. de Medeiros and C. Hull, Exotic tensor gauge theory and duality, Commun. Math. Phys. 235 (2003) 255 [hep-th/0208155] [INSPIRE].

[52] E. Joung and K. Mkrtchyan, Weyl action of two-column mixed-symmetry field and its factorization around (A)dS space, JHEP 06 (2016) 135 [arXiv: 1604.05330] [INSPIRE].

[53] Yu. M. Zinoviev, First order formalism for massive mixed symmetry tensor fields in Minkowski and (A)dS spaces, hep-th/0306292 [INSPIRE].

[54] Yu. M. Zinoviev, Toward frame-like gauge invariant formulation for massive mixed symmetry bosonic fields, Nucl. Phys. B 812 (2009) 46 [arXiv:0809.3287] [INSPIRE].

[55] Yu. M. Zinoviev, Towards frame-like gauge invariant formulation for massive mixed symmetry bosonic fields II. General Young tableau with two rows, Nucl. Phys. B 826 (2010) 490 [arXiv: 0907.2140] [INSPIRE].

[56] Yu. M. Zinoviev, Gravitational cubic interactions for a massive mixed symmetry gauge field, Class. Quant. Grav. 29 (2012) 015013 [arXiv:1107.3222] [INSPIRE].

[57] M. Flato and C. Fronsdal, One massless particle equals two Dirac singletons: elementary particles in a curved space. 6, Lett. Math. Phys. 2 (1978) 421 [INSPIRE].

[58] E. Sezgin and P. Sundell, Massless higher spins and holography, Nucl. Phys. B 644 (2002) 303 [Erratum ibid. B 660 (2003) 403] [hep-th/0205131] [INSPIRE].

[59] I.R. Klebanov and A.M. Polyakov, AdS dual of the critical $O(N)$ vector model, Phys. Lett. B 550 (2002) 213 [hep-th/0210114] [INSPIRE].

[60] D. Anninos, T. Hartman and A. Strominger, Higher spin realization of the $d S / C F T$ correspondence, Class. Quant. Grav. 34 (2017) 015009 [arXiv:1108.5735] [INSPIRE].

[61] C. Iazeolla and P. Sundell, A fiber approach to harmonic analysis of unfolded higher-spin field equations, JHEP 10 (2008) 022 [arXiv:0806.1942] [INSPIRE].

[62] E. Sezgin and P. Sundell, Supersymmetric higher spin theories, J. Phys. A 46 (2013) 214022 [arXiv:1208.6019] [INSPIRE].

[63] E. Joung and K. Mkrtchyan, Notes on higher-spin algebras: minimal representations and structure constants, JHEP 05 (2014) 103 [arXiv:1401.7977] [INSPIRE].

[64] R. Raczka, N. Limić and J. Niederle, Discrete degenerate representations of noncompact rotation groups I, J. Math. Phys. 7 (1966) 1861.

[65] P. Breitenlohner and D.Z. Freedman, Positive energy in anti-de Sitter backgrounds and gauged extended supergravity, Phys. Lett. B 115 (1982) 197 [INSPIRE].

[66] P. Breitenlohner and D.Z. Freedman, Stability in gauged extended supergravity, Annals Phys. 144 (1982) 249 [INSPIRE]. 
[67] K. Skenderis, Lecture notes on holographic renormalization, Class. Quant. Grav. 19 (2002) 5849 [hep-th/0209067] [INSPIRE].

[68] X. Bekaert and M. Grigoriev, Higher order singletons, partially massless fields and their boundary values in the ambient approach, Nucl. Phys. B 876 (2013) 667 [arXiv:1305.0162] [INSPIRE].

[69] F.A. Dolan, Character formulae and partition functions in higher dimensional conformal field theory, J. Math. Phys. 47 (2006) 062303 [hep-th/0508031] [INSPIRE].

[70] J. Lepowsky, A generalization of the Bernstein-Gelfand-Gelfand resolution, J. Alg. 49 (1977) 496.

[71] B. Oblak, BMS particles in three dimensions, arXiv:1610.08526 [INSPIRE].

[72] W. Fulton and J. Harris, Representation theory: a first course, Grad. Texts Math. 129, Springer New York U.S.A., (1991).

[73] T. Hirai, On infinitesimal operators of irreducible representations of the Lorentz group of n-th order, Proc. Japan Acad. 38 (1962) 83. 